\title{
Adaptive Observers and Parameter Estimation for a Class of Systems Nonlinear in the Parameters
}

\author{
Ivan Y. Tyukin ${ }^{a}$ Erik Steur ${ }^{b}$ Henk Nijmeijer ${ }^{c}$ Cees van Leeuwen ${ }^{b}$ \\ ${ }^{a}$ Dept. of Mathematics, University of Leicester, Leicester, LE1 7RH, UK (Tel: +44-116-252-5106; I.Tyukin@le.ac.uk) \\ ${ }^{\mathrm{b}}$ Laboratory for Perceptual Dynamics, KU Leuven, Tiensestraat 102,3000 Leuven, Belgium (erik.steur@ppw.kuleuven.be, \\ cees.vanleeuwen@ppw.kuleuven.be) \\ ${ }^{\mathrm{c}}$ Dept. of Mechanical Engineering, Eindhoven University of Technology, P.O. Box 5135600 MB, Eindhoven, The \\ Netherlands,(h.nijmeijer@tue.nl)
}

\begin{abstract}
We consider the problem of asymptotic reconstruction of the state and parameter values in systems of ordinary differential equations. A solution to this problem is proposed for a class of systems of which the unknowns are allowed to be nonlinearly parameterized functions of state and time. Reconstruction of state and parameter values is based on the concepts of weakly attracting sets and non-uniform convergence and is subjected to persistency of excitation conditions. In the absence of nonlinear parametrization the resulting observers reduce to standard estimation schemes. In this respect, the proposed method constitutes a generalization of the conventional canonical adaptive observer design.
\end{abstract}

Key words: Adaptive observers, nonlinear parametrization, weakly attracting sets

\section{Introduction}

We consider observer-based methods for state and parameter estimation in nonlinear dynamical systems. These methods are effective as long as the original system has, or can be transformed into, one of the canonical adaptive observer forms [5], [25], [6]. Their common characteristic is linearity in the unknown parameters. For this class of systems, subject to persistency of excitation conditions, reconstruction of state and parameter vectors can be achieved exponentially fast.

There are systems, however, in which the unknown parameters enter the model nonlinearly. These systems constitute a remarkably wide class including models in chemical kinetics [13], [4], biology and neuroscience [18]. Whereas the problem of state estimation can be solved for a large class of nonlinearly parameterized systems [28], observer-based parameter reconstruction is often confined to systems with monotone [39], [22] or one-toone parameterizations [14], [15], [16], [12].

\footnotetext{
^ This paper was not presented at any IFAC meeting. Cees van Leeuwen is supported by an Odysseus grant from the Flemish Science Organization FWO. Corresponding author
} I. Yu. Tyukin. Tel. +44-116-2525106.
Several authors have recently advanced strategies for overcoming these limitations. In [19], combining interval analysis with multiple shooting methods is proposed to tackle the state and parameter reconstruction problem. Another interesting approach is presented in [1]: the original continuous-time model is replaced with a discretetime approximation. Measured variables are then considered as known functions of unknown parameters and initial conditions, of which the estimates can be found by off-line nonlinear optimization routines (see also [36], [2] where optimization techniques with moving horizon are discussed). These approaches offer obvious advantages, e.g. the availability of a vast library of numerical methods for solving general nonlinear optimization problems. Nevertheless, these methods run into restrictions too. Exhaustive search for a global minimum may become intractable for dimensions higher than 1 or 2 . On the other hand, if conventional polynomial-complexity algorithms are used then the possibility arises that the algorithm will converge to a local minimum.

In this paper we explore further possibilities of developing adaptive observers for systems which are both linearly and nonlinearly parameterized. The parametrization is not required to be invertible or monotone. Our approach combines the advantages of the existing schemes, 
in being capable of ensuring exponentially fast convergence with the flexibility of explorative behavior, a behavior inherent to algorithms for solving genuine nonlinear optimization problems. Inference of the values of state and a part of the parameter vector of these systems is achieved by employing exponentially fast converging estimators. Estimation of the values of the remaining parameters is based on an explorative search procedure. Since exploration is restricted to a subset of the unknown parameters, the proposed strategy reduces the overall computational costs, as compared to when full-scale search-based optimization had been invoked.

The resulting observer can be imagined as a system comprising of an exponentially stable part coupled with an explorative one. Systems of this type have previously been used in adaptive control [34], [17], [29], [30]. Here we demonstrate that these classical ideas can be applied to the problem of adaptive observer design for systems which are nonlinearly dependent on parameters. We show that, subject to a condition of persistent excitation, it is possible to reconstruct state and parameters of a reasonably broad subclass of these systems.

The paper is organized as follows. Notational agreements are introduced in Section 2. Section 3 provides the formal statement of the problem, Sections 4, 5 contain main results of the article, Section 6 discusses possible generalizations, Section 7 contains illustrative examples, and Section 8 concludes the paper. Proofs of auxiliary results are presented in the Appendix.

\section{Notation}

The following notational conventions are used throughout the paper:

- $\mathbb{R}$ denotes the set of real numbers, $\mathbb{R}_{>a}=\{x \in \mathbb{R} \mid x>$ $a\}$, and $\mathbb{R}_{\geq a}=\{x \in \mathbb{R} \mid x \geq a\}$;

- $\mathbb{Z}$ denotes the set of integers, and $\mathbb{N}$ stands for the set of positive integers;

- the Euclidean norm of $\mathbf{x} \in \mathbb{R}^{n}$ is denoted by $\|\mathbf{x}\|$, $\|\mathbf{x}\|^{2}=\mathbf{x}^{T} \mathbf{x}$, where ${ }^{T}$ stands for transposition;

- the space of $n \times n$ matrices with real entries is denoted by $\mathbb{R}^{n \times n}$; let $\mathbf{P} \in \mathbb{R}^{n \times n}$, then $\mathbf{P}>0(\mathbf{P} \geq 0)$ indicates that $\mathbf{P}$ is symmetric and positive (semi-)definite; $\mathbf{I}_{n}$ denotes the $n \times n$ identity matrix.

- by $L_{\infty}^{n}\left[t_{0}, T\right], t_{0} \in \mathbb{R}, T \in \mathbb{R}, T \geq t_{0}$ we denote the space of all functions $\mathbf{f}:\left[t_{0}, \bar{T}\right] \rightarrow \mathbb{R}^{n}$ such that $\|\mathbf{f}\|_{\infty,\left[t_{0}, T\right]}=\operatorname{ess} \sup \left\{\|\mathbf{f}(t)\|, t \in\left[t_{0}, T\right]\right\}<\infty$; $\|\mathbf{f}\|_{\infty,\left[t_{0}, T\right]}$ stands for the $L_{\infty}^{n}\left[t_{0}, T\right]$ norm of $\mathbf{f}(t)$; if the function $\mathbf{f}$ is defined on a set larger than $\left[t_{0}, T\right]$ then notation $\|\mathbf{f}\|_{\infty,\left[t_{0}, T\right]}$ applies to the restriction of f on $\left[t_{0}, T\right]$

- $\mathcal{C}^{r}$ denotes the space of continuous functions that are at least $r$ times differentiable;

- Let $\mathcal{A}$ be a subset of $\mathbb{R}^{n}$, then for all $\mathbf{x} \in \mathbb{R}^{n}$, we define $\operatorname{dist}(\mathcal{A}, \mathbf{x})=\inf _{\mathbf{q} \in \mathcal{A}}\|\mathbf{x}-\mathbf{q}\| ;$
- A solution of $\dot{\mathbf{x}}=\mathbf{f}(t, \mathbf{x}, \boldsymbol{\theta}, u(t)), \mathbf{f}: \mathbb{R} \times \mathbb{R}^{n} \times \mathbb{R}^{m} \times$ $\mathbb{R} \rightarrow \mathbb{R}^{n}, \boldsymbol{\theta} \in \mathbb{R}^{m}, u: \mathbb{R} \rightarrow \mathbb{R}$ passing through $\mathbf{x}_{0} \in$ $\mathbb{R}^{n}$ at $t=t_{0}$ is denoted by $\mathbf{x}\left(t, t_{0}, \mathbf{x}_{0}, \boldsymbol{\theta},[u]\right)$. In cases when $u$ and/or $\boldsymbol{\theta}, \mathbf{x}_{0}, t_{0}$ are clearly determined by the context a more compact notation, $\mathbf{x}\left(t, t_{0}, \mathbf{x}_{0}, \boldsymbol{\theta}\right)$ (or $\mathbf{x}\left(t, t_{0}, \mathbf{x}_{0}\right), \mathbf{x}(t), \mathbf{x}$ respectively), is used.

- The symbol $\mathcal{K}$ denotes the class of all strictly increasing continuous functions $\kappa: \mathbb{R}_{\geq 0} \rightarrow \mathbb{R}_{\geq 0}$ such that $\kappa(0)=0$; the symbol $\mathcal{K}_{\infty}$ denotes the class of all functions $\kappa \in \mathcal{K}$ such that $\lim _{s \rightarrow \infty} \kappa(s)=\infty$.

- Let $\epsilon \in \mathbb{R}_{\geq 0}$, then $\|\mathbf{x}\|_{\epsilon}$ stands for: $\|\mathbf{x}\|-\epsilon$ if $\|\mathbf{x}\|>\epsilon$, and 0 otherwise.

- Finally, for $\boldsymbol{\lambda} \in \mathbb{R}^{p}$ and $\boldsymbol{\theta} \in \mathbb{R}^{m}$, the notation $(\boldsymbol{\lambda}, \boldsymbol{\theta})$ stands for $\operatorname{col}\left(\lambda_{1}, \ldots, \lambda_{p}, \theta_{1}, \ldots, \theta_{m}\right)$.

\section{Preliminaries and Problem formulation}

\subsection{Adaptive observer canonical form}

Throughout the paper we will focus exclusively on the class of systems that are forward-complete:

Definition 1 Let $\mathcal{L}_{u}\left[t_{0}, \infty\right]$ be a subspace of $L_{\infty}\left[t_{0}, \infty\right]$. A single-input single-output system described by $\dot{\mathbf{x}}=$ $\mathbf{f}(t, \mathbf{x}, u(t)), \mathbf{f}: \mathbb{R} \times \mathbb{R}^{n} \times \mathbb{R} \rightarrow \mathbb{R}^{n}, y=\mathbf{h}(t, \mathbf{x}), \mathbf{h}:$ $\mathbb{R} \times \mathbb{R}^{n} \rightarrow \mathbb{R}, u \in \mathcal{L}_{u}\left[t_{0}, \infty\right]$, where $u$ is the input, and $y$ is the output, is called forward-complete (with respect to $\left.\mathcal{L}_{u}\right)$ iff for any $t_{0} \in \mathbb{R}, \mathbf{x}_{0} \in \mathbb{R}^{n}$, and $u \in \mathcal{L}_{u}\left[t_{0}, \infty\right]$ the solution $\mathbf{x}\left(t, t_{0}, \mathbf{x}_{0},[u]\right)$ exists and is defined for all $t \geq t_{0}$.

Let $\mathcal{L}_{u}\left[t_{0}, \infty\right]=L_{\infty}\left[t_{0}, \infty\right] \cap \mathcal{C}^{0}\left[t_{0}, \infty\right]$, and consider a forward-complete single-input single-output system; let $\mathbf{x} \in \mathbb{R}^{n}$ be its state, $y: \mathbb{R} \rightarrow \mathbb{R}$ be the measured output, and $u: \mathbb{R} \rightarrow \mathbb{R}, u \in \mathcal{L}_{u}$ be the input. We recall that a system is in the adaptive observer canonical form if it is governed by the following set of equations

$$
\begin{aligned}
& \dot{\mathbf{x}}=\mathbf{A} \mathbf{x}+\mathbf{B} \phi^{T}(t, y) \boldsymbol{\theta}+\mathbf{g}(t, y, u(t)) \\
& y=\mathbf{C}^{T} \mathbf{x}, \mathbf{x}\left(t_{0}\right)=\mathbf{x}_{0}, \mathbf{x}_{0} \in \mathbb{R}^{n}
\end{aligned}
$$

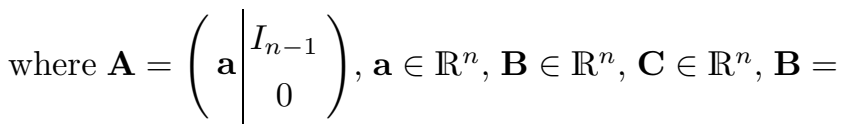
$\operatorname{col}\left(1, b_{1}, \ldots, b_{n-1}\right), \mathbf{C}=\operatorname{col}(1,0, \ldots, 0)$, the functions $\phi: \mathbb{R} \times \mathbb{R} \rightarrow \mathbb{R}^{m}, \mathbf{g}: \mathbb{R} \times \mathbb{R} \times \mathbb{R} \rightarrow \mathbb{R}^{n}, \boldsymbol{\phi}, \mathbf{g} \in \mathcal{C}^{0}$ are known, and $\boldsymbol{\theta} \in \mathbb{R}^{m}$ is the vector of the unknown parameters. The triplet $\mathbf{A}, \mathbf{B}, \mathbf{C}$ is supposed to satisfy

$$
\left\{\begin{array}{l}
\mathbf{P}\left(\mathbf{A}+\boldsymbol{\ell} \mathbf{C}^{T}\right)+\left(\mathbf{A}+\boldsymbol{\ell} \mathbf{C}^{T}\right)^{T} \mathbf{P} \leq-\mathbf{Q} \\
\mathbf{P B}=\mathbf{C} .
\end{array}\right.
$$

for some $\boldsymbol{\ell} \in \mathbb{R}^{n}$ and $\mathbf{P}>0, \mathbf{Q}>0$. Although condition (2) may appear restrictive, it has been shown in [26] that subject to the very natural constraint that the pair $\mathbf{A}, \mathbf{C}$ is observable, there is a time-varying parameterdependent coordinate transformation such that in new 
coordinates the system is still of the form (1) and satisfies condition (2). If requirement (2) holds then the system

$$
\begin{aligned}
\dot{\hat{\mathbf{x}}}= & \mathbf{A} \hat{\mathbf{x}}+\boldsymbol{\ell}\left(\mathbf{C}^{T} \hat{\mathbf{x}}-y(t)\right)+\mathbf{B} \boldsymbol{\phi}^{T}(t, y(t)) \hat{\boldsymbol{\theta}} \\
& +\mathbf{g}(t, y(t), u(t)) \\
\dot{\hat{\boldsymbol{\theta}}}= & -\gamma\left(\mathbf{C}^{T} \hat{\mathbf{x}}-y(t)\right) \boldsymbol{\phi}(t, y(t)), \gamma \in \mathbb{R}_{>0},
\end{aligned}
$$

where $\hat{\mathbf{x}}(t) \in \mathbb{R}^{n}, \hat{\boldsymbol{\theta}}(t) \in \mathbb{R}^{m}$, is an adaptive observer for (1) (cf. [25], [5]) provided that the restriction of $\phi(\cdot, y(\cdot))$ on $\mathbb{R}_{\geq t_{0}}$ is persistently exciting:

Definition 2 A function $\boldsymbol{\beta}: \mathbb{R}_{\geq t_{0}} \rightarrow \mathbb{R}^{m}$ is said to be persistently exciting if there exist $L, \mu \in \mathbb{R}_{>0}$ :

$$
\int_{t}^{t+L} \boldsymbol{\beta}(\tau) \boldsymbol{\beta}^{T}(\tau) \mathrm{d} \tau \geq \mu \mathbf{I}_{m}, \forall t \geq t_{0}
$$

The fact that (3) is an adaptive observer for (1) is based on a well-known result on the exponential stability of the following class of linear time-varying system: 1

$$
\dot{\mathbf{e}}=\mathbf{A}(t) \mathbf{e}, \mathbf{A}(t)=\left(\begin{array}{cc}
\mathbf{A}+\boldsymbol{\ell} \mathbf{C}^{T} & \mathbf{B} \boldsymbol{\beta}^{T}(t) \\
-\boldsymbol{\beta}(t) \mathbf{C}^{T} & 0
\end{array}\right)
$$

The result is provided in Theorem 3 below (see e.g. [23] for a proof).

Theorem 3 Consider system (5). Suppose that condition (2) holds for certain $\boldsymbol{\ell} \in \mathbb{R}^{n}, \mathbf{P}>0, \mathbf{Q}>0$, the function $\boldsymbol{\beta}(t)$ is persistently exciting, and

$$
\exists M \in \mathbb{R}_{>0}: \quad \max \{\|\boldsymbol{\beta}(t)\|,\|\dot{\boldsymbol{\beta}}(t)\|\} \leq M \forall t \geq t_{0} .
$$

Let $\Phi\left(t, t_{0}\right), \Phi\left(t_{0}, t_{0}\right)=I_{n+m}$, be the fundamental solution matrix of (5). Then there exist $\rho, D \in \mathbb{R}_{>0}$ such that $\left\|\Phi\left(t_{2}, t_{1}\right) \mathbf{p}\right\| \leq D e^{-\rho\left(t_{2}-t_{1}\right)}\|\mathbf{p}\|$ for all $t_{2} \geq t_{1} \geq t_{0}$ and $\mathbf{p} \in \mathbb{R}^{n+m}$.

The parameters $\rho$ and $D$ can be expressed explicitly as functions of $M, \mu, L$, and $\mathbf{A}, \mathbf{B}, \mathbf{C}, \boldsymbol{\ell}[23]$. By letting $\mathbf{e}=$ $\operatorname{col}(\hat{\mathbf{x}}-\mathbf{x}, \hat{\boldsymbol{\theta}}-\boldsymbol{\theta})$ and taking (1), (3) into account one can confirm that the system-observer equations are of form (5). Thus, subject to persistency of excitation of the restriction of $\boldsymbol{\phi}^{T}(\cdot, y(\cdot))$ on $\mathbb{R}_{\geq t_{0}}, \lim _{t \rightarrow \infty} \hat{\mathbf{x}}\left(t, t_{0}, \hat{\mathbf{x}}_{0}, \hat{\boldsymbol{\theta}}_{0}\right)-$ $\mathbf{x}\left(t, t_{0}, \mathbf{x}_{0}, \boldsymbol{\theta}\right)=0, \lim _{t \rightarrow \infty} \hat{\boldsymbol{\theta}}\left(t, t_{0}, \hat{\mathbf{x}}_{0}, \hat{\boldsymbol{\theta}}_{0}\right)=\boldsymbol{\theta}$ along the solutions of (1), (3), and the convergence is exponential. The problem, however, is that if some parameters enter the equations nonlinearly then this creates an obstacle for the explicit use of Theorem 3 and, consequently, observer (3). In the next sections we present and analyze a class of systems nonlinear in the parameters which can be thought of as an immediate generalization of (1).

\footnotetext{
1 In the context of adaptive observer design for (1) the func-
} tion $\boldsymbol{\beta}$ in (5) is defined as $\boldsymbol{\beta}(t)=\boldsymbol{\phi}(t, y(t)), t \geq t_{0}$

\subsection{Systems considered in this article}

We begin with the following class of forward-complete single-input-single-output nonlinear systems:

$$
\begin{aligned}
\dot{\mathbf{x}} & =\mathbf{A} \mathbf{x}+\mathbf{B} \varphi^{T}(t, \boldsymbol{\lambda}, y) \boldsymbol{\theta}+\mathbf{g}(t, \boldsymbol{\lambda}, y, u(t))+\boldsymbol{\xi}(t), \\
y & =\mathbf{C}^{T} \mathbf{x}, \mathbf{x}\left(t_{0}\right)=\mathbf{x}_{0}, \mathbf{x}_{0} \in \mathbb{R}^{n},
\end{aligned}
$$

where $\mathbf{A} \in \mathbb{R}^{n \times n}$, and $\mathbf{B}, \mathbf{C} \in \mathbb{R}^{n}$ are defined as in (1); $\boldsymbol{\varphi}: \mathbb{R} \times \mathbb{R}^{p} \times \mathbb{R} \rightarrow \mathbb{R}^{m}, \mathbf{g}: \mathbb{R} \times \mathbb{R}^{p} \times \mathbb{R} \times \mathbb{R} \rightarrow \mathbb{R}^{n}$, are known continuous functions, $\boldsymbol{\lambda}=\operatorname{col}\left(\lambda_{1}, \ldots, \lambda_{p}\right) \in \mathbb{R}^{p}$, $\boldsymbol{\theta}=\operatorname{col}\left(\theta_{1}, \ldots, \theta_{m}\right) \in \mathbb{R}^{m}$ are unknown parameters, and $u \in \mathcal{L}_{u} \cap \mathcal{C}^{1}$, is the input. We assume that the values of $\boldsymbol{\lambda}, \boldsymbol{\theta}$ belong to the hypercubes $\Omega_{\lambda} \subset \mathbb{R}^{p}, \Omega_{\theta} \subset \mathbb{R}^{m}$ with known bounds: $\theta_{i} \in\left[\theta_{i, \min }, \theta_{i, \max }\right], \lambda_{j} \in\left[\lambda_{j, \min }, \lambda_{j, \max }\right]$, and that $y(t) \in \mathcal{D}_{y}, u(t) \in \mathcal{D}_{u}, \mathcal{D}_{y}, \mathcal{D}_{u} \subset \mathbb{R}$ for $t \geq t_{0}$.

In $(7), \mathbf{x}=\operatorname{col}\left(x_{1}, x_{2}, \ldots, x_{n}\right) \in \mathbb{R}^{n}$ is the state vector, $y$ is the measured output, the input $u$ is a known function, and $\boldsymbol{\xi} \in \mathcal{C}^{0}: \mathbb{R} \rightarrow \mathbb{R}^{n}$ is an unknown yet bounded continuous function:

$$
\exists \Delta_{\xi} \in \mathbb{R}_{\geq 0}: \quad\|\boldsymbol{\xi}(t)\| \leq \Delta_{\xi} \forall t
$$

representing some unmodeled dynamics (e.g. noise). The system's state $\mathbf{x}$ is not measured; only the values of the input $u(t)$ and the output $y(t)=x_{1}(t), t \geq t_{0}$ in $(7)$ are accessible over any time interval $\left[t_{0}, t\right]$ that belongs to the history of the system.

For the time being we suppose that matrix $\mathbf{A}$ and vectors B, C in (7) satisfy Assumption 3.1 below.

Assumption 3.1 The triple A, B, C is known, and there exist (and are known) a vector $\boldsymbol{\ell}$ and matrices $\mathbf{P}, \mathbf{Q}>0$ such that condition (2) holds.

Note that Assumption 3.1 implies that the vector $\mathbf{B}=$ $\operatorname{col}\left(1, b_{1}, \ldots, b_{n-1}\right)$ in $(7)$ is such that the polynomial $s^{n-1}+b_{1} s^{n-2}+\cdots+b_{n-1}$ is Hurwitz. At first, Assumption 3.1 may seem restrictive. In Section 6 we lift this restriction by showing that the results presented for (7) can be generalized to systems

$$
\begin{aligned}
& \dot{\mathbf{x}}=\mathbf{A x}+\boldsymbol{\Psi}(t, \boldsymbol{\lambda}, y) \boldsymbol{\theta}+\mathbf{g}(t, \boldsymbol{\lambda}, y, u(t))+\boldsymbol{\xi}(t), \\
& y=\mathbf{C}^{T} \mathbf{x}, \mathbf{C}=\operatorname{col}(1,0, \ldots, 0),
\end{aligned}
$$

in which the matrix $\mathbf{A} \in \mathbb{R}^{n \times n}$ may be unknown but it is known that the pair $\mathbf{A}, \mathbf{C}$ is observable, the function $\boldsymbol{\Psi}: \mathbb{R} \times \mathbb{R}^{p} \times \mathbb{R} \rightarrow \mathbb{R}^{n \times m}, \boldsymbol{\Psi} \in \mathcal{C}^{1}$, is Lipschitz in $\boldsymbol{\lambda}$, and $\mathbf{g}(\cdot, \boldsymbol{\lambda}, y(\cdot), u(\cdot)), \dot{\mathrm{g}}(\cdot, \boldsymbol{\lambda}, y(\cdot), u(\cdot)), \mathbf{\Psi}(\cdot, \boldsymbol{\lambda}, y(\cdot))$, $\dot{\boldsymbol{\Psi}}(\cdot, \boldsymbol{\lambda}, y(\cdot))$ are bounded for all $\boldsymbol{\lambda} \in \Omega_{\lambda}$ on $\left[t_{0}, \infty\right)$.

With regards to the functions $\varphi$ and $\mathbf{g}$ in (7) the following additional technical assumptions are made: 
Table 1

Examples of physical systems in the form of (7), (9)

\begin{tabular}{|c|c|c|}
\hline tem & Model & Possible parametrization: $\boldsymbol{\theta}, \boldsymbol{\lambda}, \mathbf{A}, \mathbf{B}, \boldsymbol{\varphi}, \boldsymbol{\Psi}, \mathbf{g}$ \\
\hline $\begin{array}{l}\text { 1. React } \\
\text { namics ir } \\
\text { tor }[35]\end{array}$ & $\begin{array}{l}\dot{x}=\frac{F}{V}\left(x_{0}-x\right)-k_{\mathrm{ref}} e^{-\frac{E}{R}\left(\frac{1}{T(t)}-\frac{1}{T_{\mathrm{ref}}}\right)} x, \\
y=x\end{array}$ & $\begin{array}{l}\text { System }(7): \boldsymbol{\theta}=\operatorname{col}\left(\frac{F}{V} x_{0},-\frac{F}{V},-k_{\mathrm{ref}} e^{\frac{E}{R T_{\mathrm{ref}}}}\right), \lambda=-\frac{E}{R}, \\
\mathbf{A}=0, \mathbf{B}=1, \boldsymbol{\varphi}(t, \lambda, y)=\operatorname{col}\left(1, y, e^{\lambda \frac{1}{T(t)}} y\right), g=0\end{array}$ \\
\hline $\begin{array}{l}\text { 2. Mag } \\
\text { ings }[2\end{array}$ & $\begin{array}{l}\ddot{x}+J^{-1} d(t)=b\left(\frac{q_{2}^{2}\left(I_{2}, a, x\right)}{(x+a)^{2}}-\frac{q_{1}^{2}\left(I_{1}, a, x\right)}{(x-a)^{2}}\right) \\
y=x,|x|<a-\varepsilon, a, \varepsilon \in \mathbb{R}_{>0}, \\
d, \dot{d} \text { are bounded }\end{array}$ & $\begin{array}{l}\text { System }(7): \boldsymbol{\theta}=\operatorname{col}\left(-J^{-1}, b\right), \mathbf{A}=\left(\begin{array}{ll}0 & 1 \\
0 & 0\end{array}\right), \mathbf{B}=\left(\begin{array}{l}0 \\
1\end{array}\right), \\
\lambda=a, \varphi(t, \lambda, y)=\operatorname{col}\left(d(t), \frac{q_{2}^{2}\left(I_{2}, \lambda, y\right)}{(y+\lambda)^{2}}-\frac{q_{1}^{2}\left(I_{1}, \lambda, y\right)}{(y-\lambda)^{2}}\right), g=0\end{array}$ \\
\hline $\begin{array}{l}\text { 3. Act } \\
\text { tials in }\end{array}$ & $\begin{array}{l}\dot{x}_{1}=-\frac{x_{1}}{\tau_{m}}+\frac{A_{f}}{\tau_{m}} \tanh \left(\frac{\sigma_{f}}{A_{f}} x_{1}\right)-\frac{x_{2}}{\tau_{m}} \\
\dot{x}_{2}=-\frac{x_{2}}{\tau_{s}}+\frac{\sigma_{s}}{\tau_{s}} x_{1} \\
y=x_{1}\end{array}$ & $\begin{array}{l}\text { System }(9): \boldsymbol{\theta}=\operatorname{col}\left(-\frac{1}{\tau_{m}}, \frac{A_{f}}{\tau_{m}}, \frac{\sigma_{s}}{\tau_{s}}\right), \lambda=\frac{\sigma_{f}}{A_{f}}, \mathbf{A}= \\
\left(\begin{array}{l}0-\frac{1}{\tau_{m}} \\
0-\frac{1}{\tau_{s}}\end{array}\right), \boldsymbol{\Psi}(t, \lambda, y)=\left(\begin{array}{ccc}y \tanh (\lambda y) & 0 \\
0 & 0 & y\end{array}\right), \mathbf{g}=0\end{array}$ \\
\hline
\end{tabular}

Assumption 3.2 The functions $\boldsymbol{\varphi}(\cdot, \boldsymbol{\lambda}, \cdot), \mathbf{g}(\cdot, \boldsymbol{\lambda}, \cdot, \cdot)$ in (7) are bounded and differentiable in $\mathbb{R}_{\geq t_{0}} \times \mathcal{D}_{y}$ and $\mathbb{R}_{\geq t_{0}} \times \mathcal{D}_{y} \times \mathcal{D}_{u}$ respectively, and Lipschitz in $\boldsymbol{\lambda}$. That is, there exist $D_{\varphi}, D_{g}, B_{\varphi}, B_{g} \in \mathbb{R}_{\geq 0}$ such that for all $t \in \mathbb{R}_{\geq t_{0}}, y \in \mathcal{D}_{y}, u \in \mathcal{D}_{u}, \lambda^{\prime}, \lambda^{\prime \prime} \in \Omega_{\lambda}$

$$
\begin{gathered}
\left\|\boldsymbol{\varphi}\left(t, \boldsymbol{\lambda}^{\prime}, y\right)-\varphi\left(t, \boldsymbol{\lambda}^{\prime \prime}, y\right)\right\| \leq D_{\varphi}\left\|\boldsymbol{\lambda}^{\prime}-\boldsymbol{\lambda}^{\prime \prime}\right\|, \\
\left\|\mathbf{g}\left(t, \boldsymbol{\lambda}^{\prime}, y, u\right)-\mathbf{g}\left(t, \boldsymbol{\lambda}^{\prime \prime}, y, u\right)\right\| \leq D_{g}\left\|\boldsymbol{\lambda}^{\prime}-\boldsymbol{\lambda}^{\prime \prime}\right\|, \\
\|\boldsymbol{\varphi}(t, \boldsymbol{\lambda}, y)\| \leq B_{\varphi},\|\mathbf{g}(t, \boldsymbol{\lambda}, y, u)\| \leq B_{g} .
\end{gathered}
$$

Furthermore, there exist $M_{\varphi}, M_{g} \in \mathbb{R}_{\geq 0}$ such that

$$
\begin{aligned}
& \left|\frac{\partial \varphi(t, \boldsymbol{\lambda}, y)}{\partial y} \dot{y}+\frac{\partial \varphi(t, \boldsymbol{\lambda}, y)}{\partial t}\right| \leq M_{\varphi}, \\
& \left|\frac{\partial \mathbf{g}(t, \boldsymbol{\lambda}, y, u)}{\partial y} \dot{y}+\frac{\partial \mathbf{g}(t, \boldsymbol{\lambda}, y, u)}{\partial u} \dot{u}+\frac{\partial \mathbf{g}(t, \boldsymbol{\lambda}, y, u)}{\partial t}\right| \leq M_{g}
\end{aligned}
$$

for all $\boldsymbol{\lambda} \in \Omega_{\lambda}, t \geq t_{0}$ along the solutions of ( 7$)$.

Conditions (10), (11) often hold naturally in the context of modeling and identification; they may, however, impose limitations in the framework of controller design. As for condition (12), the first inequality is a version of (6) that is essential for uniform exponential convergence of solutions to the origin of (5) [23]. The second inequality in (12) is a technical condition. Although this latter condition may look somewhat restrictive, it may be relaxed if $\mathbf{g}(t, \boldsymbol{\lambda}, y, u(t))$ is expressed as $\mathbf{g}(t, \boldsymbol{\lambda}, y, u(t))=$ $\mathbf{g}_{1}(t, y, u(t))+\mathbf{g}_{2}(t, \boldsymbol{\lambda}, y, u(t))$. In this case we would require that (11), (12) hold for $\mathbf{g}_{2}$.

A non-exhaustive list of systems that are relevant in engineering applications and are governed by (7) or (9) includes bio-/ chemical reactors $[7,35]$, nonlinear saturated magnetic circuits [32], magnetic bearings [21], tireroad interaction, and dynamics of live cells [37]. A few examples from this list are provided in Table 1. The first model, if described by (7), trivially satisfies Assumption 3.1; it also satisfies Assumption 3.2 if $T$ is bounded, differentiable, separated away from zero, and $y, \dot{T}$ are bounded. In the second model the pair $\mathbf{A}, \mathbf{C}$ is observable, and $\boldsymbol{\varphi}, \dot{\varphi}$ are bounded if $y$, magnetic fluxes, expressed by $q_{1}, q_{2}$, and $\dot{q}_{1}, \dot{q}_{2}$ are bounded. This is achievable via external controls [21], at least for small parametric mismatches and $d$. In the third model the pair $\mathbf{A}, \mathbf{C}$ is observable, and boundedness of $y, \dot{y}, \boldsymbol{\Psi}, \dot{\boldsymbol{\Psi}}$ is consistent with the physics of the system.

\subsection{Problem formulation}

Before we proceed with a formal problem statement, several points related to parametrization of $(7),(9)$ need to be discussed. First, note that different definitions of systems (7), (9) may correspond the same physical model. For example, functions $\boldsymbol{\varphi}$ and $g$ and parameters $\boldsymbol{\theta}, \boldsymbol{\lambda}$ for the first model in Table 1 may also be defined as:

$$
\begin{aligned}
& \boldsymbol{\varphi}(t, \boldsymbol{\lambda}, y)=\operatorname{col}\left(1, y, e^{\lambda_{1} \frac{1}{T(t)}+\lambda_{2}} y\right), g=0 \\
& \boldsymbol{\theta}=\operatorname{col}\left(\frac{F}{V} x_{0},-\frac{F}{V},-k_{\text {ref }}\right), \boldsymbol{\lambda}=\operatorname{col}\left(-\frac{E}{R}, \frac{E}{R T_{\text {ref }}}\right), \text { or } \\
& \boldsymbol{\varphi}(t, \boldsymbol{\lambda}, y)=\operatorname{col}(1, y), g(t, \boldsymbol{\lambda}, y, u(t))=-e^{\lambda_{1} \frac{1}{T(t)}+\lambda_{2}} y,
\end{aligned}
$$

with $\boldsymbol{\theta}=\operatorname{col}\left(\frac{F}{V} x_{0},-\frac{F}{V}\right), \boldsymbol{\lambda}=\operatorname{col}\left(-\frac{\ln \left(k_{\mathrm{ref}}\right) E}{R}, \frac{\ln \left(k_{\mathrm{ref}}\right) E}{R T_{\mathrm{ref}}}\right)$. It is clear that if parametrization (13) is chosen then identical outputs $y(t)$ will be observed for infinitely many combinations of parameters $\theta_{3}, \lambda_{2}$. Models of this type are referred to as unidentifiable [11] (see also [9], [10]). Dealing with unidentifiable models imposes technical difficulties. We will therefore assume that parametrizations which are obviously unidentifiable are avoided, if possible. As for the remaining alternative parametrizations, we assume that preference is given to those in which the dimension of $\boldsymbol{\lambda}$ is minimal, i.e. the parametrization in the first row in Table 1 is preferable to $(14)$.

Second, as far as identifiability is concerned, inferring true values of $\boldsymbol{\theta}, \boldsymbol{\lambda}$ from output observations, $y(t)$, is not 
always possible, even if the system is linearly parameterized and no unmodeled dynamics are present. Consider

$$
\begin{aligned}
& \dot{\mathbf{x}}=\mathbf{A} \mathbf{x}+\left(\begin{array}{l}
1 \\
1
\end{array}\right) \theta+\left(\begin{array}{l}
0 \\
1
\end{array}\right) \lambda, \mathbf{A}=\left(\begin{array}{ll}
-a_{1} & 1 \\
-a_{2} & 0
\end{array}\right) \\
& y=x_{1}, a_{1}, a_{2} \in \mathbb{R}_{>0} .
\end{aligned}
$$

Let $\mathbf{x}\left(t, \theta, \lambda, \mathbf{x}_{0}\right)$ and $\mathbf{x}\left(t, \theta^{\prime}, \lambda^{\prime}, \mathbf{x}_{0}^{\prime}\right)$ be two solutions of (15) corresponding to different parameter values and initial conditions, and let $\mathbf{e}=\operatorname{col}\left(e_{1}, e_{2}\right)=\mathbf{x}\left(t, \theta, \lambda, \mathbf{x}_{0}\right)-$ $\mathbf{x}\left(t, \theta^{\prime}, \lambda^{\prime}, \mathbf{x}_{0}^{\prime}\right)$. Picking $e_{2}\left(t_{0}\right)=-\theta+\theta^{\prime}, e_{1}\left(t_{0}\right)=0$ ensures that $e_{1}(t)=0$ for all $t \geq t_{0}$ if $\theta-\theta^{\prime}+\lambda-\lambda^{\prime}=0$. Another, albeit nonlinearly parameterized, example is

$$
\dot{x}=-x+\theta+\left[\sin ^{2}(\lambda+t)+x^{2}+1\right]^{-1}, y=x .
$$

In this case $x\left(t, \theta, \lambda, x_{0}\right)=x\left(t, \theta, \lambda^{\prime}, x_{0}\right)$ for all $t \geq t_{0}$ if $\lambda^{\prime}=\lambda+k \pi, k \in \mathbb{Z}, \theta^{\prime}=\theta$.

In order to account for possible non-unique parametrization, for each pair $\boldsymbol{\theta}, \boldsymbol{\lambda}$ we introduce two sets: $\mathcal{E}_{0}(\boldsymbol{\lambda}, \boldsymbol{\theta})$ and $\mathcal{E}(\boldsymbol{\lambda}, \boldsymbol{\theta})$. The set $\mathcal{E}_{0}(\boldsymbol{\lambda}, \boldsymbol{\theta})$ :

$$
\begin{gathered}
\mathcal{E}_{0}(\boldsymbol{\lambda}, \boldsymbol{\theta})=\left\{\left(\boldsymbol{\lambda}^{\prime}, \boldsymbol{\theta}^{\prime}\right), \boldsymbol{\lambda}^{\prime} \in \mathbb{R}^{p}, \boldsymbol{\theta}^{\prime} \in \mathbb{R}^{m} \mid\right. \\
\left.\boldsymbol{\eta}_{0}\left(t, \boldsymbol{\lambda}, \boldsymbol{\theta}, \boldsymbol{\lambda}^{\prime}, \boldsymbol{\theta}^{\prime}\right)=0, \forall t \geq t_{0}\right\}, \\
\boldsymbol{\eta}_{0}\left(t, \boldsymbol{\lambda}, \boldsymbol{\theta}, \boldsymbol{\lambda}^{\prime}, \boldsymbol{\theta}^{\prime}\right)= \\
\mathbf{B}\left(\boldsymbol{\varphi}^{T}(t, \boldsymbol{\lambda}, y(t)) \boldsymbol{\theta}-\boldsymbol{\varphi}^{T}\left(t, \boldsymbol{\lambda}^{\prime}, y(t)\right) \boldsymbol{\theta}^{\prime}\right)+ \\
\mathbf{g}\left(t, \boldsymbol{\lambda}^{\prime}, y(t), u(t)\right)-\mathbf{g}(t, \boldsymbol{\lambda}, y(t), u(t))
\end{gathered}
$$

contains all parametrizations of (7) that are indistinguishable from observations of $\mathbf{x}(t)$ for the given $y(\cdot), u(\cdot), \boldsymbol{\theta}, \boldsymbol{\lambda}$ at $\boldsymbol{\xi}(t) \equiv 0$. That is, if $\mathbf{x}\left(t, \boldsymbol{\theta}, \boldsymbol{\lambda}, \mathbf{x}_{0}\right)-$ $\mathbf{x}\left(t, \boldsymbol{\theta}^{\prime}, \boldsymbol{\lambda}^{\prime}, \mathbf{x}_{0}\right)=0$ for all $t \geq t_{0}$ then $\left(\boldsymbol{\lambda}^{\prime}, \boldsymbol{\theta}^{\prime}\right) \in \mathcal{E}_{0}(\boldsymbol{\lambda}, \boldsymbol{\theta})$. For system (15) the set $\mathcal{E}_{0}(\lambda, \theta)$ contains just one element, $(\lambda, \theta)$. The set $\mathcal{E}_{0}(\lambda, \theta)$, however, is not finite for system (16) and for parametrization (13) of the first model in Table 1.

The second set, $\mathcal{E}(\boldsymbol{\lambda}, \boldsymbol{\theta})$, is defined as:

$$
\begin{aligned}
& \mathcal{E}(\boldsymbol{\lambda}, \boldsymbol{\theta})=\left\{\left(\boldsymbol{\lambda}^{\prime}, \boldsymbol{\theta}^{\prime}\right), \boldsymbol{\lambda}^{\prime} \in \mathbb{R}^{p}, \boldsymbol{\theta}^{\prime} \in \mathbb{R}^{m} \mid \exists \mathbf{p}\left(\boldsymbol{\theta}, \boldsymbol{\lambda}, \boldsymbol{\theta}^{\prime}, \boldsymbol{\lambda}^{\prime}\right)\right. \\
& \left.\in \mathbb{R}^{n-1}: \tilde{\mathbf{C}}^{T} e^{\Lambda\left(t-t_{0}\right)} \mathbf{p}+\boldsymbol{\eta}\left(t, \boldsymbol{\theta}, \boldsymbol{\lambda}, \boldsymbol{\theta}^{\prime}, \boldsymbol{\lambda}^{\prime}\right)=0 \quad \forall t \geq t_{0}\right\}
\end{aligned}
$$

where $\tilde{\mathbf{C}} \in \mathbb{R}^{n-1}, \tilde{\mathbf{C}}=\operatorname{col}(1,0, \ldots, 0)$,

$$
\begin{aligned}
& \boldsymbol{\eta}\left(t, \boldsymbol{\lambda}, \boldsymbol{\theta}, \boldsymbol{\lambda}^{\prime}, \boldsymbol{\theta}^{\prime}\right)=\boldsymbol{\varphi}(t, \boldsymbol{\lambda}, y(t))^{T} \boldsymbol{\theta}-\boldsymbol{\varphi}\left(t, \boldsymbol{\lambda}^{\prime}, y(t)\right)^{T} \boldsymbol{\theta}^{\prime}+ \\
& g_{1}(t, \boldsymbol{\lambda}, y(t), u(t))-g_{1}\left(t, \boldsymbol{\lambda}^{\prime}, y(t), u(t)\right)+q\left(t, \boldsymbol{\lambda}, \boldsymbol{\lambda}^{\prime}\right)(19)
\end{aligned}
$$

and $q\left(t, \boldsymbol{\lambda}, \boldsymbol{\lambda}^{\prime}\right)=\tilde{\mathbf{C}}^{T} \mathbf{z}\left(t, \boldsymbol{\lambda}, \boldsymbol{\lambda}^{\prime}\right)$ :

$$
\begin{aligned}
& \dot{\mathbf{z}}=\Lambda \mathbf{z}+\mathbf{G}\left(\mathbf{g}(t, \boldsymbol{\lambda}, y(t), u(t))-\mathbf{g}\left(t, \boldsymbol{\lambda}^{\prime}, y(t), u(t)\right)\right), \\
& \Lambda=\left(\begin{array}{c|c}
-\mathbf{b} & I_{n-2} \\
0
\end{array}\right), \mathbf{G}=\left(\begin{array}{l}
-\mathbf{b} I_{n-1}
\end{array}\right) \\
& \mathbf{z}\left(t_{0}\right)=0, \mathbf{b}=\operatorname{col}\left(b_{1}, \ldots, b_{n-1}\right) .
\end{aligned}
$$

If $\boldsymbol{\xi}(t) \equiv 0$ then the set $\mathcal{E}(\boldsymbol{\lambda}, \boldsymbol{\theta})$ contains all indistinguishable parametrizations of $(7)$ for the given $y(\cdot), u(\cdot), \boldsymbol{\theta}$, $\boldsymbol{\lambda}$ (see Lemma 12 in Section 5), and $\mathcal{E}_{0}(\boldsymbol{\lambda}, \boldsymbol{\theta}) \subseteq \mathcal{E}(\boldsymbol{\lambda}, \boldsymbol{\theta})$. Note that if $\operatorname{dim}(\mathbf{x})=2$ then $\Lambda=-b_{1}, \mathbf{G}=\left(-b_{1} 1\right)$; if $\operatorname{dim}(\mathbf{x})=1$ we set $\mathcal{E}(\boldsymbol{\lambda}, \boldsymbol{\theta})=\mathcal{E}_{0}(\boldsymbol{\lambda}, \boldsymbol{\theta})$. For system (15), $\mathcal{E}(\lambda, \theta)=\left\{\left(\lambda^{\prime}, \theta^{\prime}\right), \lambda \in \mathbb{R}, \theta \in \mathbb{R} \mid \theta-\theta^{\prime}+\lambda-\lambda^{\prime}=0\right\}$. Note that if $\mathbf{g}(t, \boldsymbol{\lambda}, y(t), u(t))=\mathbf{B} g(t, \boldsymbol{\lambda}, y(t), u(t)), \mathbf{B}=$ $\operatorname{col}\left(1, b_{1}, \ldots, b_{n-1}\right)$, then $q\left(t, \boldsymbol{\lambda}, \boldsymbol{\lambda}^{\prime}\right) \equiv 0$ for all $t \geq t_{0}$ in (19). The introduction of sets $\mathcal{E}_{0}(\boldsymbol{\lambda}, \boldsymbol{\theta}), \mathcal{E}(\boldsymbol{\lambda}, \boldsymbol{\theta})$ does not, of course, resolve identifiability issues. It helps, however, to specify constraints on the nonlinearities in $(7)$ for which the parameter reconstruction, up to $\mathcal{E}_{0}, \mathcal{E}$, can be achieved.

Since this is desirable from an implementation point of view, we will seek for a recursive procedure taking the values $y(t), u(t)$ as inputs and producing the estimates $\hat{\mathbf{x}}(t), \hat{\boldsymbol{\theta}}(t), \hat{\boldsymbol{\lambda}}(t)$ of $\mathbf{x}\left(t, \boldsymbol{\lambda}, \boldsymbol{\theta}, \mathbf{x}_{0},[u]\right), \boldsymbol{\theta}$, and $\boldsymbol{\lambda}$, respectively, as outputs. Note that since we allow for nonidentifiable configurations, estimation of parameters $\boldsymbol{\theta}$, $\boldsymbol{\lambda}$ is possible only up to the set $\mathcal{E}(\boldsymbol{\lambda}, \boldsymbol{\theta})$. We will, therefore, be looking for an auxiliary system, i.e. an adaptive observer: $\dot{\mathbf{q}}=\mathbf{f}(t, y, u(t), \mathbf{q}), \mathbf{f}: \mathbb{R} \times \mathbb{R} \times \mathbb{R} \times \mathbb{R}^{q} \rightarrow \mathbb{R}^{q}$, $\mathbf{q}\left(t_{0}\right)=\mathbf{q}_{0}, \mathbf{q} \in \mathbb{R}^{q}$, and functions $\mathbf{h}_{\theta}: \mathbb{R}^{q} \rightarrow \mathbb{R}^{m}$, $\mathbf{h}_{\lambda}: \mathbb{R}^{q} \rightarrow \mathbb{R}^{p}, \mathbf{h}_{x}: \mathbb{R}^{q} \rightarrow \mathbb{R}^{n}$ such that for the given $t_{0}$, appropriately chosen $\mathbf{q}_{0}$, known functions $r_{1}, r_{2} \in \mathcal{K}$, and all admissible $\boldsymbol{\lambda}, \boldsymbol{\theta}, \mathbf{x}_{0}$ the following requirements hold for the observer:

$$
\begin{aligned}
& \limsup _{t \rightarrow \infty}\left\|\mathbf{h}_{x}\left(\mathbf{q}\left(t, \mathbf{q}_{0}\right)\right)-\mathbf{x}\left(t, \boldsymbol{\lambda}, \boldsymbol{\theta}, \mathbf{x}_{0}\right)\right\| \leq r_{1}\left(\Delta_{\xi}\right) \\
& \underset{t \rightarrow \infty}{\limsup } \operatorname{dist}\left(\left(\begin{array}{c}
\mathbf{h}_{\lambda}\left(\mathbf{q}\left(t, \mathbf{q}_{0}\right)\right) \\
\mathbf{h}_{\theta}\left(\mathbf{q}\left(t, \mathbf{q}_{0}\right)\right)
\end{array}\right), \mathcal{E}(\boldsymbol{\lambda}, \boldsymbol{\theta})\right) \leq r_{2}\left(\Delta_{\xi}\right),
\end{aligned}
$$

where $\Delta_{\xi}$ is defined in (8).

\section{Main result}

In this section we introduce an observer for (7) and show that asymptotic reconstruction of state and parameters of (7) is achievable (up to the set $\mathcal{E}(\boldsymbol{\lambda}, \boldsymbol{\theta})$ ), subject to some persistency of excitation conditions.

\subsection{Observer definition}

Following the general ideas of [40] with regards to the treatment of uncertain systems with general nonlinear 


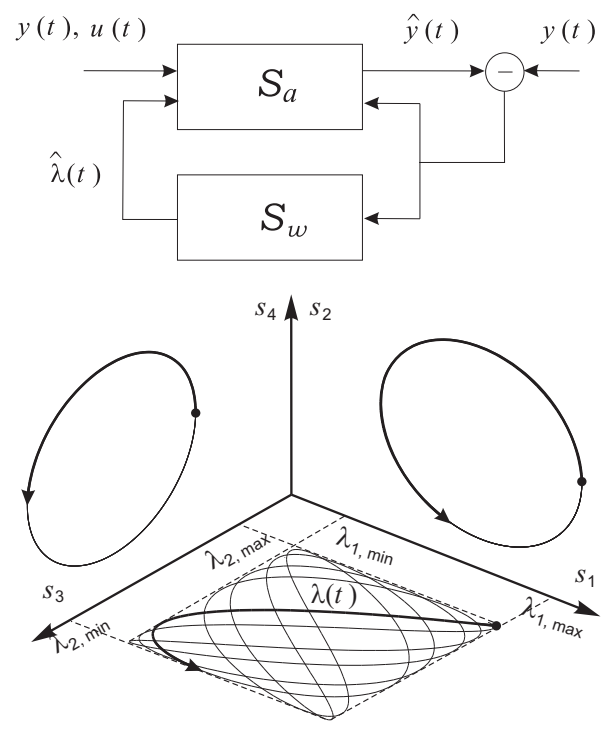

Figure 1. Top panel: general structure of the observer. Bottom panel: phase curves of system (25).

parametrization, we propose that an asymptotically converging observer for (7) consists of two coupled subsystems, $\mathcal{S}_{a}$ and $\mathcal{S}_{w}$ (see Fig. 1). The role of subsystem $\mathcal{S}_{a}$ is to provide estimates of state and parameters $\boldsymbol{\theta}$ of (7), and the role of subsystem $\mathcal{S}_{w}$ is to search the values of parameters $\boldsymbol{\lambda}$.

The dynamics of subsystem $\mathcal{S}_{a}$ is defined as follows:

$$
\mathcal{S}_{a}:\left\{\begin{aligned}
\dot{\hat{\mathbf{x}}}=\mathbf{A} \hat{\mathbf{x}}+\boldsymbol{\ell}\left(\mathbf{C}^{T} \hat{\mathbf{x}}-y(t)\right)+\mathbf{B} \boldsymbol{\varphi}^{T}(t, \hat{\boldsymbol{\lambda}}(t), y(t)) \hat{\boldsymbol{\theta}} & \\
& +\mathbf{g}(t, \hat{\boldsymbol{\lambda}}(t), y(t), u(t)) \\
\dot{\hat{\boldsymbol{\theta}}=} & -\gamma_{\theta}\left(\mathbf{C}^{T} \hat{\mathbf{x}}-y(t)\right) \boldsymbol{\varphi}(t, \hat{\boldsymbol{\lambda}}(t), y(t)), \\
\hat{y}= & \mathbf{C}^{T} \hat{\mathbf{x}}, \hat{\mathbf{x}}\left(t_{0}\right) \in \mathbb{R}^{n}, \hat{\boldsymbol{\theta}}\left(t_{0}\right) \in \mathbb{R}^{m}, \gamma_{\theta} \in \mathbb{R}_{>0}
\end{aligned}\right.
$$

The variable $\hat{\boldsymbol{\theta}}=\operatorname{col}\left(\hat{\theta}_{1}, \cdots, \hat{\theta}_{m}\right)$ in $(22)$ is an estimate of $\boldsymbol{\theta}$, and $\hat{\boldsymbol{\lambda}}=\operatorname{col}\left(\hat{\lambda}_{1}, \ldots, \hat{\lambda}_{p}\right)$ is an estimate of $\boldsymbol{\lambda}$. For the time being we suppose that $\hat{\lambda}$ is a continuous function of $t$. The matrix $\mathbf{A}$ and vectors $\mathbf{B}, \mathbf{C}$ in (22) are identical to those in (7), and the vector $\boldsymbol{\ell}$ in (22) satisfies Assumption 3.1 . If the values of $\boldsymbol{\lambda}$ would be known then substitution $\hat{\boldsymbol{\lambda}}=\boldsymbol{\lambda}$ reduces system (22) to (3), and conditions for asymptotic reconstruction of state and parameter values of (7) follow from Theorem 3. The values of $\boldsymbol{\lambda}$, however, are unknown and therefore a procedure for estimating the values of $\boldsymbol{\lambda}$ is needed.

Regarding the definition of $\mathcal{S}_{w}$, we propose that the values of $\hat{\boldsymbol{\lambda}}(t)$ result from an explorative search in the domain $\Omega_{\lambda}$ of the admissible values for $\boldsymbol{\lambda}$. The exploration can be realized by movements along the solutions of a certain class of dynamical systems. Let us, for instance, consider systems governed by the following equations

$$
\dot{\mathbf{s}}=\mathbf{f}(\mathbf{s}), \mathbf{s}\left(t_{0}\right)=\mathbf{s}_{0}, \hat{\lambda}=\boldsymbol{\beta}(\mathbf{s})
$$

where $\mathbf{f}: \mathbb{R}^{n_{p}} \rightarrow \mathbb{R}^{n_{p}}, \boldsymbol{\beta}: \mathbb{R}^{n_{p}} \rightarrow \mathbb{R}^{p}$ are continuous, and let $\Omega_{s}$ be the $\omega$-limit set ${ }^{2}$ of $\mathbf{s}_{0}$. In addition, suppose that the following properties hold:

P1) the functions $\mathbf{f}, \boldsymbol{\beta}$ in (23) are Lipschitz;

P2) the vector $\mathbf{s}_{0}$ is such that the solution $\mathbf{s}\left(t, \mathbf{s}_{0}\right)$ is bounded for all $t \geq t_{0}$;

P3) the image of $\Omega_{s}$ under $\boldsymbol{\beta}$ contains $\Omega_{\lambda}$ : for every $\boldsymbol{\lambda} \in \Omega_{\lambda}$ there is an $\mathbf{s} \in \Omega_{s}$ such that $\boldsymbol{\beta}(\mathbf{s})=\boldsymbol{\lambda}$.

Properties $\mathrm{P} 1$ and $\mathrm{P} 2$ are technical requirements ensuring that the derivative of $\hat{\lambda}$, as a function of $t$, is bounded and has a bounded growth rate. Property P3, however, is essential. It implies that the projection $\boldsymbol{\beta}\left(\mathbf{s}\left(\cdot, \mathbf{s}_{0}\right)\right)$ of the trajectory $\mathbf{s}\left(\cdot, \mathbf{s}_{0}\right)$ onto $\Omega_{\lambda}$ is dense and recurring in $\Omega_{\lambda}$ :

$$
\begin{aligned}
\forall \boldsymbol{\lambda} \in \Omega_{\lambda}, & \forall \varepsilon \in \mathbb{R}_{>0}, \forall t \geq t_{0} \\
& \exists t^{\prime}>t:\left\|\boldsymbol{\lambda}-\boldsymbol{\beta}\left(\mathbf{s}\left(t^{\prime}, \mathbf{s}_{0}\right)\right)\right\|<\varepsilon .
\end{aligned}
$$

Indeed, let $\lambda^{\prime}$ be an element from $\Omega_{\lambda}$. Then according to P3 there is an $\mathbf{s}^{\prime} \in \Omega_{s}: \boldsymbol{\beta}\left(\mathbf{s}^{\prime}\right)=\boldsymbol{\lambda}^{\prime}$. Since $\Omega_{s}$ is the $\omega$-limit set of $\mathbf{s}_{0}$, we can conclude that there is a sequence $\left\{t_{i}\right\}, i=1,2, \ldots, \lim _{i \rightarrow \infty} t_{i}=\infty$, such that $\lim _{i \rightarrow \infty} \mathbf{s}\left(t_{i}, \mathbf{s}_{0}\right)=\mathbf{s}^{\prime}$. Finally, using the continuity of $\boldsymbol{\beta}$ we arrive at $\lim _{i \rightarrow \infty} \boldsymbol{\beta}\left(\mathbf{s}\left(t_{i}, \mathbf{s}_{0}\right)\right)=\boldsymbol{\lambda}^{\prime}$. In other words, for any $\boldsymbol{\lambda}^{\prime} \in \Omega_{\lambda}$ and $\varepsilon>0$ there will exist a sequence of time instances $t_{i}: \lim _{i \rightarrow \infty} t_{i}=\infty$ such that $\left\|\hat{\boldsymbol{\lambda}}\left(t_{i}\right)-\boldsymbol{\lambda}^{\prime}\right\|<\varepsilon$, and hence (24) follows. An example of a very simple system possessing a solution $\mathbf{s}\left(t, \mathbf{s}_{0}\right)$ and an output function $\boldsymbol{\beta}$ satisfying properties $\mathrm{P} 1-\mathrm{P} 3$ for $\Omega_{\lambda}=[-1,1]^{2}$ is

$$
\begin{aligned}
& \dot{s}_{1}=-\sqrt{2} s_{2}, \dot{s}_{2}=\sqrt{2} s_{1}, \dot{s}_{3}=-s_{4}, \dot{s}_{4}=s_{3}, \\
& \boldsymbol{\beta}\left(\mathbf{s}\left(t, \mathbf{s}_{0}\right)\right)=\operatorname{col}\left(s_{1}(t), s_{3}(t)\right), \mathbf{s}_{0}=\operatorname{col}(1,0,1,0) .
\end{aligned}
$$

Phase curves of (25) are shown in Fig. 1, bottom panel. Projections of the initial segment of the trajectory are shown by thick lines. After evolving beyond the initial segment, the values of $\boldsymbol{\beta}\left(\mathbf{s}\left(t, \mathbf{s}_{0}\right)\right)$ will densely fill the set $\left[\lambda_{1, \min }, \lambda_{1, \max }\right] \times\left[\lambda_{2, \min }, \lambda_{2, \max }\right]=[-1,1]^{2}$, cf. [33].

The problem with using (23) directly as an estimator for $\lambda$ is that exploration of the set $\Omega_{\lambda}$ continues indefinitely. For the purposes of observer design we need to ensure that exploration of $\Omega_{\lambda}$ stops once a sufficiently small neighborhood of the set $\mathcal{E}(\boldsymbol{\lambda}, \boldsymbol{\theta})$ has been reached. To enable this, the explorative subsystem must be supplied with an error measure. A function of $\|y(t)-\hat{y}(t)\|_{\varepsilon}$ is a possible candidate for such a measure. Thus we replace the earlier definition (23) for $\hat{\boldsymbol{\lambda}}$ with the following:

$$
\begin{aligned}
& \dot{\mathbf{s}}=\gamma \sigma\left(\|y(t)-\hat{y}(t)\|_{\varepsilon}\right) \mathbf{f}(\mathbf{s}), \varepsilon \in \mathbb{R}_{\geq 0}, \gamma \in \mathbb{R}_{>0}, \\
& \hat{\boldsymbol{\lambda}}=\boldsymbol{\beta}(\mathbf{s}), \mathbf{s}\left(t_{0}\right)=\mathbf{s}_{0},
\end{aligned}
$$

2 Recall that a point $\mathbf{z} \in \mathbb{R}^{n_{p}}$ is an $\omega$-limit point of $\mathbf{z}_{0} \in \mathbb{R}^{n_{p}}$ if there is a sequence $\left\{t_{i}\right\}, i=1,2, \ldots, \lim _{i \rightarrow \infty} t_{i}=\infty$, such that $\lim _{i \rightarrow \infty} \mathbf{s}\left(t_{i}, \mathbf{z}_{0}\right)=\mathbf{z}$. The set of all $\omega$-limit points of $\mathbf{z}_{0}$ is the $\omega$-limit set of $\mathbf{z}_{0}$. 
where $\sigma: \mathbb{R}_{\geq 0} \rightarrow \mathbb{R}_{\geq 0}$ is a bounded Lipschitz function:

$$
\begin{aligned}
\exists D_{\sigma}, & M_{\sigma} \in \mathbb{R}_{>0}: \\
& \sigma(v) \leq M_{\sigma}, \sigma(v) \leq D_{\sigma} v \forall v \geq 0
\end{aligned}
$$

such that $\sigma(v)>0$ for $v>0$, and $\sigma(0)=0$.

For the sake of simplicity and without loss of generality, instead of dealing with general systems (26), we will focus on a specific system of equations:

$$
\begin{gathered}
\mathcal{S}_{w}:\left\{\begin{aligned}
\dot{s}_{2 j-1}= & \gamma \sigma\left(\|y(t)-\hat{y}(t)\|_{\varepsilon}\right) \cdot \omega_{j} \cdot\left(s_{2 j-1}-s_{2 j}\right. \\
& \left.-s_{2 j-1}\left(s_{2 j-1}^{2}+s_{2 j}^{2}\right)\right) \\
\dot{s}_{2 j}=\quad & \gamma \sigma\left(\|y(t)-\hat{y}(t)\|_{\varepsilon}\right) \cdot \omega_{j} \cdot\left(s_{2 j-1}+s_{2 j}\right. \\
& \left.-s_{2 j}\left(s_{2 j-1}^{2}+s_{2 j}^{2}\right)\right) \\
& =\beta_{j}(\mathbf{s}), j=\{1, \ldots, p\}
\end{aligned}\right. \\
\hat{\lambda}_{j}(\mathbf{s})=\lambda_{j, \min }+\frac{\lambda_{j, \max }-\lambda_{j, \min }}{2}\left(s_{2 j-1}+1\right)
\end{gathered}
$$

where $\sigma$ is a function satisfying (27). Parameters $\omega_{j} \in$ $\mathbb{R}_{>0}$ in (28) are supposed to be rationally-independent:

$$
\sum_{j=1}^{p} \omega_{j} k_{j} \neq 0, \forall k_{j} \in \mathbb{Z}
$$

Equations (28)-(30) are straightforward generalizations from the example system of which the phase curves are shown in Fig. 1. If the term $\gamma \sigma\left(\|y(t)-\hat{y}(t)\|_{\varepsilon}\right)$ in the right-hand side of $(28)$ is substituted with 1 , these equations satisfy the requirements P1-P3. Indeed, we can immediately see that in this case $\left(s_{2 j-1}\left(t, \mathbf{s}_{0}\right), s_{2 j}\left(t, \mathbf{s}_{0}\right)\right)=$ $\left(\cos \left(\omega_{j}\left(t-t_{0}\right)+a_{j}\right), \sin \left(\omega_{j}\left(t-t_{0}\right)+a_{j}\right)\right), a_{j} \in \mathbb{R}$. Thus properties P1, P2 hold. Trajectories $s_{1}\left(\cdot, \mathbf{s}_{0}\right), s_{3}\left(\cdot, \mathbf{s}_{0}\right)$, $\ldots s_{2 p-1}\left(\cdot, \mathbf{s}_{0}\right)$ evolve on a corresponding $p$-dimensional invariant torus. Since $\omega_{j}$ are rationally-independent these trajectories densely fill the torus (cf. [3], [33], [20]) or, alternatively, they densely fill the hypercube $[-1,1]^{p}$. This implies that $\Omega_{s}$, the $\omega$-limit set of $\mathbf{s}_{0}$, is $\Omega_{s}=\left\{\operatorname{col}\left(s_{1}, s_{2}, \ldots, s_{2 p}\right) \in \mathbb{R}^{p} \mid \operatorname{col}\left(s_{1}, s_{3}, \ldots, s_{2 p-1}\right) \in\right.$ $\left.[-1,1]^{p}, s_{2 j}= \pm \sqrt{1-s_{2 j-1}^{2}}, j=1, \ldots, p\right\}$. Noticing that the image of $\Omega_{s}$ under transformation $\boldsymbol{\beta}$ coincides with $\Omega_{\lambda}$ we conclude that P3 holds.

Concerning the structure of $\mathcal{S}_{w}$, no additional modeldependent constraints are imposed on (28) (or, in general, on (26)), apart from the general requirements P1P3. Model-specific nonlinearities are accounted for in the "converging" part, $\mathcal{S}_{a}$, of the observer producing the estimates for $\boldsymbol{\theta}$ and $\mathbf{x}$. The information about the values of $\boldsymbol{\lambda}$ is transferred to the exploratory part, $\mathcal{S}_{w}$, by means of $\|y(t)-\hat{y}(t)\|_{\varepsilon}$. The latter variable modulates the speed of exploration in $\Omega_{\lambda}$ along a search trajectory.
The search trajectory itself does not need to be dependent on the properties of $\mathbf{g}, \boldsymbol{\varphi}$, and neither is the structure of $\mathcal{S}_{w}$. This potential advantage of the approach, however, comes at a cost. According to (24), small neighborhoods of sets to which the solutions of the combined system (22), (28) converge are not necessarily forward invariant. Hence these sets are not guaranteed to be asymptotically stable. Nevertheless, albeit in a weaker sense [31], they are still attracting. We illustrate this point in Section 7.

\subsection{Asymptotic properties of the observer}

Let us now proceed with specifying those properties of (7) that can be useful for state and parameter reconstruction. Recall that (7) is a generalization of the standard canonic observer form (1). According to Theorem 3 , one of the conditions for (3) to be an adaptive observer for (1) is persistency of excitation of the restriction of $\phi(\cdot, y(\cdot))$ on $\mathbb{R}_{\geq t_{0}}$. It is therefore natural to expect that some kind of persistency of excitation conditions might be needed for reconstruction of parameters $\boldsymbol{\theta}, \boldsymbol{\lambda}$ in (7) too. Two versions of these conditions will be considered, namely the notions of uniform persistency of excitation [24] and nonlinear persistency of excitation [8].

Definition 4 A function $\boldsymbol{\alpha}: \mathbb{R}_{\geq t_{0}} \times \Omega_{\lambda} \rightarrow \mathbb{R}^{p}$ is $\lambda$ Uniformly Persistently Exciting $(\bar{\lambda}$-UPE with $T, \mu)$, denoted by $\boldsymbol{\alpha}(t, \boldsymbol{\lambda}) \in \lambda \mathrm{UPE}(T, \mu)$, if there exist $T, \mu \in \mathbb{R}_{>0}$ :

$$
\int_{t}^{t+T} \boldsymbol{\alpha}(\tau, \boldsymbol{\lambda}) \boldsymbol{\alpha}^{T}(\tau, \boldsymbol{\lambda}) \mathrm{d} \tau \geq \mu \mathbf{I}_{p}, \forall t \geq t_{0}, \boldsymbol{\lambda} \in \Omega_{\lambda} .
$$

In contrast to the conventional definitions of persistency of excitation (cf. Definition 2), uniform persistency of excitation requires existence of $\mu, T \in \mathbb{R}_{>0}$ in (31) that are independent on $\boldsymbol{\lambda}$ for all $\boldsymbol{\lambda} \in \Omega_{\lambda}$. This is a stronger restriction; we will, however, require that it holds for $\boldsymbol{\varphi}(t, \boldsymbol{\lambda}, y(t))$ (as a function of $t, \boldsymbol{\lambda}$ on $\mathbb{R}_{\geq t_{0}} \times \Omega_{\lambda}$ ) in (7).

Since parametrization of (7) is allowed to be nonlinear, it is natural to expect that reconstruction of model parameters might require a nonlinear version of standard persistency of excitation. Here we employ the following generalization of the standard notion (cf. [8]):

Definition 5 Let $\mathcal{E}$ be a set-valued map defined on $\mathcal{D} \subset$ $\mathbb{R}^{d}$ and associating a subset of $\mathcal{D}$ to every $\mathbf{p} \in \mathcal{D}$. A function $\boldsymbol{\alpha}: \mathbb{R}_{\geq t_{0}} \times \mathcal{D} \times \mathcal{D} \rightarrow \mathbb{R}^{k}$ is said to be weakly Nonlinearly Persistently Exciting in $\mathbf{p}$ wrt $\mathcal{E}$ (wNPE with $L, \beta, \mathcal{E})$, denoted by $\boldsymbol{\alpha}\left(t, \mathbf{p}, \mathbf{p}^{\prime}\right) \in \mathrm{wNPE}(L, \beta, \mathcal{E})$, if there exist $L \in \mathbb{R}_{>0}, t_{1} \geq t_{0}$, and $\beta \in \mathcal{K}_{\infty}$ :

$$
\begin{gathered}
\forall t \geq t_{1}, \mathbf{p}, \mathbf{p}^{\prime} \in \mathcal{D} \quad \exists t^{\prime} \in[t, t+L]: \\
\left\|\boldsymbol{\alpha}\left(t^{\prime}, \mathbf{p}, \mathbf{p}^{\prime}\right)\right\| \geq \beta\left(\operatorname{dist}\left(\mathcal{E}(\mathbf{p}), \mathbf{p}^{\prime}\right)\right) .
\end{gathered}
$$


If the set $\mathcal{E}(\mathbf{p})$ contains just one element, $\mathbf{p}$, then the inequality in (32) reduces to $\left\|\boldsymbol{\alpha}\left(t^{\prime}, \mathbf{p}, \mathbf{p}^{\prime}\right)\right\| \geq \beta\left(\left\|\mathbf{p}-\mathbf{p}^{\prime}\right\|\right)$. Taking the above notions into account we formulate the main technical assumption on the nonlinearities in (7):

Assumption 4.1 The functions $\boldsymbol{\varphi}, \mathbf{g}$ in the right-hand side of (7) are such that

A1) the restriction of the function $\boldsymbol{\alpha}_{1}: \mathbb{R} \times \mathbb{R}^{p} \rightarrow \mathbb{R}^{m}$, $\boldsymbol{\alpha}_{1}(t, \boldsymbol{\lambda})=\boldsymbol{\varphi}(t, \boldsymbol{\lambda}, y(t))$ on $\mathbb{R}_{\geq t_{0}} \times \Omega_{\lambda}$ is $\lambda$-UPE with $T, \mu$;

A2) the restriction of $\boldsymbol{\alpha}_{2}: \mathbb{R} \times \mathbb{R}^{p+m} \times \mathbb{R}^{p+m} \rightarrow \mathbb{R}$, $\boldsymbol{\alpha}_{2}\left(t,(\boldsymbol{\lambda}, \boldsymbol{\theta}),\left(\boldsymbol{\lambda}^{\prime}, \boldsymbol{\theta}^{\prime}\right)\right)=\boldsymbol{\eta}\left(t, \boldsymbol{\lambda}, \boldsymbol{\theta}, \boldsymbol{\lambda}^{\prime}, \boldsymbol{\theta}^{\prime}\right)$, where $\boldsymbol{\eta}(\cdot)$ is defined in (19), on $\mathbb{R}_{\geq t_{0}} \times \mathbb{R}^{p+m} \times \mathbb{R}^{p+m}$ is weakly nonlinearly persistently exciting in $(\boldsymbol{\lambda}, \boldsymbol{\theta})$ wrt to the map $\mathcal{E}(\boldsymbol{\lambda}, \boldsymbol{\theta})$ determined by (18).

Remark 6 Checking that condition A1 holds is straightforward if e.g. $\boldsymbol{\varphi}(t, \boldsymbol{\lambda}, y(t))$ is periodic in $t$. Regarding condition A2, we note that, according to (19), $\boldsymbol{\eta}\left(t, \boldsymbol{\lambda}, \boldsymbol{\theta}, \boldsymbol{\lambda}^{\prime}, \boldsymbol{\theta}^{\prime}\right)$ can be expressed as $\boldsymbol{\eta}\left(t, \boldsymbol{\lambda}, \boldsymbol{\theta}, \boldsymbol{\lambda}^{\prime}, \boldsymbol{\theta}^{\prime}\right)=$ $r(t, \boldsymbol{\lambda}, \boldsymbol{\theta})-r\left(t, \boldsymbol{\lambda}^{\prime}, \boldsymbol{\theta}\right)+\boldsymbol{\varphi}\left(t, \boldsymbol{\lambda}^{\prime}, y(t)\right)^{T}\left(\boldsymbol{\theta}-\boldsymbol{\theta}^{\prime}\right)$, where

$$
\begin{aligned}
& r(t, \boldsymbol{\lambda}, \boldsymbol{\theta})=\boldsymbol{\varphi}(t, \boldsymbol{\lambda}, y(t))^{T} \boldsymbol{\theta}+g_{1}(t, \boldsymbol{\lambda}, y(t), u(t)) \\
& +\tilde{\mathbf{C}}^{T} \int_{t_{0}}^{t} e^{\Lambda(t-\tau)} \mathbf{G g}(\tau, \boldsymbol{\lambda}, y(\tau), u(\tau)) d \tau .
\end{aligned}
$$

If $\boldsymbol{\varphi}, \mathbf{g}$ are differentiable then $r(t, \boldsymbol{\lambda}, \boldsymbol{\theta})-r\left(t, \boldsymbol{\lambda}^{\prime}, \boldsymbol{\theta}\right)=$ $\mathbf{R}\left(t, \boldsymbol{\lambda}, \boldsymbol{\lambda}^{\prime}, \boldsymbol{\theta}\right)\left(\boldsymbol{\lambda}-\boldsymbol{\lambda}^{\prime}\right)$, where $\mathbf{R}\left(t, \boldsymbol{\lambda}, \boldsymbol{\lambda}^{\prime}, \boldsymbol{\theta}\right)=\int_{0}^{1} \frac{\partial}{\partial \mathbf{s}} r(t$ $\left., \mathbf{s}\left(\xi, \boldsymbol{\lambda}, \boldsymbol{\lambda}^{\prime}\right), \boldsymbol{\theta}\right) d \xi, \mathbf{s}\left(\xi, \boldsymbol{\lambda}, \boldsymbol{\lambda}^{\prime}\right)=\boldsymbol{\lambda} \xi+(1-\xi) \boldsymbol{\lambda}^{\prime}$. Hence

$$
\begin{aligned}
& \boldsymbol{\eta}\left(t, \boldsymbol{\lambda}, \boldsymbol{\theta}, \boldsymbol{\lambda}^{\prime}, \boldsymbol{\theta}^{\prime}\right)= \\
& \quad\left(\boldsymbol{\varphi}^{T}\left(t, \boldsymbol{\lambda}^{\prime}, y(t)\right), \mathbf{R}\left(t, \boldsymbol{\lambda}, \boldsymbol{\lambda}^{\prime}, \boldsymbol{\theta}\right)\right)\left(\boldsymbol{\theta}-\boldsymbol{\theta}^{\prime}, \boldsymbol{\lambda}-\boldsymbol{\lambda}^{\prime}\right)
\end{aligned}
$$

It is therefore clear that if $\left(\boldsymbol{\varphi}^{T}\left(t, \boldsymbol{\lambda}^{\prime}, y(t)\right), \mathbf{R}\left(t, \boldsymbol{\lambda}, \boldsymbol{\lambda}^{\prime}, \boldsymbol{\theta}\right)\right)$, $t \geq t_{0}, \boldsymbol{\lambda}, \boldsymbol{\lambda}^{\prime} \in \Omega_{\lambda}, \boldsymbol{\theta} \in \Omega_{\theta}$, is $\left(\lambda, \lambda^{\prime}, \theta\right)$-uniformly persistently exciting, then the system is uniquely identifiable, it satisfies condition $\mathrm{A} 2$, and $\mathcal{E}_{0}(\boldsymbol{\lambda}, \boldsymbol{\theta})$ coincides with $\mathcal{E}(\boldsymbol{\lambda}, \boldsymbol{\theta})$.

We are now ready to state the main result:

Theorem 7 Consider system (7) together with the observer defined by (22), (28)-(30). Suppose that Assumptions 3.1, 3.2, and 4.1 hold. Then there exist a constant $\bar{\gamma} \in \mathbb{R}_{>0}$ and functions $r_{1}, r_{2} \in \mathcal{K}$ such that if $\gamma, \varepsilon$ are the corresponding parameters of (28), and $\gamma \in(0, \bar{\gamma})$, $\varepsilon>r_{1}\left(\Delta_{\xi}\right)$, then

$$
\lim \sup _{t \rightarrow \infty} \operatorname{dist}\left(\left(\begin{array}{c}
\hat{\boldsymbol{\lambda}}(t) \\
\hat{\boldsymbol{\theta}}(t)
\end{array}\right), \mathcal{E}(\boldsymbol{\lambda}, \boldsymbol{\theta})\right) \leq r_{2}(\varepsilon)
$$

If, in addition, $\mathcal{E}(\boldsymbol{\lambda}, \boldsymbol{\theta})$ coincides with $\mathcal{E}_{0}(\boldsymbol{\lambda}, \boldsymbol{\theta})$ then there is an $r_{3} \in \mathcal{K}$ :

$$
\limsup _{t \rightarrow \infty}\|\hat{\mathbf{x}}(t)-\mathbf{x}(t)\| \leq r_{3}(\varepsilon)
$$

The proof of Theorem 7 is presented in the next section.

Let us briefly comment on the assumptions made in the theorem statement. Assumptions 3.1, 3.2 are standard; condition A1 in Assumption 4.1 is the conventional requirement ensuring exponential convergence of $\hat{\mathbf{x}}(t), \hat{\boldsymbol{\theta}}(t)$ to $\mathbf{x}(t)$ and $\boldsymbol{\theta}$ provided that the value of $\boldsymbol{\lambda}$ and hence the values of $\boldsymbol{\varphi}(t, \boldsymbol{\lambda}, y(t))$ are known (cf. Theorem 3); A2 ensures that the distance from $(\hat{\boldsymbol{\lambda}}, \hat{\boldsymbol{\theta}})$ to the set $\mathcal{E}(\boldsymbol{\lambda}, \boldsymbol{\theta})$ is inferable from the values of $y(t)-\hat{y}(t)$ over $\left[t_{0}, \infty\right)$ (cf. Lemma 12). Note that nonlinear dependence of $\boldsymbol{\eta}$ on $\boldsymbol{\lambda}, \boldsymbol{\lambda}^{\prime}$ can impose certain technical and computational difficulties whilst checking that this condition holds. Finally, observe that the state estimation in the proposed scheme requires that $\mathcal{E}_{0}(\boldsymbol{\lambda}, \boldsymbol{\theta})=\mathcal{E}(\boldsymbol{\lambda}, \boldsymbol{\theta})$. System (15) illustrates that violation of this assumption may prevent the reconstruction of the state from observed output data.

The value of $\bar{\gamma}$ and the functions $r_{1}, r_{2}, r_{3}$ could in principle be given explicitly. However, due to dependence of these functions on $\mathbf{A}, \mathbf{B}, \mathbf{C}$, parameters $D_{\varphi}, D_{g}, M_{\varphi}$, $M_{g}$ and $T, L, \mu, \beta$ specified in Assumptions 3.1, 3.2 and Definitions 4, 5, explicit expressions for $\bar{\gamma}$ and $r_{1}, r_{2}, r_{3}$ are too lengthy and thus are removed from the theorem's statement. They are, nevertheless, provided in the proof (see e.g. (59), (69)). A procedure for finding the values of $\bar{\gamma}$ and $\varepsilon$ is discussed in Section 7 .

The value of $\varepsilon$, viz. the accuracy of the estimation, is determined by the bound $\Delta_{\xi}$ on the amplitude of perturbation $\boldsymbol{\xi}(t)$. This dependency is established through the function $r_{1}$ determining a lower bound for parameter $\varepsilon$ in (28). If no perturbation $\boldsymbol{\xi}(t)$ is present in the right-hand side of (7) then the value of $\varepsilon$ can be chosen arbitrarily small. Note that the convergence itself is asymptotic and not necessarily exponential. This is the price for the presence of unknown parameters $\boldsymbol{\lambda}$ in (7).

Remark 8 The estimate $\hat{\boldsymbol{\lambda}}(t)$ is guaranteed to converge to a single element of $\Omega_{\lambda}$ (see $(60)$ ); estimates $\hat{\boldsymbol{\theta}}(t)$ may oscillate due the influence of $\boldsymbol{\xi}(t)$. These oscillations are bounded, and will eventually be confined to the $2 r_{2}(\varepsilon)$-neighborhood of $\mathcal{E}(\boldsymbol{\lambda}, \boldsymbol{\theta})$. Hence, for $t_{1}$ sufficiently large and all $t \geq t_{1} \geq t_{0}$, the $2 r_{2}(\varepsilon)$-neighborhood of $(\hat{\boldsymbol{\lambda}}(t), \hat{\boldsymbol{\theta}}(t))$ will always contain an element of $\mathcal{E}(\boldsymbol{\lambda}, \boldsymbol{\theta})$. This element may not necessarily be from $\Omega_{\lambda} \times \Omega_{\theta}$. If the elements of $\mathcal{E}(\boldsymbol{\lambda}, \boldsymbol{\theta})$ are separated by distances exceeding $3 r_{2}(\varepsilon)$ then the estimates are guaranteed to converge to the $r_{2}(\varepsilon)$-vicinity of just one element. This point in $\mathcal{E}(\boldsymbol{\lambda}, \boldsymbol{\theta})$ will depend on $\boldsymbol{\xi}, \mathbf{x}_{0}$, and on the initial state of the observer.

Remark 9 The function $\beta$ in Definition 5 can be allowed to depend on $(\boldsymbol{\lambda}, \boldsymbol{\theta})$. In view of Remark 6 , this relaxes the requirement that $\left(\boldsymbol{\varphi}^{T}\left(t, \boldsymbol{\lambda}^{\prime}, y(t)\right), \mathbf{R}\left(t, \boldsymbol{\lambda}, \boldsymbol{\lambda}^{\prime}, \boldsymbol{\theta}\right)\right)$ in (33) (as a function of $t,\left(\boldsymbol{\lambda}, \boldsymbol{\lambda}^{\prime}, \boldsymbol{\theta}\right)$ for $t \geq t_{0}$ ) is $\left(\lambda, \lambda^{\prime}, \theta\right)$-UPE to the condition that $\left(\boldsymbol{\varphi}^{T}\left(t, \boldsymbol{\lambda}^{\prime}, y(t)\right)\right.$, 
$\left.\mathbf{R}\left(t, \boldsymbol{\lambda}, \boldsymbol{\lambda}^{\prime}, \boldsymbol{\theta}\right)\right)$ is $\lambda^{\prime}$-UPE. Note that this will make $r_{2}$ in (34) dependent on $(\boldsymbol{\lambda}, \boldsymbol{\theta})$. Finally, note that A2 need not hold for all $(\boldsymbol{\lambda}, \boldsymbol{\theta}) \in \mathbb{R}^{p+m}$ and can be restricted to the union of $\Omega_{\lambda} \times \Omega_{\theta}$ and the domain to which $(\hat{\boldsymbol{\lambda}}(t), \hat{\boldsymbol{\theta}}(t))$ belong for $t \geq t_{0}$.

\section{$5 \quad$ Proof of Theorem 7}

According to Assumption 3.2 and (8), functions $\boldsymbol{\varphi}$, $\mathbf{g}$ and $\boldsymbol{\xi}$ are continuous and are bounded in $\mathbb{R}_{\geq t_{0}} \times \Omega_{\lambda} \times \mathcal{D}_{y}$, $\mathbb{R}_{\geq t_{0}} \times \Omega_{\lambda} \times \mathcal{D}_{y} \times \mathcal{D}_{u}$ and $\mathbb{R}_{\geq t_{0}}$ respectively. Therefore solutions of the combined system, (7), (22), (28)(30) exist and are defined for all $t \geq t_{0}$. Let us denote $\mathbf{e}=\operatorname{col}\left(\mathbf{e}_{1}, \mathbf{e}_{2}\right), \mathbf{e}_{1}:=\hat{\mathbf{x}}-\mathbf{x}, \mathbf{e}_{2}:=\hat{\boldsymbol{\theta}}-\boldsymbol{\theta}, \boldsymbol{\alpha}(t, \hat{\boldsymbol{\lambda}})=$ $\boldsymbol{\varphi}(t, \hat{\boldsymbol{\lambda}}, y(t))$. Then according to (7) and (22) the following holds along the solutions of (7), (22), (28)-(30)

$$
\begin{aligned}
\left(\begin{array}{c}
\dot{\mathbf{e}}_{1} \\
\dot{\mathbf{e}}_{2}
\end{array}\right)= & \left(\begin{array}{cc}
\mathbf{A}+\boldsymbol{\ell} \mathbf{C}^{T} & \mathbf{B} \boldsymbol{\alpha}^{T}(t, \hat{\boldsymbol{\lambda}}(t)) \\
-\gamma_{\theta} \boldsymbol{\alpha}(t, \hat{\boldsymbol{\lambda}}(t)) \mathbf{C}^{T} & 0
\end{array}\right)\left(\begin{array}{l}
\mathbf{e}_{1} \\
\mathbf{e}_{2}
\end{array}\right) \\
& +\left(\begin{array}{c}
\mathbf{v}(t, \hat{\boldsymbol{\lambda}}(t), \boldsymbol{\lambda}, y(t), u(t)) \\
0
\end{array}\right)
\end{aligned}
$$

where the function $\mathbf{v}$ :

$$
\begin{aligned}
& \mathbf{v}(t, \hat{\boldsymbol{\lambda}}, \boldsymbol{\lambda}, y, u)=\mathbf{B}\left(\varphi^{T}(t, \hat{\boldsymbol{\lambda}}, y)-\boldsymbol{\varphi}^{T}(t, \boldsymbol{\lambda}, y)\right) \boldsymbol{\theta} \\
&+\mathbf{g}(t, \hat{\boldsymbol{\lambda}}, y, u)-\mathbf{g}(t, \boldsymbol{\lambda}, y, u)-\boldsymbol{\xi}(t) .
\end{aligned}
$$

is continuous and bounded for all $y \in \mathcal{D}_{y}, u \in \mathcal{D}_{u}$, $\lambda, \hat{\lambda} \in \Omega_{\lambda}$ and $t \geq t_{0}$.

The proof of the theorem is split into three parts. In the first part we consider systems

$$
\left(\begin{array}{c}
\dot{\mathbf{e}}_{1} \\
\dot{\mathbf{e}}_{2}
\end{array}\right)=\left(\begin{array}{cc}
\mathbf{A}+\boldsymbol{\ell} \mathbf{C}^{T} & \mathbf{B} \boldsymbol{\alpha}^{T}(t, \hat{\boldsymbol{\lambda}}(t)) \\
-\gamma_{\theta} \boldsymbol{\alpha}(t, \hat{\boldsymbol{\lambda}}(t)) \mathbf{C}^{T} & 0
\end{array}\right)\left(\begin{array}{c}
\mathbf{e}_{1} \\
\mathbf{e}_{2}
\end{array}\right),
$$

where $\gamma_{\theta} \in \mathbb{R}_{>0}$ and $\hat{\boldsymbol{\lambda}}: \mathbb{R}_{\geq t_{0}} \rightarrow \Omega_{\lambda}$ is a continuous, differentiable and bounded function. Let $\Phi(t)$ be a fundamental solution matrix of (38), and denote $\Phi\left(t, t_{0}\right)=$ $\Phi(t) \Phi\left(t_{0}\right)^{-1}$. Since $\Phi\left(t_{0}, t_{0}\right)$ is the identity matrix we say that $\Phi\left(t, t_{0}\right)$ is the normalized solution matrix of (38). We show that if Assumptions 3.1, 3.2 and condition A1 of Assumption 4.1 hold then there are positive numbers $M_{\lambda}, \rho$, and $D_{\rho}$ such that the fundamental (normalized) matrix of solutions, $\Phi\left(t, t_{0}\right)$, of (38) with $\hat{\boldsymbol{\lambda}}:\|\dot{\hat{\boldsymbol{\lambda}}}(t)\| \leq M_{\lambda}$ satisfies the inequality $\left\|\Phi\left(t, t_{0}\right) \mathbf{p}\right\| \leq$ $D_{\rho} e^{-\rho\left(t-t_{0}\right)}\|\mathbf{p}\|, \mathbf{p} \in \mathbb{R}^{n+m}, t \geq t_{0}$.

Using this result, in the second part of the proof we demonstrate existence of $\bar{\gamma}$ and an $\varepsilon$, dependent on $\Delta_{\xi}$, such that setting $\gamma \in(0, \bar{\gamma}]$ ensures that the estimate $\hat{\boldsymbol{\lambda}}(t)$ converges to a $\boldsymbol{\lambda}^{*}$ from $\Omega_{\lambda}$ : $\lim _{t \rightarrow \infty} \hat{\boldsymbol{\lambda}}(t)=\boldsymbol{\lambda}^{*}$.
In the third part of the proof we show that condition A2 of Assumption 4.1 guarantees that (35) holds and that, subject to the condition that $\mathcal{E}_{0}(\boldsymbol{\lambda}, \boldsymbol{\theta})$ coincides with $\mathcal{E}(\boldsymbol{\lambda}, \boldsymbol{\theta})$, property (34) holds too.

Part 1 of the proof is contained in the following.

Lemma 10 Consider system (38), and suppose that

C1) the matrices $\mathbf{A}, \mathbf{B}$, and $\mathbf{C}$ satisfy Assumption 3.1

C2) the restriction of the function $\boldsymbol{\alpha}$ in the right-hand side of (38) on $\mathbb{R}_{\geq t_{0}} \times \Omega_{\lambda}$ is $\lambda$-UPE with constants $T, \mu$ as in (31)

C3) the function $\boldsymbol{\alpha}(t, \cdot)$ is Lipschitz in $\Omega_{\boldsymbol{\lambda}}$ uniformly in $t, t \geq t_{0}$ : there is a $D \in \mathbb{R}_{>0}$ such that $\| \boldsymbol{\alpha}(t, \boldsymbol{\lambda})-$ $\boldsymbol{\alpha}\left(t, \boldsymbol{\lambda}^{\prime}\right)\|\leq D\| \boldsymbol{\lambda}-\boldsymbol{\lambda}^{\prime} \| \forall \boldsymbol{\lambda}, \boldsymbol{\lambda}^{\prime} \in \Omega_{\lambda}, t \geq t_{0}$

C4) the function $\boldsymbol{\alpha}$ and its partial derivatives wrt $t, \boldsymbol{\lambda}$ are bounded; that is there is a constant $M$ such that $\max \left\{\|\boldsymbol{\alpha}(t, \boldsymbol{\lambda})\|,\left\|\frac{\partial \boldsymbol{\alpha}(t, \boldsymbol{\lambda})}{\partial t}\right\|,\left\|\frac{\partial \boldsymbol{\alpha}(t, \boldsymbol{\lambda})}{\partial \boldsymbol{\lambda}}\right\|\right\} \leq M \forall \boldsymbol{\lambda} \in$ $\Omega_{\lambda}, \forall t \geq t_{0}$.

Then there exist $\rho, D_{\rho} \in \mathbb{R}_{>0}$ such that for all $\hat{\boldsymbol{\lambda}}: \mathbb{R}_{\geq t_{0}} \rightarrow$ $\Omega_{\lambda}, \hat{\lambda} \in \mathcal{C}^{1}$ :

$$
\begin{gathered}
\|\dot{\hat{\boldsymbol{\lambda}}}(t)\| \leq M_{\lambda} \\
0 \leq M_{\lambda} \leq \mu r /\left(2 D M T^{2}\right), r \in[0,1)
\end{gathered}
$$

the following holds

$$
\left\|\mathbf{\Phi}\left(t_{2}, t_{1}\right) \mathbf{p}\right\| \leq e^{-\rho\left(t_{2}-t_{1}\right)} D_{\rho}\|\mathbf{p}\|, \mathbf{p} \in \mathbb{R}^{n+m},
$$

where $t_{2} \geq t_{1} \geq t_{0}$, and $\mathbf{\Phi}(\cdot, \cdot)$ is the fundamental (normalized) solution matrix of (38).

The proof of Lemma 10 and other auxiliary results are provided in Appendix.

Part 2. Consider now the interconnection of (7) with the observer (22), (28)-(30). The dynamics of the combined system in the coordinates $\mathbf{e}, \hat{\boldsymbol{\lambda}}$ is described by (36), (28)(30). Recall that $\mathbf{e}(t), \hat{\boldsymbol{\lambda}}(t)$ are defined for all $t \geq t_{0}$. With respect to $\mathbf{e}(t)$, the following holds

$$
\begin{aligned}
& \mathbf{e}(t)=\Phi\left(t, t_{0}\right) \mathbf{e}\left(t_{0}\right) \\
& +\int_{t_{0}}^{t} \Phi(t, \tau)\left(\begin{array}{c}
\mathbf{v}(\tau, \hat{\boldsymbol{\lambda}}(\tau), \boldsymbol{\lambda}, y(\tau), u(\tau)) \\
0
\end{array}\right) d \tau
\end{aligned}
$$

The variable $\hat{\boldsymbol{\lambda}}$ in the combined system, as a function of $t$, is bounded, continuous, and differentiable with bounded derivative. Moreover, for any $\mathbf{A}, \mathbf{B}, \mathbf{C}, \boldsymbol{\theta} \in \Omega_{\theta}, \boldsymbol{\lambda} \in \Omega_{\lambda}$ and for any choice of $\boldsymbol{\ell}, \gamma_{\theta}$ in (22) we have that $\left|\dot{\hat{\lambda}}_{j}(t)\right| \leq$ $\gamma M_{\sigma} \max _{i}\left|\omega_{i}\right| \frac{\lambda_{i, \max }-\lambda_{i, \min }}{2}$, where $M_{\sigma}, \omega_{i}$, and $\gamma$ are defined in (27), (30), and (28). Thus $\forall t \geq t_{0}$ we have:

$$
\hat{\boldsymbol{\lambda}}(t) \in \Omega_{\lambda},\|\dot{\hat{\boldsymbol{\lambda}}}(t)\| \leq \gamma \sqrt{p} M_{\sigma} \max _{i}\left|\omega_{i}\right| \frac{\lambda_{i, \max }-\lambda_{i, \min }}{2},
$$


where $p$ is the dimension of $\boldsymbol{\lambda}$. According to Assumption 3.2, function $\boldsymbol{\alpha}$ is bounded, differentiable, and Lipschitz in the second argument. In particular, $\|\boldsymbol{\alpha}(t, \hat{\boldsymbol{\lambda}})\| \leq$ $B_{\varphi},\left\|\frac{\boldsymbol{\alpha}(t, \hat{\boldsymbol{\lambda}})}{\partial t}\right\| \leq M_{\varphi},\left\|\frac{\boldsymbol{\alpha}(t, \hat{\boldsymbol{\lambda}})}{\partial \hat{\boldsymbol{\lambda}}}\right\| \leq D_{\varphi} \forall t \geq t_{0}, \hat{\boldsymbol{\lambda}} \in \Omega_{\lambda}$. Hence there is an $M=\max \left\{B_{\varphi}, M_{\varphi}, D_{\varphi}\right\}$ such that condition C4 of Lemma 10 holds. Moreover, according to A1 in Assumption 4.1, the restriction of $\boldsymbol{\alpha}$ on $\mathbb{R}_{\geq t_{0}} \times \Omega_{\lambda}$ is $\lambda$-UPE with $T, \mu$. This, together with (43), implies that requirements $\mathrm{C} 1-\mathrm{C} 4$ of Lemma 10 are satisfied.

Let $\gamma \in\left(0, \gamma^{*}\right]$, where $\gamma^{*}$ is defined as:

$$
\begin{aligned}
\gamma^{*}=\frac{\mu r}{2 D_{\varphi} \max \left\{B_{\varphi}, M_{\varphi}, D_{\varphi}\right\} T^{2}} \times \\
\left(\sqrt{p} M_{\sigma} \max _{i}\left|\omega_{i}\right| \frac{\lambda_{i, \max }-\lambda_{i, \min }}{2}\right)^{-1} .
\end{aligned}
$$

This and (43) imply that the requirement (39) of the lemma is satisfied. Hence if $\gamma \in\left(0, \gamma^{*}\right]$ then according to Lemma 10 the matrix $\Phi\left(t, t_{0}\right)$ in (42) satisfies (41). This ensures the existence of $\rho, D_{\rho} \in \mathbb{R}_{>0}$ such that, along the solutions of (36), (28)-(30), for all $t \geq t_{0}$ we have: $\|\mathbf{e}(t)\| \leq e^{-\rho\left(t-t_{0}\right)} D_{\rho}\left\|\mathbf{e}\left(t_{0}\right)\right\|+D_{\rho} \int_{t_{0}}^{t} e^{-\rho(t-\tau)}$. $\|\mathbf{v}(\tau, \hat{\boldsymbol{\lambda}}(\tau), \boldsymbol{\lambda}, y(\tau))\| \mathrm{d} \tau$. The functions $\boldsymbol{\varphi}, \mathbf{g}$ in the definition of the function $\mathbf{v},(37)$, are Lipschitz with respect to $\boldsymbol{\lambda}$. Furthermore, according to (8) the following holds: $\|\boldsymbol{\xi}(t)\| \leq \Delta_{\xi}$. Therefore, in accordance with (37), (8), and Assumption 3.2

$$
\begin{aligned}
& \|\mathbf{v}(t, \hat{\boldsymbol{\lambda}}(t), \boldsymbol{\lambda}, y(t))\| \leq D_{v}\|\hat{\boldsymbol{\lambda}}(\tau)-\boldsymbol{\lambda}\|_{\infty,\left[t_{0}, t\right]}+\Delta_{\xi} \\
& D_{v}=D_{\varphi}\|\mathbf{B}\|\|\boldsymbol{\theta}\|+D_{g}
\end{aligned}
$$

and hence

$$
\begin{aligned}
& \|\mathbf{e}(t)\| \leq e^{-\rho\left(t-t_{0}\right)} D_{\rho}\left\|\mathbf{e}\left(t_{0}\right)\right\|+\frac{D_{\rho} D_{v}}{\rho}\|\hat{\boldsymbol{\lambda}}(\tau)-\boldsymbol{\lambda}\|_{\infty,\left[t_{0}, t\right]} \\
& +\frac{D_{\rho} \Delta_{\xi}}{\rho} .
\end{aligned}
$$

Let $j \in\{1, \ldots, p\}$, and consider the solution of

$$
\begin{aligned}
\dot{q}_{2 j-1} & =\omega_{j} \cdot\left(q_{2 j-1}-q_{2 j}-q_{2 j-1}\left(q_{2 j-1}^{2}+q_{2 j}^{2}\right)\right), \\
\dot{q}_{2 j} & =\omega_{j} \cdot\left(q_{2 j-1}+q_{2 j}-q_{2 j}\left(q_{2 j-1}^{2}+q_{2 j}^{2}\right)\right),
\end{aligned}
$$

satisfying the initial condition $q_{2 j-1}\left(t_{0}\right)=s_{2 j-1}\left(t_{0}\right)$, $q_{2 j}\left(t_{0}\right)=s_{2 j}\left(t_{0}\right)$; the parameters $\omega_{j}$ and values of $s_{2 j-1}\left(t_{0}\right), s_{2 j}\left(t_{0}\right)$ are supposed to coincide with those defined in (28), (29). The solution of (47) satisfying initial condition (29) is obviously unique, and can be expressed as a function $\mathbf{q}: \mathbb{R} \rightarrow \mathbb{R}^{2 p}$ : $q_{2 j-1}(t)=\cos \left(\omega_{j} t+\vartheta_{j}\right), \quad q_{2 j}=\sin \left(\omega_{j} t+\vartheta_{j}\right), \vartheta_{j} \in$ $\mathbb{R}, j=\{1, \ldots, p\}$. Parameters $\vartheta_{j}$ are determined in accordance with: $\cos \left(\omega_{j} t_{0}+\vartheta_{j}\right)=s_{2 j-1}\left(t_{0}\right), \sin \left(\omega_{j} t_{0}+\right.$ $\left.\vartheta_{j}\right)=s_{2 j}\left(t_{0}\right)$. Given that $\omega_{j}$ in (47) are rationallyindependent we conclude that the $\omega$-limit set of $\left(q_{1}\left(t, \mathbf{s}_{0}\right), q_{3}\left(t, \mathbf{s}_{0}\right), \ldots, q_{2 p-1}\left(t, \mathbf{s}_{0}\right)\right)$ is the hypercube $[-1,1]^{p}$ (see e.g. [20], Proposition 1.4.1). Consider the function $\boldsymbol{\beta}: \mathbb{R}^{2 p} \rightarrow \mathbb{R}^{p}$ :

$$
\beta_{j}(\mathbf{q})=\lambda_{j, \min }+\frac{\lambda_{j, \max }-\lambda_{j, \min }}{2}\left(q_{2 j-1}+1\right),
$$

and define $\overline{\boldsymbol{\lambda}}(t)=\boldsymbol{\beta}\left(\mathbf{q}\left(t, \mathbf{s}_{0}\right)\right)$. System (47), (48) satisfies conditions P1-P3, and hence we can conclude that trajectory $\overline{\boldsymbol{\lambda}}(\cdot)$ satisfies the recurrence property $(24)$ :

$$
\begin{aligned}
\forall \boldsymbol{\lambda} \in \Omega_{\lambda}, & \Delta_{\lambda} \in \mathbb{R}_{>0}, t \geq t_{0} \\
& \exists t^{\prime}>t:\left\|\boldsymbol{\lambda}-\overline{\boldsymbol{\lambda}}\left(t^{\prime}\right)\right\|<\Delta_{\lambda} .
\end{aligned}
$$

Noticing that $\mathbf{s}\left(t, \mathbf{s}_{0}\right)=\mathbf{q}\left(T(t), \mathbf{s}_{0}\right)$, where $T(t)=t_{0}+$ $\gamma \int_{t_{0}}^{t} \sigma\left(\|y(\tau)-\hat{y}(\tau)\|_{\varepsilon}\right) \mathrm{d} \tau$, we can conclude that for all $t \geq t_{0}$ the variable $\hat{\boldsymbol{\lambda}}(t)$ defined in (28) can be expressed in terms of $\overline{\boldsymbol{\lambda}}(T(t))$ as

$$
\hat{\boldsymbol{\lambda}}(t)=\overline{\boldsymbol{\lambda}}\left(t_{0}+\gamma \int_{t_{0}}^{t} \sigma\left(\|y(\tau)-\hat{y}(\tau)\|_{\varepsilon}\right) \mathrm{d} \tau\right) .
$$

Denoting $h(t)=t^{\prime}-t_{0}-\gamma \int_{t_{0}}^{t} \sigma\left(\|y(\tau)-\hat{y}(\tau)\|_{\varepsilon}\right) \mathrm{d} \tau$, where the value of $t^{\prime}$ is chosen such that (49) holds, we arrive at the following estimate:

$$
\begin{aligned}
\|\boldsymbol{\lambda}-\hat{\boldsymbol{\lambda}}(t)\| & \leq\left\|\boldsymbol{\lambda}-\overline{\boldsymbol{\lambda}}\left(t^{\prime}\right)\right\|+\left\|\overline{\boldsymbol{\lambda}}\left(t^{\prime}\right)-\hat{\boldsymbol{\lambda}}(t)\right\| \\
& =\left\|\boldsymbol{\lambda}-\overline{\boldsymbol{\lambda}}\left(t^{\prime}\right)\right\|+\left\|\overline{\boldsymbol{\lambda}}\left(t^{\prime}\right)-\overline{\boldsymbol{\lambda}}\left(t^{\prime}-h(t)\right)\right\| .
\end{aligned}
$$

The function $\overline{\boldsymbol{\lambda}}(\cdot)$ is Lipschitz: $\left\|\overline{\boldsymbol{\lambda}}\left(t_{1}\right)-\overline{\boldsymbol{\lambda}}\left(t_{2}\right)\right\| \leq$ $\sqrt{p} \max _{i} \frac{\left|\omega_{i}\right|\left(\lambda_{i, \max }-\lambda_{i, \min }\right)}{2}\left|t_{1}-t_{2}\right|, t_{1}, t_{2} \in \mathbb{R}_{\geq t_{0}}$. Thus (50), (51) imply that

$$
\begin{aligned}
& \|\boldsymbol{\lambda}-\hat{\boldsymbol{\lambda}}(t)\| \leq\left\|\boldsymbol{\lambda}-\overline{\boldsymbol{\lambda}}\left(t^{\prime}\right)\right\|+\left\|\overline{\boldsymbol{\lambda}}\left(t^{\prime}\right)-\hat{\boldsymbol{\lambda}}(t)\right\| \leq \Delta_{\lambda} \\
& +D_{\lambda}\|h(t)\|, D_{\lambda}=\sqrt{p} \max _{i} \frac{\left|\omega_{i}\right|\left(\lambda_{i, \max }-\lambda_{i, \min }\right)}{2} .
\end{aligned}
$$

Taking (46) and (52) into account we can conclude that the dynamics of the combined system (7), (22), (28)(30) obeys the following set of constraints:

$$
\begin{aligned}
\|\mathbf{e}(t)\| \leq & e^{-\rho\left(t-t_{0}\right)} D_{\rho}\left\|\mathbf{e}\left(t_{0}\right)\right\|+\frac{D_{\rho} D_{v} D_{\lambda}}{\rho}\|h(\tau)\|_{\infty,\left[t_{0}, t\right]} \\
& +\frac{D_{\rho} D_{v} \Delta_{\lambda}}{\rho}+\frac{D_{\rho} \Delta_{\xi}}{\rho} \\
h(t)= & h\left(t_{0}\right)-\gamma \int_{t_{0}}^{t} \sigma\left(\left\|\mathbf{C}^{T} \mathbf{e}_{1}(\tau)\right\|_{\varepsilon}\right) \mathrm{d} \tau .
\end{aligned}
$$

To proceed further we need an auxiliary result below.

Lemma 11 Consider a system of which the dynamics for all $t \geq t_{0}$ satisfy the following inequalities

$$
\begin{gathered}
\|\mathbf{x}(t)\| \leq \varrho\left(t-t_{0}\right)\left\|\mathbf{x}\left(t_{0}\right)\right\|+c\|h(\tau)\|_{\infty,\left[t_{0}, t\right]}+\Delta, \\
-\int_{t_{0}}^{t} \gamma_{0}\left(\|\mathbf{x}(\tau)+\mathbf{d}(\tau)\|_{\varepsilon}\right) d \tau \leq h(t)-h\left(t_{0}\right) \leq 0,
\end{gathered}
$$

where $\mathbf{x}: \mathbb{R}_{\geq t_{0}} \rightarrow \mathbb{R}^{n}, h: \mathbb{R} \rightarrow \mathbb{R}$ are trajectories reflecting the evolution of the system's state, $\mathbf{d}: \mathbb{R} \rightarrow \mathbb{R}^{n}$, 
$\|\mathbf{d}(\tau)\|_{\infty,\left[t_{0}, \infty\right)} \leq \Delta_{d}$ is a continuous and bounded function on $\left[t_{0}, \infty\right)$, @ is a strictly monotonically decreasing function with, $\varrho(0) \geq 1, \lim _{s \rightarrow \infty} \varrho(s)=0 ; c, \Delta \in \mathbb{R}_{>0}$, and $\gamma_{0}: \mathbb{R} \rightarrow \mathbb{R}_{\geq 0}$ :

$$
\left|\gamma_{0}(s)\right| \leq D_{\gamma}|s|, D_{\gamma} \in \mathbb{R}_{>0}
$$

Then $\mathbf{x}(\cdot), h(\cdot)$ in (54) are globally bounded in forward time, for $t \geq t_{0}$, provided that the following conditions hold for some $d \in(0,1), \kappa \in(1, \infty)$ :

$$
\begin{aligned}
& \varepsilon \geq \Delta\left(1+\varrho(0) \frac{\kappa}{\kappa-d}\right)+\Delta_{d}, \\
& D_{\gamma} \leq \frac{\kappa-1}{\kappa}\left[\varrho^{-1}\left(\frac{d}{\kappa}\right)\right]^{-1} \frac{h\left(t_{0}\right)}{\varrho(0)\left\|\mathbf{x}\left(t_{0}\right)\right\|+\left|h\left(t_{0}\right)\right| c\left(1+\frac{\kappa \varrho(0)}{(1-d)}\right)}
\end{aligned}
$$

The proof of Lemma 11 is provided in the Appendix. Notice that $h(t)$ in (53) satisfies $-\gamma D_{\sigma} \int_{t_{0}}^{t}\|\mathbf{e}(\tau)\|_{\varepsilon} d \tau \leq$ $h(t)-h\left(t_{0}\right) \leq 0$. Indeed, $\left\|\mathbf{C}^{T} \mathbf{e}_{1}\right\|_{\varepsilon} \leq\|\mathbf{e}\|_{\varepsilon}$ by virtue of definition of $\|\cdot\|_{\varepsilon}$ and $\mathbf{C}$, and the function $\sigma$ in (53) is Lipschitz (see (27)). Thus (53) is of the form (54) where

$$
\begin{aligned}
& c=D_{\rho} D_{v} D_{\lambda} / \rho, \Delta=D_{\rho} D_{v} \Delta_{\lambda} / \rho+D_{\rho} \Delta_{\xi} / \rho, \\
& \varrho(s)=D_{\rho} e^{-\rho s} .
\end{aligned}
$$

Notice also that because (49) holds, the value of $t^{\prime}$ in (52) can be chosen arbitrarily large. This implies that the value of $h\left(t_{0}\right)$ in (53) may be chosen arbitrarily large too. Having this in mind, and invoking Lemmas 10, 11 we can conclude that choosing $\varepsilon, \gamma$ in (28) as

$$
\begin{aligned}
& \varepsilon \geq r_{0}(\Delta), r_{0}(\Delta)=\Delta\left(1+D_{\rho} \frac{\kappa}{\kappa-d}\right), \\
& 0<\gamma<\bar{\gamma}=\min \left\{\gamma^{*}, D_{\gamma, \infty}\right\} \\
& D_{\gamma, \infty}=\frac{\kappa-1}{D_{\sigma} \kappa}\left[\ln \left(D_{\rho} \frac{\kappa}{d}\right)\right]^{-1} \frac{\rho}{c\left(1+\kappa D_{\rho} /(1-d)\right)},
\end{aligned}
$$

where $\gamma^{*}$ is defined as in (44), ensures that the function $h(\cdot)$ in (53) is bounded. Given that $h(\cdot)$ by construction is monotone and bounded, the Bolzano-Weierstrass theorem implies that $h(t)$ converges to a limit, and hence

$$
\begin{aligned}
& \lim _{t \rightarrow \infty} \int_{t_{0}}^{t} \sigma\left(\left\|\mathbf{C}^{T} \mathbf{e}_{1}(\tau)\right\|_{\varepsilon}\right) d \tau=\bar{h}, \bar{h} \in \mathbb{R}, \\
& \lim _{t \rightarrow \infty} \hat{\boldsymbol{\lambda}}(t)=\boldsymbol{\lambda}^{*}, \boldsymbol{\lambda}^{*} \in \Omega_{\lambda} .
\end{aligned}
$$

Noticing that $\sigma\left(\left\|\mathbf{C}^{T} \mathbf{e}(\tau)\right\|_{\varepsilon}\right)$ is uniformly continuous and using Barbalat's lemma we conclude that

$$
\lim _{t \rightarrow \infty} \sup _{\tau \in[t, \infty)}\left\|\mathbf{C}^{T} \mathbf{e}_{1}(\tau)\right\| \leq \varepsilon
$$

Part 3. Let us rewrite the equation for $\dot{\mathbf{e}}_{1}$ in (36) as:

$$
\begin{aligned}
\dot{\mathbf{e}}_{1} & =\left(\mathbf{A}+\boldsymbol{\ell} \mathbf{C}^{T}\right) \mathbf{e}_{1}+\mathbf{v}_{1}(t, \hat{\boldsymbol{\theta}}(t), \hat{\boldsymbol{\lambda}}(t), \boldsymbol{\theta}, \boldsymbol{\lambda}) \\
& +\mathbf{v}_{2}(t, \hat{\boldsymbol{\lambda}}(t), \boldsymbol{\lambda})+\mathbf{v}_{3}(t),
\end{aligned}
$$

where $\mathbf{v}_{3}(t)=-\boldsymbol{\xi}(t)$ and

$$
\begin{aligned}
& \mathbf{v}_{1}(t, \hat{\boldsymbol{\theta}}, \hat{\boldsymbol{\lambda}}, \boldsymbol{\theta}, \boldsymbol{\lambda})=\mathbf{B}\left(\boldsymbol{\varphi}^{T}(t, \hat{\boldsymbol{\lambda}}, y(t)) \hat{\boldsymbol{\theta}}-\boldsymbol{\varphi}^{T}(t, \boldsymbol{\lambda}, y(t)) \boldsymbol{\theta}\right) \\
& \mathbf{v}_{2}(t, \hat{\boldsymbol{\lambda}}, \boldsymbol{\lambda})=\mathbf{g}(t, \hat{\boldsymbol{\lambda}}, y(t), u(t))-\mathbf{g}(t, \boldsymbol{\lambda}, y(t), u(t)) .
\end{aligned}
$$

Next steps make use of the following lemma.

\section{Lemma 12 Consider}

$$
\begin{aligned}
& \dot{\mathbf{x}}=\mathbf{A} \mathbf{x}+\mathbf{u}(t)+\mathbf{d}(t), \\
& y=\mathbf{C}^{T} \mathbf{x}, \mathbf{x}\left(t_{0}\right)=\mathbf{x}_{0}, \mathbf{x}_{0} \in \mathbb{R}^{n},
\end{aligned}
$$

where $\mathbf{A}$ and $\mathbf{C}$ are defined as in (1), and $\mathbf{u}, \mathbf{d}$ : $\mathbb{R} \rightarrow \mathbb{R}^{n}, \mathbf{u} \in \mathcal{C}^{1}, \mathbf{d} \in \mathcal{C}$. Let $\mathbf{u}, \dot{\mathbf{u}}, \mathbf{d}$ be bounded: $\max \{\|\mathbf{u}(t)\|,\|\dot{\mathbf{u}}(t)\|\} \leq B,\|\mathbf{d}(t)\| \leq \Delta_{\xi}$ for all $t \geq t_{0}$.

Then, if the solution of (64) is globally bounded for all $t \geq t_{0}$, there exist $\kappa_{1}, \kappa_{2} \in \mathcal{K}$ :

$$
\begin{aligned}
& \|y(\tau)\|_{\infty,\left[t_{0}, \infty\right)} \leq \varepsilon \Rightarrow \exists t^{\prime}\left(\varepsilon, \mathbf{x}_{0}\right) \geq t_{0}: \\
& \left\|z_{1}(\tau)+u_{1}(\tau)\right\|_{\infty,\left[t^{\prime}, \infty\right)} \leq \kappa_{1}(\varepsilon)+\kappa_{2}\left(\Delta_{\xi}\right),
\end{aligned}
$$

where $z_{1}=\left(\begin{array}{llll}1 & 0 & \ldots & 0\end{array}\right) \mathbf{z}$,

$$
\begin{aligned}
\dot{\mathbf{z}} & =\Lambda \mathbf{z}+\mathbf{G u}(t), \Lambda=\left(-\mathbf{b} \mid \begin{array}{c}
I_{n-2} \\
0
\end{array}\right), \\
\mathbf{G} & =\left(\begin{array}{ll}
-\mathbf{b} I_{n-1}
\end{array}\right), \mathbf{z}\left(t_{0}\right)=0,
\end{aligned}
$$

and $\mathbf{b}=\operatorname{col}\left(b_{1}, \ldots, b_{n-1}\right)$ : real parts of the roots of $s^{n-1}+b_{1} s^{n-2}+\cdots+b_{n-1}$ are negative.

Moreover, if $\mathbf{d}(t) \equiv 0$, then

$$
\begin{aligned}
& y(t)=0 \forall t \geq t_{0} \Rightarrow \exists \mathbf{p} \in \mathbb{R}^{n-1}: \forall t \geq t_{0} \\
& \left(\begin{array}{llll}
1 & 0 & \ldots & 0
\end{array}\right) e^{\Lambda\left(t-t_{0}\right)} \mathbf{p}+z_{1}(t)+u_{1}(t)=0 .
\end{aligned}
$$

The proof of Lemma 12 is provided in the Appendix. According to (8) and Assumption 3.2, $\mathbf{v}_{1}(\cdot, \hat{\boldsymbol{\theta}}(\cdot), \hat{\boldsymbol{\lambda}}(\cdot), \boldsymbol{\theta}, \boldsymbol{\lambda})$, $\mathbf{v}_{2}(\cdot, \hat{\boldsymbol{\lambda}}(\cdot), \boldsymbol{\lambda}), \mathbf{v}_{3}(\cdot)$ and $\dot{\mathbf{v}}_{1}, \dot{\mathbf{v}}_{2}$ are bounded. Thus assumptions of Lemma 12 are met for equations (62), (63), and hence (61) implies that there is a $t_{1}(\varepsilon) \geq t_{0}$ and $\kappa_{1}, \kappa_{2} \in \mathcal{K}$ such that $\forall t \geq t_{1}(\varepsilon)$ we have:

$$
\begin{aligned}
& \| \boldsymbol{\varphi}^{T}(t, \hat{\boldsymbol{\lambda}}(t), y(t)) \hat{\boldsymbol{\theta}}(t)-\boldsymbol{\varphi}^{T}(t, \boldsymbol{\lambda}, y(t)) \boldsymbol{\theta}+v_{2,1}(t, \hat{\boldsymbol{\lambda}}(t), \boldsymbol{\lambda}) \\
& +\tilde{\mathbf{C}}^{T} \int_{t_{0}}^{t} e^{\Lambda(t-\tau)} \mathbf{G}_{2}(\tau, \hat{\boldsymbol{\lambda}}(\tau), \boldsymbol{\lambda}) d \tau \| \leq \kappa_{1}(\varepsilon)+\kappa_{2}\left(\Delta_{\xi}\right),
\end{aligned}
$$

where $\mathbf{G} \in \mathbb{R}^{(n-1) \times n}, \tilde{\mathbf{C}} \in \mathbb{R}^{n-1}$ are defined as in (18)-(20), and $v_{2,1}(\cdot)$ is the first component of $\mathbf{v}_{2}(\cdot)$. Given that $\int_{t_{0}}^{t} e^{\Lambda(t-\tau)} \mathbf{G v}_{2}(\tau, \hat{\boldsymbol{\lambda}}(\tau), \boldsymbol{\lambda}) d \tau=$ $\int_{t_{0}}^{t} e^{\Lambda(t-\tau)} \mathbf{G v}_{2}\left(\tau, \boldsymbol{\lambda}^{*}, \boldsymbol{\lambda}\right) d \tau+\int_{t_{0}}^{t} e^{\Lambda(t-\tau)} \mathbf{G}\left(\mathbf{v}_{2}(\tau, \hat{\boldsymbol{\lambda}}(\tau), \boldsymbol{\lambda})\right.$ 
$\left.-\mathbf{v}_{2}\left(\tau, \boldsymbol{\lambda}^{*}, \boldsymbol{\lambda}\right)\right) d \tau$, noticing that $\Lambda$ is Hurwitz and that, according to $(60) \mathbf{v}_{2}(t, \hat{\boldsymbol{\lambda}}(t), \boldsymbol{\lambda})-\mathbf{v}_{2}\left(t, \boldsymbol{\lambda}^{*}, \boldsymbol{\lambda}\right) \rightarrow 0$ as $t \rightarrow \infty$, we can conclude that there is a $t_{2}(\varepsilon) \geq t_{1}(\varepsilon)$ such that $\boldsymbol{\eta}\left(t, \hat{\boldsymbol{\theta}}(t), \boldsymbol{\lambda}^{*}, \boldsymbol{\theta}, \boldsymbol{\lambda}\right)$ defined as in (19) satisfies:

$$
\begin{aligned}
& \left\|\boldsymbol{\eta}\left(t, \hat{\boldsymbol{\theta}}(t), \boldsymbol{\lambda}^{*}, \boldsymbol{\theta}, \boldsymbol{\lambda}\right)\right\|=\| \boldsymbol{\varphi}^{T}\left(t, \boldsymbol{\lambda}^{*}, y(t)\right) \hat{\boldsymbol{\theta}}(t)- \\
& \boldsymbol{\varphi}^{T}(t, \boldsymbol{\lambda}, y(t)) \boldsymbol{\theta}+\tilde{\mathbf{C}}^{T} \int_{t_{0}}^{t} e^{\Lambda(t-\tau)} \mathbf{G v}_{2}\left(\tau, \boldsymbol{\lambda}^{*}, \boldsymbol{\lambda}\right) d \tau \\
& +v_{2,1}\left(t, \boldsymbol{\lambda}^{*}, \boldsymbol{\lambda}\right) \| \leq \kappa_{1}(\varepsilon)+\kappa_{2}\left(\Delta_{\xi}\right)+\varepsilon \forall t \geq t_{2}(\varepsilon) .(67)
\end{aligned}
$$

Recall that the restriction of $\boldsymbol{\alpha}_{2}\left(t,\left(\boldsymbol{\lambda}^{\prime}, \boldsymbol{\theta}^{\prime}\right),(\boldsymbol{\lambda}, \boldsymbol{\theta})\right)=$ $\boldsymbol{\eta}\left(t, \boldsymbol{\theta}^{\prime}, \boldsymbol{\lambda}^{\prime}, \boldsymbol{\theta}, \boldsymbol{\lambda}\right)$ on $\mathbb{R}_{\geq t_{0}} \times \mathbb{R}^{p+m} \times \mathbb{R}^{p+m}$ is wNPE with $L, \beta, \mathcal{E}$. Let $t_{3}(\varepsilon)$ be such that $\left\|\mathbf{C}^{T} \mathbf{e}_{1}(t)\right\|<2 \varepsilon$ for all $t \geq t_{3}(\varepsilon)$ (existence of such $t_{3}(\varepsilon)$ follows from $(61)$ ). Consider the sequence $\left\{\tau_{i}\right\}_{i=0}^{\infty}, \tau_{i}=\max \left\{t_{3}(\varepsilon), t_{2}(\varepsilon)\right\}+i L$. Since $\boldsymbol{\varphi}(\cdot, \hat{\boldsymbol{\lambda}}(\cdot), y(\cdot))$ is bounded, there is an $M_{\theta} \in \mathbb{R}_{>0}$ :

$$
\left\|\hat{\boldsymbol{\theta}}(\tau)-\hat{\boldsymbol{\theta}}\left(\tau_{i}\right)\right\|_{\infty,\left[t_{i}, t_{i+1}\right]} \leq \varepsilon 2 \gamma_{\theta} B_{\varphi} L=\varepsilon M_{\theta}
$$

for all $t \geq \tau_{0}$. Hence $\forall t: t \in\left[\tau_{i}, \tau_{i+1}\right], i \in \mathbb{N}$, we have: $\left\|\boldsymbol{\eta}\left(t, \hat{\boldsymbol{\theta}}\left(\tau_{i}\right), \boldsymbol{\lambda}^{*}, \boldsymbol{\theta}, \boldsymbol{\lambda}\right)\right\| \leq \kappa_{1}(\varepsilon)+\kappa_{2}\left(\Delta_{\xi}\right)+\varepsilon\left(M_{\theta} B_{\varphi}+1\right)$. This, however, implies that there is an $N \in \mathbb{N}$ such that dist $\left(\left(\begin{array}{c}\lambda^{*} \\ \hat{\boldsymbol{\theta}}\left(\tau_{i}\right)\end{array}\right), \mathcal{E}(\boldsymbol{\lambda}, \boldsymbol{\theta})\right) \leq \beta^{-1}\left(\kappa_{1}(\varepsilon)+\kappa_{2}\left(\Delta_{\xi}\right)+\right.$ $\left.\varepsilon\left(M_{\theta} B_{\varphi}+1\right)\right)$ for all $i \geq N$. Therefore, taking (43), (68) into account, we can conclude that there is a $t^{\prime}(\varepsilon)$ :

$$
\begin{aligned}
& \operatorname{dist}\left(\left(\begin{array}{l}
\hat{\boldsymbol{\lambda}}(t) \\
\hat{\boldsymbol{\theta}}(t)
\end{array}\right), \mathcal{E}(\boldsymbol{\lambda}, \boldsymbol{\theta})\right) \leq 2 \varepsilon M_{\theta}+ \\
& \beta^{-1}\left(\kappa_{1}(\varepsilon)+\kappa_{2}\left(\Delta_{\xi}\right)+\varepsilon\left(M_{\theta} B_{\varphi}+1\right)\right) \forall t \geq t^{\prime}(\varepsilon) .
\end{aligned}
$$

Notice that $r_{0}$ in (59) is a class $\mathcal{K}_{\infty}$ function of $\Delta$. Parameter $\Delta$, as defined in (58), is the sum: $\Delta=\frac{D_{\rho} D_{v} \Delta_{\lambda}}{\rho}+$ $\frac{D_{\rho} \Delta_{\xi}}{\rho}$. Given that the value of $\Delta_{\lambda}$ can be chosen arbitrarily, we pick $\Delta_{\lambda}=\Delta_{\xi}$. This renders $r_{0}$ in (59) a class $\mathcal{K}_{\infty}$ (and hence class $\mathcal{K}$ ) function of $\Delta_{\xi}$. Denote this function as $r_{1}$, then $\varepsilon>r_{1}\left(\Delta_{\xi}\right)$ implies that

$$
\begin{aligned}
& \beta^{-1}\left(\kappa_{1}(\varepsilon)+\kappa_{2}\left(\Delta_{\xi}\right)+\varepsilon\left(M_{\theta} B_{\varphi}+1\right)\right)+2 \varepsilon M_{\theta}< \\
& \beta^{-1}\left(\kappa_{1}(\varepsilon)+\kappa_{2}\left(r_{1}^{-1}(\varepsilon)\right)+\varepsilon\left(M_{\theta} B_{\varphi}+1\right)\right) \\
& +2 \varepsilon M_{\theta}=r_{2}(\varepsilon) .
\end{aligned}
$$

Thus (34) holds.

Finally, if $\mathcal{E}(\boldsymbol{\lambda}, \boldsymbol{\theta})$ coincides with $\mathcal{E}_{0}(\boldsymbol{\lambda}, \boldsymbol{\theta})$, then Assumption 3.2 and (34) imply that $\| \mathbf{v}_{1}(t, \hat{\boldsymbol{\theta}}(t), \hat{\boldsymbol{\lambda}}(t), \boldsymbol{\theta}, \boldsymbol{\lambda})+$ $\mathbf{v}_{2}(t, \hat{\boldsymbol{\lambda}}(t), \boldsymbol{\lambda}) \|<M_{1} r_{2}(\varepsilon)$ for some $M_{1} \in \mathbb{R}_{>0}, t \geq t^{\prime}(\varepsilon)$. Since $\mathbf{A}+\boldsymbol{\ell} \mathbf{C}^{T}$ in (62) is Hurwitz, (35) follows.

\section{Discussion and generalization}

\subsection{Removing passivity requirement (Assumption 3.1)}

Theorem 7 requires that $\mathbf{A}, \mathbf{B}, \mathbf{C}$ in (7) satisfy Assumption 3.1. Here we invoke the idea of filtered transformations [26], [27] to show how observer (22), (28) can be modified so that this condition could be replaced with the requirement that the pair $\mathbf{A}, \mathbf{C}$ is observable. Consider a generalization of (7)

$$
\begin{aligned}
& \dot{\mathbf{x}}=\mathbf{A} \mathbf{x}+\mathbf{\Psi}(t, \boldsymbol{\lambda}, y) \boldsymbol{\theta}+\mathbf{g}(t, \boldsymbol{\lambda}, y, u(t))+\boldsymbol{\xi}(t), \\
& y=\mathbf{C}^{T} \mathbf{x}, \mathbf{A}=\left(\begin{array}{cc}
0 & \mathbf{I}_{n-1} \\
0 & 0
\end{array}\right), \mathbf{C}=\operatorname{col}(1,0, \ldots, 0),
\end{aligned}
$$

where $\boldsymbol{\Psi}: \mathbb{R} \times \mathbb{R}^{p} \times \mathbb{R} \rightarrow \mathbb{R}^{n \times m}, \boldsymbol{\Psi} \in \mathcal{C}^{1}$, is Lipschitz in $\boldsymbol{\lambda}$, and $\boldsymbol{\Psi}(\cdot, \boldsymbol{\lambda}, y(\cdot)), \dot{\boldsymbol{\Psi}}(\cdot, \boldsymbol{\lambda}, y(\cdot))$ are bounded on $\mathbb{R}_{\geq t_{0}}$. The function $\boldsymbol{\xi}$ and parameters are defined as in (7), and the function $\mathbf{g}$ satisfies Assumption 3.2.

Let $\mathbf{B}=\operatorname{col}\left(1, b_{1}, \ldots, b_{n-1}\right)$ be a vector such that the polynomial $s^{n-1}+b_{1} s^{n-2}+\cdots+b_{n-1}$ is Hurwitz. As an observer candidate for (70) we propose a system in which $\mathcal{S}_{w}$ is defined as in (28), and $\mathcal{S}_{a}$ is given as follows:

$$
\begin{gathered}
\dot{\mathbf{M}}=\left(\mathbf{A}-\mathbf{B C}^{T} \mathbf{A}\right) \mathbf{M}+\left(\mathbf{I}_{n}-\mathbf{B C}^{T}\right) \mathbf{\Psi}(t, \hat{\boldsymbol{\lambda}}(t), y(t)), \\
\dot{\hat{\boldsymbol{\zeta}}}=\mathbf{A} \hat{\boldsymbol{\zeta}}+\boldsymbol{\ell}\left(\mathbf{C}^{T} \hat{\boldsymbol{\zeta}}-y(t)\right)+\mathbf{B} \boldsymbol{\varphi}^{T}(t, \hat{\boldsymbol{\lambda}}(t), y(t),[\hat{\boldsymbol{\lambda}}, y]) \hat{\boldsymbol{\theta}} \\
\quad+\mathbf{g}(t, \hat{\boldsymbol{\lambda}}(t), y(t), u(t)), \quad \\
\dot{\hat{\boldsymbol{\theta}}}=-\gamma_{\theta}\left(\mathbf{C}^{T} \hat{\boldsymbol{\zeta}}-y(t)\right) \boldsymbol{\varphi}(t, \hat{\boldsymbol{\lambda}}(t), y(t),[\hat{\boldsymbol{\lambda}}, y]), \gamma_{\theta} \in \mathbb{R}_{>0}, \\
\hat{\mathbf{x}}=\hat{\boldsymbol{\zeta}}+\mathbf{M} \hat{\boldsymbol{\theta}}, \quad \mathbf{M} \in \mathbb{R}^{n \times m}, \quad \mathbf{M}\left(t_{0}\right)=0,
\end{gathered}
$$

where

$$
\begin{aligned}
\varphi^{T}(t, \hat{\boldsymbol{\lambda}}(t), y(t),[\hat{\boldsymbol{\lambda}}, y])= & \mathbf{C}^{T} \mathbf{A} \mathbf{M}(t,[\hat{\boldsymbol{\lambda}}, y]) \\
& +\mathbf{C}^{T} \mathbf{\Psi}(t, \hat{\boldsymbol{\lambda}}(t), y(t)) .
\end{aligned}
$$

The first row of $\mathbf{M}$ is zero for all $t \geq t_{0}$, and $\hat{y}=\mathbf{C}^{T} \hat{\mathbf{x}}=$ $\mathbf{C}^{T} \hat{\boldsymbol{\zeta}}$. Since $\boldsymbol{\Psi}(\cdot, \hat{\boldsymbol{\lambda}}(\cdot), y(\cdot))$ is bounded on $\mathbb{R}_{\geq t_{0}}$ and Lipschitz in $\hat{\boldsymbol{\lambda}}, \mathbf{M}(\cdot,[\hat{\boldsymbol{\lambda}}, y]), \mathbf{M}(\cdot,[\hat{\boldsymbol{\lambda}}, y])$ are globally bounded on $\mathbb{R}_{\geq t_{0}}$, and $\mathbf{M}(t,[\hat{\boldsymbol{\lambda}}, y])$ is Lipschitz in $\hat{\boldsymbol{\lambda}}$ for $\hat{\boldsymbol{\lambda}}=$ const. Let $\boldsymbol{\zeta}=\mathbf{x}-\mathbf{M} \boldsymbol{\theta}$, then using (70)-(72) we can write

$$
\begin{array}{r}
\dot{\boldsymbol{\zeta}}=\mathbf{A} \boldsymbol{\zeta}+\mathbf{B} \boldsymbol{\varphi}(t, \hat{\boldsymbol{\lambda}}(t), y(t),[\hat{\boldsymbol{\lambda}}, y]) \boldsymbol{\theta}+(\boldsymbol{\Psi}(t, \boldsymbol{\lambda}, y(t), u(t)) \\
-\boldsymbol{\Psi}(t, \hat{\boldsymbol{\lambda}}(t), y(t), u(t))) \boldsymbol{\theta}+\mathbf{g}(t, \boldsymbol{\lambda}, y(t), u(t))+\boldsymbol{\xi}(t) .
\end{array}
$$

Dynamics of (70), (71) in the coordinates $\mathbf{e}_{1}=\hat{\boldsymbol{\zeta}}-\mathbf{x}+$ 
$\mathbf{M} \boldsymbol{\theta}, \mathbf{e}_{2}=\hat{\boldsymbol{\theta}}-\boldsymbol{\theta}$ is

$$
\begin{aligned}
\left(\begin{array}{l}
\dot{\mathbf{e}}_{1} \\
\dot{\mathbf{e}}_{2}
\end{array}\right)= & \left(\begin{array}{cc}
\mathbf{A}+\boldsymbol{\ell} \mathbf{C}^{T} & \mathbf{B} \boldsymbol{\alpha}^{T}(t) \\
-\gamma_{\theta} \boldsymbol{\alpha}(t) \mathbf{C}^{T} & 0
\end{array}\right)\left(\begin{array}{l}
\mathbf{e}_{1} \\
\mathbf{e}_{2}
\end{array}\right) \\
& +\left(\begin{array}{c}
\tilde{\mathbf{v}}(t, \hat{\boldsymbol{\lambda}}(t), \boldsymbol{\lambda}, y(t), u(t)) \\
0
\end{array}\right)
\end{aligned}
$$

where $\boldsymbol{\alpha}(t)=\boldsymbol{\varphi}(t, \hat{\boldsymbol{\lambda}}(t), y(t),[\hat{\boldsymbol{\lambda}}, y]), \tilde{\mathbf{v}}(t, \hat{\boldsymbol{\lambda}}, \boldsymbol{\lambda}, y, u)=$ $(\boldsymbol{\Psi}(t, \hat{\boldsymbol{\lambda}}, y)-\boldsymbol{\Psi}(t, \boldsymbol{\lambda}, y)) \boldsymbol{\theta}+\mathbf{g}(t, \hat{\boldsymbol{\lambda}}, y, u)-\mathbf{g}(t, \boldsymbol{\lambda}, y, u)-$ $\boldsymbol{\xi}(t)$. Since the pair $\mathbf{A}, \mathbf{C}$ is observable there always is an $\boldsymbol{\ell}$ so that (2) holds. The structure of (73) is now identical to that of (36), and Assumptions 3.1, 3.2 hold for the functions $\boldsymbol{\varphi}, \mathrm{g}$ in (71). Finally, consider the function $\boldsymbol{\eta}_{1}$ :

$\boldsymbol{\eta}_{1}\left(t, \boldsymbol{\lambda}, \boldsymbol{\theta}, \boldsymbol{\lambda}^{\prime}, \boldsymbol{\theta}^{\prime}\right)=\boldsymbol{\varphi}^{T}\left(t, \boldsymbol{\lambda}^{\prime}, y(t),\left[\boldsymbol{\lambda}^{\prime}, y\right]\right)\left(\boldsymbol{\theta}^{\prime}-\boldsymbol{\theta}\right)+$ $\mathbf{C}^{T}\left(\boldsymbol{\Psi}\left(t, \boldsymbol{\lambda}^{\prime}, y(t)\right)-\boldsymbol{\Psi}(t, \boldsymbol{\lambda}, y(t))\right) \boldsymbol{\theta}+g_{1}\left(t, \boldsymbol{\lambda}^{\prime}, y(t), u(t)\right)-$ $g_{1}(t, \boldsymbol{\lambda}, y(t), u(t))+q\left(t, \boldsymbol{\lambda}^{\prime}, \boldsymbol{\lambda}, \boldsymbol{\theta}\right)$,

where $q\left(t, \boldsymbol{\lambda}^{\prime}, \boldsymbol{\lambda}, \boldsymbol{\theta}\right)=\tilde{\mathbf{C}} \mathbf{z}\left(t, \boldsymbol{\lambda}, \boldsymbol{\lambda}^{\prime}, \boldsymbol{\theta}\right), \dot{\mathbf{z}}=\Lambda \mathbf{z}+$ $\mathbf{G}\left(\left(\Psi\left(t, \boldsymbol{\lambda}^{\prime}, y(t)\right)-\Psi(t, \boldsymbol{\lambda}, y(t))\right) \boldsymbol{\theta}+\mathbf{g}\left(t, \boldsymbol{\lambda}^{\prime}, y(t), u(t)\right)-\right.$ $\mathbf{g}(t, \boldsymbol{\lambda}, y(t), u(t))), \mathbf{z}\left(t_{0}\right)=0$, and $\tilde{\mathbf{C}}, \Lambda, \mathbf{G}$ are defined as in (20). The following is now immediate:

Theorem 13 Consider (70), (71), (28)-(30). Suppose that condition A1 of Assumption 4.1 holds for the function $\boldsymbol{\alpha}_{3}: \mathbb{R}_{>t_{0}} \times \Omega_{\lambda} \rightarrow \mathbb{R}^{m}, \boldsymbol{\alpha}_{3}(t, \boldsymbol{\lambda})=$ $\boldsymbol{\varphi}(t, \boldsymbol{\lambda}, y(t),[\boldsymbol{\lambda}, y])$, where $\boldsymbol{\varphi}$ is defined as in (71). Furthermore, let the restriction of $\boldsymbol{\alpha}_{4}: \mathbb{R} \times \mathbb{R}^{p+m} \times$ $\mathbb{R}^{p+m} \rightarrow \mathbb{R}, \boldsymbol{\alpha}_{4}\left(t,(\boldsymbol{\lambda}, \boldsymbol{\theta}),\left(\boldsymbol{\lambda}^{\prime}, \boldsymbol{\theta}^{\prime}\right)\right)=\boldsymbol{\eta}_{1}\left(t, \boldsymbol{\lambda}, \boldsymbol{\theta}, \boldsymbol{\lambda}^{\prime}, \boldsymbol{\theta}^{\prime}\right)$ on $\mathbb{R}_{\geq t_{0}} \times \mathbb{R}^{p+m} \times \mathbb{R}^{p+m}$ be weakly nonlinearly persistently exciting in $(\boldsymbol{\lambda}, \boldsymbol{\theta})$ wrt to the map $\mathcal{E}_{1}$ :

$$
\begin{aligned}
& \mathcal{E}_{1}(\boldsymbol{\lambda}, \boldsymbol{\theta})=\left\{\left(\boldsymbol{\lambda}^{\prime}, \boldsymbol{\theta}^{\prime}\right), \boldsymbol{\lambda}^{\prime} \in \mathbb{R}^{p}, \boldsymbol{\theta}^{\prime} \in \mathbb{R}^{m} \mid \mathbf{B}\left(\boldsymbol{\theta}^{\prime}-\boldsymbol{\theta}\right)^{T} .\right. \\
& \boldsymbol{\varphi}\left(t, \boldsymbol{\lambda}^{\prime}, y(t),\left[\boldsymbol{\lambda}^{\prime}, y\right]\right)+\left(\boldsymbol{\Psi}\left(t, \boldsymbol{\lambda}^{\prime}, y(t)\right)-\boldsymbol{\Psi}(t, \boldsymbol{\lambda}, y(t))\right) \boldsymbol{\theta} \\
& \left.+\mathbf{g}\left(t, \boldsymbol{\lambda}^{\prime}, y(t), u(t)\right)-\mathbf{g}(t, \boldsymbol{\lambda}, y(t), u(t))=0, \forall t \geq t_{0}\right\} .
\end{aligned}
$$

Then there exist a constant $\bar{\gamma} \in \mathbb{R}_{>0}$ and functions $r_{1}, r_{2}, r_{3} \in \mathcal{K}$ such that if $\gamma, \varepsilon$ are the corresponding parameters of (28), and $\gamma \in(0, \bar{\gamma}), \varepsilon>r_{1}\left(\Delta_{\xi}\right)$, then (35), (34) hold (with $\mathcal{E}$ replaced by $\mathcal{E}_{1}$ ) for the interconnection (70), (71), and (28).

The proof is largely identical to that of Theorem 7 (a sketch is presented in the Appendix). According to Remarks 6,9 one can replace the requirement that the restriction of $\boldsymbol{\alpha}_{4}$ on $\mathbb{R}_{>t_{0}} \times \mathbb{R}^{p+m} \times \mathbb{R}^{p+m}$ is wNPE with $L, \beta, \mathcal{E}_{1}$ with that of the $\lambda^{\prime}$-uniform persistency of excitation of the the restriction of $\boldsymbol{\alpha}_{5}$ :

$$
\boldsymbol{\alpha}_{5}\left(t, \boldsymbol{\lambda}^{\prime}\right)=\left(\boldsymbol{\varphi}^{T}\left(t, \boldsymbol{\lambda}^{\prime}, y(t),\left[\boldsymbol{\lambda}^{\prime}, y\right]\right), \mathbf{R}_{1}\left(t, \boldsymbol{\lambda}, \boldsymbol{\lambda}^{\prime}, \boldsymbol{\theta}\right)\right),
$$

where $\mathbf{R}_{1}\left(t, \boldsymbol{\lambda}, \boldsymbol{\lambda}^{\prime}, \boldsymbol{\theta}\right)=\int_{0}^{1} \frac{\partial}{\partial \mathbf{s}} r_{1}\left(t, \mathbf{s}\left(\xi, \boldsymbol{\lambda}, \boldsymbol{\lambda}^{\prime}\right), \boldsymbol{\theta}\right) d \xi$, $\mathbf{s}\left(\xi, \boldsymbol{\lambda}, \boldsymbol{\lambda}^{\prime}\right)=\boldsymbol{\lambda}^{\prime} \xi+(1-\xi) \boldsymbol{\lambda}$, and $r_{1}(t, \boldsymbol{\lambda}, \boldsymbol{\theta})=\mathbf{C}^{T}$
$\boldsymbol{\Psi}(t, \boldsymbol{\lambda}, y(t)) \boldsymbol{\theta}+g_{1}(t, \boldsymbol{\lambda}, y(t), u(t))+\tilde{\mathbf{C}}^{T} \int_{t_{0}}^{t} e^{\Lambda(t-\tau)} \mathbf{G}$ $(\boldsymbol{\Psi}(\tau, \boldsymbol{\lambda}, y(\tau)) \boldsymbol{\theta}+\mathbf{g}(\tau, \boldsymbol{\lambda}, y(\tau), u(\tau))) d \tau$, on $\mathbb{R}_{\geq t_{0}} \times \Omega_{\lambda}$.

Consider now systems (9). Since $\mathbf{A}, \mathbf{C}$ is observable, there is a coordinate transformation $\mathbf{x} \mapsto T(\mathbf{A}) \mathbf{x}$ bringing system (9) into the form (70), albeit with the functions $\boldsymbol{\Psi}, \mathbf{g}$ and vector $\boldsymbol{\theta}$ defined differently. An example illustrating the viability of this approach is provided in Section 7. Notice also that observability of $\mathbf{A}, \mathbf{C}$ implies that the system $\dot{\mathbf{x}}=\mathbf{A} \mathbf{x}+\tilde{\mathbf{\Psi}}(t, \boldsymbol{\lambda}, \mathbf{x}) \boldsymbol{\theta}+\tilde{\mathbf{g}}(t, \boldsymbol{\lambda}, \mathbf{x}, u(t))+$ $\boldsymbol{\xi}(t), y=\mathbf{C}^{T} \mathbf{x}$, in which the functions $\tilde{\boldsymbol{\Psi}}, \tilde{\mathbf{g}}$ are bounded and Lipschitz in $\mathbf{x}$ can be brought into the form (70) by using an auxiliary high-gain observer (cf. [16]).

\subsection{Presence of measurement noise}

Suppose now that observations of system (7) output, $y$, are corrupted by noise. That is, instead of $y=\mathbf{C}^{T} \mathbf{x}$ we can access only the variable $y_{d}=\mathbf{C}^{T} \mathbf{x}+d, y_{d} \in \mathcal{D}_{y}$, where $d: \mathbb{R} \rightarrow \mathbb{R}, d \in \mathcal{C}^{1},\|d(\tau)\|_{\infty,\left[t_{0}, \infty\right)} \leq \Delta_{d}, \Delta_{d} \in$ $\mathbb{R}_{\geq 0}$, and $|\dot{d}(t)|$ is bounded. In this case the variable $y$ in the observer definition (22), (28) is replaced by $y_{d}$, and the dynamics of $\mathbf{e}_{1}=\hat{\mathbf{x}}-\mathbf{x}, \mathbf{e}_{2}=\hat{\boldsymbol{\theta}}-\boldsymbol{\theta}$ becomes:

$$
\begin{aligned}
\left(\begin{array}{l}
\dot{\mathbf{e}}_{1} \\
\dot{\mathbf{e}}_{2}
\end{array}\right)= & \left(\begin{array}{cc}
\mathbf{A}+\boldsymbol{\ell} \mathbf{C}^{T} & \mathbf{B} \boldsymbol{\alpha}^{T}(t, \hat{\boldsymbol{\lambda}}(t)) \\
-\gamma_{\theta} \boldsymbol{\alpha}(t, \hat{\boldsymbol{\lambda}}(t)) \mathbf{C}^{T} & 0
\end{array}\right)\left(\begin{array}{l}
\mathbf{e}_{1} \\
\mathbf{e}_{2}
\end{array}\right) \\
& +\left(\begin{array}{c}
\mathbf{v}\left(t, \hat{\boldsymbol{\lambda}}(t), \boldsymbol{\lambda}, y_{d}(t), u(t)\right) \\
0
\end{array}\right)+\left(\begin{array}{l}
\boldsymbol{\xi}_{1}(t) \\
\boldsymbol{\xi}_{2}(t)
\end{array}\right)
\end{aligned}
$$

where $\boldsymbol{\alpha}^{T}(t, \hat{\boldsymbol{\lambda}})=\varphi^{T}\left(t, \hat{\boldsymbol{\lambda}}, y_{d}(t)\right), \mathbf{v}\left(t, \hat{\boldsymbol{\lambda}}, \boldsymbol{\lambda}, y_{d}, u\right)=$ $\mathbf{B}\left(\varphi^{T}\left(t, \hat{\boldsymbol{\lambda}}, y_{d}\right)-\boldsymbol{\varphi}^{T}\left(t, \boldsymbol{\lambda}, y_{d}\right)\right) \boldsymbol{\theta}+\mathbf{g}\left(t, \hat{\boldsymbol{\lambda}}, y_{d}, u\right)-$ $\mathbf{g}\left(t, \boldsymbol{\lambda}, y_{d}, u\right)-\boldsymbol{\xi}(t)$, and $\boldsymbol{\xi}_{1}(t)=\mathbf{B}\left(\boldsymbol{\varphi}^{T}\left(t, \boldsymbol{\lambda}, y_{d}(t)\right)-\right.$ $\left.\boldsymbol{\varphi}^{T}(t, \boldsymbol{\lambda}, y(t))\right) \boldsymbol{\theta}+\left(\mathbf{g}\left(t, \boldsymbol{\lambda}, y_{d}(t), u(t)\right)-\mathbf{g}(t, \boldsymbol{\lambda}, y(t), u(t))\right)-$ $\boldsymbol{\ell} d(t), \boldsymbol{\xi}_{2}(t)=-\gamma_{\theta} d(t) \boldsymbol{\alpha}(t, \hat{\boldsymbol{\lambda}}(t))$. It can now be seen that if $\boldsymbol{\varphi}, \mathbf{g}$ are Lipschitz in $y$ then there is an $M_{d}>0$ such that $\max \left\{\left\|\boldsymbol{\xi}_{1}(\tau)\right\|_{\infty,\left[t_{0}, \infty\right)},\left\|\boldsymbol{\xi}_{2}(\tau)\right\|_{\infty,\left[t_{0}, \infty\right)}\right\} \leq M_{d} \Delta_{d}$. Thus invoking Lemmas 10, 11 and following the argument provided in proof of Theorem 7 one can establish existence of $\gamma>0$ and $\varepsilon>0$ such that (60), (61) hold. Convergence of the estimates will also follow subject to corresponding persistency of excitation requirements (cf. part 3 of the proof). An illustration of the influence of measurement noise on performance of the observer is provided in Section 7, Fig. 2.

\section{Examples}

Consider the following system:

$$
\begin{aligned}
& \dot{\mathbf{x}}=\mathbf{A} \mathbf{x}+\mathbf{B} \theta+\mathbf{B}\left(\sin (\lambda \cos (t))+e^{\lambda \sin (t)}\right)+\boldsymbol{\xi}(t), \\
& \mathbf{A}=\left(\begin{array}{ll}
-2 & 1 \\
-1 & 0
\end{array}\right), \mathbf{B}=\left(\begin{array}{l}
1 \\
1
\end{array}\right), \begin{array}{l}
y=\mathbf{C}^{T} \mathbf{x}, \mathbf{x}\left(t_{0}\right)=\mathbf{x}_{0}, \\
\mathbf{C}=\operatorname{col}(1,0, \ldots, 0),
\end{array}
\end{aligned}
$$


where $\theta \in[0,1]=\Omega_{\theta}, \lambda \in[0.1,1]=\Omega_{\lambda}$ are unknown parameters, and $\mathbf{x}_{0}$ is only partially known. The function $\boldsymbol{\xi}: \mathbb{R} \rightarrow \mathbb{R}^{2}, \boldsymbol{\xi}(t)=0.001 \operatorname{col}(\sin (t), \cos (t))$, stands for the unmodeled dynamics and is supposed to be unavailable for direct observation.

Let the task be to infer the values of $\mathbf{x}, \theta, \lambda$ from the measurements of $y$ over time. System (75) belongs to the class of equations described by $(7)$ with $\varphi(t, \lambda, y)=1$ $\forall t, \lambda, y$, and $\mathbf{g}(t, \lambda, y, u)=\mathbf{B}\left(\sin (\lambda \cos (t))+e^{\lambda \sin (t)}\right)$. Moreover, it satisfies Assumption 3.1 with

$$
\mathbf{P}=\left(\begin{array}{cc}
2 & -1 \\
-1 & 1
\end{array}\right), \mathbf{Q}=\left(\begin{array}{cc}
6 & -3 \\
-3 & 2
\end{array}\right), \boldsymbol{\ell}=\left(\begin{array}{l}
0 \\
0
\end{array}\right)
$$

and Assumption 3.2 with $D_{\varphi}=0, D_{g}=B_{g}=M_{g}=$ $\sqrt{2}(1+e), B_{\varphi}=1$, and $M_{\varphi}=0$. According to $(22)$ (28) the observer candidate is:

$$
\begin{aligned}
& \dot{\hat{\mathbf{x}}}=\mathbf{A} \hat{\mathbf{x}}+\mathbf{B} \hat{\theta}+\mathbf{B}\left(\sin (\hat{\lambda} \cos (t))+e^{\hat{\lambda} \sin (t)}\right) \\
& \dot{\hat{\theta}}=-\gamma_{\theta}\left(\mathbf{C}^{T} \hat{\mathbf{x}}-y(t)\right) \\
& \dot{s}_{1}=\gamma \tanh \left(\left\|\mathbf{C}^{T} \hat{\mathbf{x}}-y(t)\right\|_{\varepsilon}\right)\left(s_{1}-s_{2}-s_{1}\left(s_{1}^{2}+s_{2}^{2}\right)\right) \\
& \dot{s}_{2}=\gamma \tanh \left(\left\|\mathbf{C}^{T} \hat{\mathbf{x}}-y(t)\right\|_{\varepsilon}\right)\left(s_{1}+s_{2}-s_{2}\left(s_{1}^{2}+s_{2}^{2}\right)\right) \\
& \hat{\lambda}=0.1+0.45\left(s_{1}+1\right), s_{1}^{2}\left(t_{0}\right)+s_{2}^{2}\left(t_{0}\right)=1
\end{aligned}
$$

Parameters $\gamma, \gamma_{\theta}$, and $\varepsilon$ will be specified in a later stage.

Note that $\boldsymbol{\varphi}(t, \lambda, y)=1$. Hence condition A1 in Assumption 4.1 holds. Notice also that the function $\boldsymbol{\eta}$, as defined in (19), in this case becomes: $\boldsymbol{\eta}\left(t, \lambda, \theta, \lambda^{\prime}, \theta^{\prime}\right)=$ $\theta-\theta^{\prime}+\sin (\lambda \cos (t))+e^{\lambda \sin (t)}-\sin \left(\lambda^{\prime} \cos (t)\right)-e^{\lambda^{\prime} \sin (t)}$. One can now check that condition A2 is satisfied as well. According to Theorem 7, system (76), (77) is an adaptive observer for (75) subject to the choice of $\gamma, \gamma_{\theta}$, and $\varepsilon$. A procedure for setting specific values of these parameters can be derived from the argument provided in the proof of the theorem. Let us show how this procedure works in this example.

According to the theorem, parameter $\gamma_{\theta}$ is an arbitrary positive number; here, for simplicity, we set $\gamma_{\theta}=1$. Parameters $\gamma, \varepsilon$ are to satisfy (58), (59). The choice of $\gamma$ is subjected to two constraints. The first constraint is $\gamma \in$ $\left(0, \gamma^{*}\right]$, where $\gamma^{*}$ is specified in (44). It ensures that the restriction of $\boldsymbol{\varphi}(\cdot, \hat{\lambda}(\cdot), y(\cdot))$ on $\mathbb{R}_{\geq t_{0}}$ is persistently exciting. In our case $\varphi(t, \hat{\lambda}(t), y(t))$ is independent on $\hat{\lambda}(t)$, and this property holds for any $\gamma>0$. The second constraint is: $\gamma<\frac{\kappa-1}{D_{\sigma} \kappa}\left[\ln \left(D_{\rho} \frac{\kappa}{d}\right)\right]^{-1} \frac{\rho}{c\left(1+\kappa D_{\rho} /(1-d)\right)}, \kappa>$ $1, d \in(0,1), D_{\sigma}=1, c=D_{\rho} D_{v} D_{\lambda} / \rho$, where $D_{\lambda}=$ $0.45, D_{v}=\sqrt{2}(1+e)$ (see $\left.(52),(45)\right)$, and $\rho$ and $D_{\rho}$ are such that the fundamental matrix of solutions of (38), $\Phi\left(t, t_{0}\right)$, satisfies: $\left\|\Phi\left(t, t_{0}\right) \mathbf{p}\right\| \leq D_{\rho} e^{-\rho\left(t-t_{0}\right)}\|\mathbf{p}\|$. In this example

$$
\Phi\left(t, t_{0}\right)=e^{\mathbf{A}_{1}\left(t-t_{0}\right)}, \mathbf{A}_{1}=\left(\begin{array}{rrr}
-2 & 1 & 1 \\
-1 & 0 & 1 \\
-1 & 0 & 0
\end{array}\right)
$$

and $\rho=0.5, D_{\rho}=4.242$. Picking $d=0.2, \kappa=2$ results in $\bar{\gamma}=0.00286$. Since the values of $d, \kappa$ are now defined, we can set the value of $\varepsilon$. Taking (58), (59) into account and noticing that $\Delta_{\lambda}=0$ (this is because trajectories of (77) in which the term $\tanh \left(\left\|\mathbf{C}^{T} \hat{\mathbf{x}}-y(t)\right\|_{\varepsilon}\right)$ is replaced with 1 will pass through every point of $\left.\Omega_{\lambda}=[0.1,1]\right)$, we arrive at $\varepsilon \geq \frac{D_{\rho} \Delta_{\xi}}{\rho}\left(1+D_{\rho} \frac{\kappa}{\kappa-d}\right)$, where $\Delta_{\xi}:\|\boldsymbol{\xi}(t)\| \leq$ $\Delta_{\xi}$. Given that $\Delta_{\xi}=0.001$ we obtain: $\varepsilon \geq 0.018$.

Computer simulation results of the combined system (75) - (77) with parameters $\theta=0.2, \lambda=0.7, \gamma=0.0028$, $\gamma_{\theta}=1, \varepsilon=0.018$ are summarized in Fig. 2. As can be observed, the system has two weakly attracting sets (marked as white circles). These sets correspond to the true values of $\theta$ and $\lambda$. Even though trajectories of the system are converging to the attracting sets asymptotically, small neighborhoods of these sets are not forwardinvariant. Hence the sets themselves are not globally asymptotically stable, albeit they are clearly attracting. Middle panel depicts typical trajectories of $\hat{\lambda}$ and $\hat{\theta}$. To show how the proposed observer behaves in presence of measurement noise we simulated the model-observer system in which signal $y(t)=x_{1}(t)$ in the observer subsystem was replaced with $y_{d}(t)=x_{1}(t)+0.05 \sin (2 t)$. The value of $\varepsilon$ was changed to 0.068 to account for this perturbation. The observer retained functionality, albeit with lower precision of estimation.

In order to illustrate the behavior of the system in the case of multiple equivalent parameterizations, we simulated a modified version of the combined system (75)-(77), in which the nonlinearly parameterized terms, i.e. $\mathbf{B}\left(\sin (\lambda \cos (t))+e^{\lambda \sin (t)}\right)$ in $(75)$ and $\mathbf{B}\left(\sin (\hat{\lambda} \cos (t))+e^{\hat{\lambda} \sin (t)}\right)$ in $(76)$, are replaced with $\mathbf{B}\left(\sin \left((\lambda-0.45)^{2} \cos (t)\right)+e^{(\lambda-0.45)^{2} \sin (t)}\right)$ and $\mathbf{B}\left(\sin \left((\hat{\lambda}-0.45)^{2} \cos (t)\right)+e^{(\hat{\lambda}-0.45)^{2} \sin (t)}\right)$ respectively. Assumptions 3.1, 3.2 and A1 in Assumption 4.1 still hold for the modified system (with the same values of parameters). Yet, system (75) with the modified $\mathbf{g}(t, \lambda)=\mathbf{B}\left(\sin \left((\lambda-0.45)^{2} \cos (t)\right)+e^{(\lambda-0.45)^{2} \sin (t)}\right)$ is no longer uniquely identifiable since $\mathbf{g}(t, 0.7)=\mathbf{g}(t, 0.2)$ for all $t$. Simulation results of the modified system are presented in Fig, 2, right panel. Instead of two attracting sets as in the previous configuration, the modified system has four weakly attracting sets corresponding to two equivalent parameterizations $\theta=0.2, \lambda=0.7$ (true) and $\theta=0.2, \lambda=0.2$ (spurious). Parameter estimates converge to small vicinities of these alternative parameterizations. Note that the estimates do not jump 

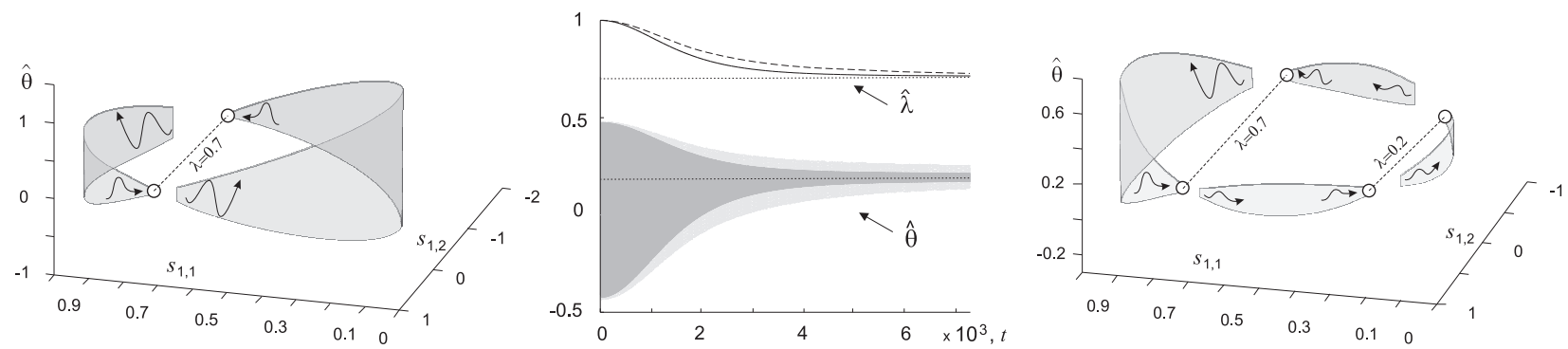

Figure 2. Left panel: a qualitative picture of the system dynamics in the coordinates $\left(s_{1}, s_{2}, \hat{\theta}\right)$. Shaded regions depict envelops of 15 trajectories of the system for various initial conditions. Actual trajectories of the system are very oscillatory, and individual trajectories are hardly distinguishable. Qualitatively, their behavior is shown by the black arrowed lines. Middle panel: typical simulated trajectories of $\hat{\theta}, \hat{\lambda}$ as functions of $t$. Solid and dark grey curves correspond to the case when no measurement noise is added; dashed and light grey curves show trajectories of the estimates in presence of additive measurement noise. Dotted lines indicate true values of $\theta$ and $\lambda$. Right panel: envelops of the modified system solutions in the coordinates $\left(s_{1}, s_{2}, \hat{\theta}\right)$.

between neighborhoods of $\theta=0.2, \lambda=0.7$ and $\theta=0.2$, $\lambda=0.2$, which is consistent with Remark 8 .

Finally, we illustrate the applicability of our approach to models (9). Consider the third example from Table 1 with nominal parameter values as follows: $\tau_{m}=0.1666$, $\tau_{s}=5, A_{f}=1, \sigma_{f}=2, \sigma_{s}=0.8$. Suppose that true values of these parameters are unknown, but it is known that they are within $\pm 25 \%$ of their nominal values. Since the pair $\mathbf{A}, \mathbf{C}$ is observable, there is a parameterdependent coordinate transformation $\mathbf{x} \mapsto T \mathbf{x}$, $T=\left(\begin{array}{cc}1 & 0 \\ \tau_{s}^{-1} & -\tau_{m}^{-1}\end{array}\right)$ rendering the original equations into (70) with $\boldsymbol{\Psi}(t, \lambda, y)=\left(\begin{array}{cccc}y & \tanh (\lambda y) & 0 & 0 \\ 0 & 0 & y & \tanh (\lambda y)\end{array}\right)$, $\mathbf{g}=0$ and $\boldsymbol{\theta}=\operatorname{col}\left(-\frac{1}{\tau_{s}}-\frac{1}{\tau_{m}}, \frac{A_{f}}{\tau_{m}},-\frac{1+\sigma_{s}}{\tau_{m} \tau_{s}}, \frac{A_{f}}{\tau_{m} \tau_{s}}\right)$, $\lambda=\frac{\sigma_{f}}{A_{f}}$. Note that $\mathbf{A}, \boldsymbol{\Psi}$ and $\boldsymbol{\theta}$ differ from those in the original parametrizaton. Let $\mathbf{B}=\operatorname{col}(1,1)$ and consider $\mathbf{M}(t,[\lambda, y])=\left(m_{i j}(t,[\lambda, y])\right), i=1,2, j=1, \ldots, 4$ in (71). It is clear that the polynomial $s+1$ formed by the coefficients of $\mathbf{B}$ is Hurwitz, $m_{1, j}(t,[\lambda, y])=0$, $m_{2, j}(t,[\lambda, y])$ are defined as $\dot{m}_{2,1}=-\dot{m}_{2,1}-y(t)$, $\dot{m}_{2,2}=-\dot{m}_{2,2}-\tanh (\lambda y(t)), \dot{m}_{2,3}=-\dot{m}_{2,3}+y(t)$, $\dot{m}_{2,4}=-\dot{m}_{2,2}+\tanh (\lambda y(t)), m_{2, j}\left(t_{0}\right)=0$, and that $\boldsymbol{\varphi}(t,[\lambda, y])=\operatorname{col}\left(m_{2,1}(t,[y])+y(t), m_{2,2}(t,[\lambda, y])+\right.$ $\left.\tanh (\lambda y(t)), m_{2,3}(t,[y]), m_{2,4}(t,[\lambda, y])\right)$. Given that $A_{f}$, $\sigma_{f}$ vary within $25 \%$ of their nominal values we obtain that $\Omega_{\lambda}=[1.2,3.33]$. For the given system, $y$ is bounded, $\boldsymbol{\varphi}(t,[\lambda, y])$, as a function of $t, \boldsymbol{\lambda}$ on $\mathbb{R}_{\geq t_{0}} \times \Omega_{\lambda}$, is $\lambda$-UPE with $T=100, \mu=0.08$. Moreover the restriction of $\boldsymbol{\alpha}_{5}$, defined in (74), on $\mathbb{R}_{\geq t_{0}} \times \Omega_{\lambda}$ is $\lambda^{\prime}$-UPE with $T=100, \mu=0.0054$. Hence assumptions of Theorem 13 are met. We simulated the system and observer (71), (28) with $\gamma_{\theta}=4, \gamma=0.004$, and $\varepsilon=0$ for various initial conditions and values of $\boldsymbol{\theta}, \lambda ; \hat{\boldsymbol{\theta}}, \hat{\lambda}$ approached true values of $\boldsymbol{\theta}, \lambda$ asymptotically as prescribed. An example of typical behavior of the estimates is shown in Fig. 3. Original parameters of the model can be recov-

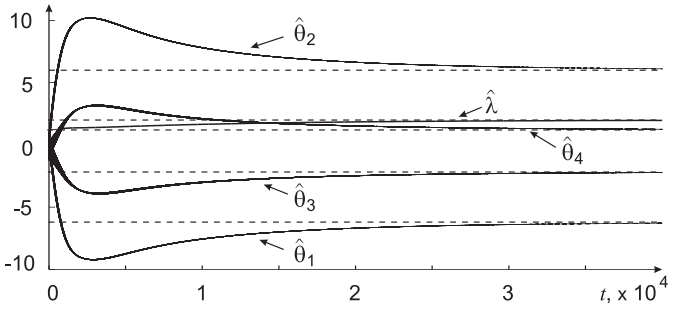

Figure 3. Estimates $\hat{\boldsymbol{\theta}}, \hat{\lambda}$ as functions of $t$. True values of $\boldsymbol{\theta}$, $\lambda$ are shown as dashed lines.

ered as: $\hat{\tau}_{s}=\hat{\theta}_{2} / \hat{\theta}_{4}, \hat{\tau}_{m}=-1 /\left(\hat{\theta}_{1}+1 / \hat{\tau}_{s}\right), \hat{A}_{f}=\hat{\tau}_{m} \hat{\theta}_{2}$, $\hat{\sigma}_{s}=-\hat{\theta}_{3} \hat{\tau}_{s} \hat{\tau}_{m}-1, \hat{\sigma}_{f}=\hat{A}_{f} \hat{\lambda}$. Further examples may be found in the supplementary material [38].

\section{Conclusion}

We derived an observer that can reconstruct asymptotically the unknown state and parameter values of a class of systems with general nonlinear parametrization. This class can be viewed as an extension of the adaptive observer canonical forms [5], [25]. In contrast to earlier approaches addressing the problem of nonlinear parametrization in the problem of adaptive observer design [12],[15], [14], [16], [22], [39], the class of parameterizations for which the reconstruction is guaranteed is not limited to convex/concave or one-to-one functions. We showed that reconstruction can be achieved, subject to additional conditions of linear/nonlinear persistency of excitation, if nonlinearly parameterized functions in the model are bounded, differentiable and Lipschitz.

The set to which the estimates converge is not guaranteed to be asymptotically stable. Yet the set is attracting in a weaker, Milnor sense, cf. [31]. Numerical simulations revealed that the convergence time in our approach depends heavily on the dimension of $\boldsymbol{\lambda}$; it does not, however, depend crucially on the dimension of $\boldsymbol{\theta}$. This renders the method more efficient than exhaustive search; the smaller the dimension of $\boldsymbol{\lambda}$ the more advantageous our method becomes. In this respect a related 
question arises: is there a "best" parametrization for a given physical model in the class of systems (7) or (9)? The answer is likely to require quantitative assessment of the performance of various observers for all admissible parametrizations. We do not answer this question here, but hope to be able to address it in future.

\section{References}

[1] H.D.I. Abarbanel, D. Creveling, R. Farisian, and M. Kostuk. Dynamical state and parameter estimation. SIAM J. Applied Dynamical Systems, 8(4):1341-1381, 2009.

[2] A. Alessandri, M. Baglietto, and G. Battistelli. Movinghorizon state estimation for nonlinear discrete-time systems: New stability results and approximation schemes. Automatica, 44:1753-1765, 2008.

[3] V.I. Arnold. Mathematical Methods in Classical Mechanics. Springer-Verlag, 1978.

[4] G. Bastin and D. Dochain. On-line Estimation and Adaptive Control of Bioreactors. Elsevier, 1990.

[5] G. Bastin and M. Gevers. Stable adaptive observers for nonlinear time-varying systems. IEEE Trans. on Automatic Control, 33(7):650-658, 1988.

[6] G. Besancon. Remarks on nonlinear adaptive observer design. Systems and Control Letters, 41:271-280, 2000.

[7] J.D. Boskovic. Stable adaptive control of a class of first-order nonlinearly parameterized plants. IEEE Trans. on Automatic Control, 40(2):347-350, 1995.

[8] C. Cao, A.M. Annaswamy, and A. Kojic. Parameter convergence in nonlinearly parametrized systems. IEEE Trans. on Automatic Control, 48(3):397-411, 2003.

[9] M. Chapell. Structural identifiability of models characterizing saturable binding: Comparison of pseudo-steady-state and non-pseudo-steady state model formulations. Mathematical Biosciences, 133:1-20, 1996.

[10] L. Denis-Vidal and Joly-Blanchard. Equivalence and identifiability analysis of uncontrolled nonlinear dynamical systems. Automatica, 40:287-292, 2004.

[11] J. Distefano and C. Cobelli. On parameter and structural identifiabiliy: Nonunique observability/reconstructibility for identifiable systems, other ambiguities, and new definitions. IEEE Trans. on Automatic Control, AC-25(4):830-833, 1980.

[12] M. Farza, M. M'Saad, T. Maatoung, and M. Kamoun. Adaptive observers for nonlinearly parameterized class of nonlinear systems. Automatica, 45:2292-2299, 2009.

[13] A. Gorban and I. Karlin. Invariant Manifolds for Physical and Chemical Kinetics. Lecture Notes in Physics, Springer, 2005.

[14] H. Grip. State and parameter estimation for linear systems with nonlinearly parameterized perturbations. In Proceedings of the 48-th IEEE Conference on Decision and Control, pages 8218-8225, 2009.

[15] H.F. Grip, T.A. Johansen, L. Imsland, and G.O. Kaasa. Parameter estimation and compensation in systems with nonlinearly parameterized perturbations. Automatica, 46(1):19-28, 2010.

[16] H.F. Grip, A. Saberi, and T.A. Johansen. Estimation of states and parameters for linear systems with nonlinearly parameterized perturbations. Systems and Control Letters, 60(9):771-777, 2011.
[17] A. Ilchmann. Universal adaptive stabilization of nonlinear systems. Dynamics and Control, (7):199-213, 1997.

[18] E. Izhikevich. Dynamical Systems in Neuroscience: The Geometry of Excitability and Bursting. MIT Press, 2007.

[19] T. Johnson and W. Tucker. Rigorous parameter reconstruction for differential equations with noisy data. Automatica, 44:2422-2426, 2008.

[20] A. Katok and B. Hasselblatt. Introduction to the modern theory of dynamical systems. Cambridge Univ. Press, 1999.

[21] Z. Lin and C. Knospe. A saturated high-gain contol for a benchmark experiment. In Proceedings of the American Control Conference, pages 2644-2648, 2000.

[22] X. Liu, R. Ortega, H. Su, and J. Chu. On adaptive control of nonlinearly parameterized nonlinear systems: Towards a constructive procedure. Systems Control Letters, 60:36-43, 2011.

[23] A. Loria. Explicit convergence rates for MRAC-type systems. Automatica, 40(8):1465-1468, 2004.

[24] A. Loria and E. Panteley. Uniform exponential stability of linear time-varying systems: revisited. Systems and Control Letters, 47(1):13-24, 2003.

[25] R. Marino. Adaptive observers for single output nonlinear systems. IEEE Trans. on Automatic Control, 35(9):10541058, 1990.

[26] R. Marino and P. Tomei. Global adaptive observers for nonlinear systems via filtered transformations. IEEE Trans. on Automatic Control, 37(8):1239-1245, 1992.

[27] R. Marino and P. Tomei. Global adaptive output-feedback control of nonlinear systems, part I: Linear parameterization. IEEE Trans. on Automatic Control, 38(1):17-32, 1993.

[28] R. Marino and P. Tomei. Global adaptive outputfeedback control of nonlinear systems, part II: Nonlinear parameterization. IEEE Trans. on Automatic Control, 38(1):33-48, 1993.

[29] B. Martensson. The order of any stabilizing regulator is sufficient a priori information for adaptive stabilization. Systems and Control Letters, 6(2):87-91, 1985.

[30] B. Martensson and J.W. Polderman. Correction and simplification to "the order of any stabilizing regulator is sufficient a priori information for adaptive stabilization". Systems and Control Letters, 20(6):465-470, 1993.

[31] J. Milnor. On the concept of attractor. Commun. Math. Phys., 99:177-195, 1985.

[32] S. Moreau and J.-C. Trigeassou. Modelling and identification of a non-linear saturated magnetic circuit: Theoretical study and experimental results. Mathematics and Computers in Simulation, 71:446-459, 2006.

[33] V.V. Nemytskii and V.V. Stepanov. Qualitative theory of differential equations. Princeton Univ. Press, 1960.

[34] Jean-Baptiste Pomet. Remarks on sufficient information for adaptive nonlinear regulation. In Proceedings of the 31-st IEEE Conference on Decision and Control, pages 1737-1739, 1992.

[35] A. Poyton, M. Varziri, K. McAuley, P. McLellan, and J. Ramsey. Parameter estimation in continuous-time dynamic models using principal differential analysis. Computers and Chemical Engineering, 30:698-708, 2006.

[36] C.V. Rao, J.B. Rawlings, and D.Q. Mayne. Constrained state estimation for nonlinear discrete-time systems: Stability and moving horizon approximation. IEEE Trans. on Automatic Control, 48:246-258, 2003. 
[37] P. Rowat and A. Selverston. Modeling the gastric mill central pattern generator with a relaxation-oscillator network. Journal of Neurophysiology, 70(3):1030-1053, 1993.

[38] I. Tyukin, E. Steur, H. Nijmeijer, and C. van Leeuwen. Supplementary material for: Adaptive observers and parameter estimation for a class of systems nonlinear in the parameters. http://arxiv.org/abs/1304.4020, 2013.

[39] I.Yu. Tyukin, D. V. Prokhorov, and C. van Leeuwen. Adaptation and parameter estimation in systems with unstable target dynamics and nonlinear parametrization. IEEE Trans. on Automatic Control, 52(9):1543-1559, 2007.

[40] I.Yu. Tyukin, E. Steur, H. Nijmeijer, and C. van Leeuwen. Non-uniform small-gain theorems for systems with unstable invariant sets. SIAM Journal on Control and Optimization, 47(2):849-882, 2008.

\section{A Appendix}

Lemma 14 Consider $\dot{y}=k y+u(t)+d(t), k \in \mathbb{R}, u, d$ : $\mathbb{R}_{\geq t_{0}} \rightarrow \mathbb{R}, u \in \mathcal{C}^{1}, d \in \mathcal{C}^{0}$, and let $\max \{|u(t)|,|\dot{u}(t)|\} \leq$ $B,|d(t)| \leq \Delta_{\xi}$. Then $\|y\|_{\infty,\left[t_{0}, \infty\right)} \leq \varepsilon \Rightarrow \exists t_{1}(\varepsilon) \geq t_{0}:$ $\|u\|_{\infty,\left[t_{1}(\varepsilon), \infty\right)} \leq \sqrt{\varepsilon}\left(1+e^{|k| \sqrt{\varepsilon}}+B\right)+\Delta_{\xi}$.

Proof of Lemma 14. Noticing that $y(t)$ for $t \geq t_{0}+T$, $T>0$, can be expressed as: $y(t)=y(t-\bar{T}) e^{k T}+$ $\int_{t-T}^{t} e^{k(t-\tau)}(u(\tau)+d(\tau)) d \tau$ and using the Meanvalue theorem we obtain: $y(t)-y(t-T) e^{k T}=$ $T e^{k\left(t-\tau^{\prime}\right)}\left(u\left(\tau^{\prime}\right)+d\left(\tau^{\prime}\right)\right), \quad \tau^{\prime} \in[t-T, t]$. Hence $\varepsilon\left(1+e^{k T}\right) \geq T e^{k\left(t-\tau^{\prime}\right)}\left(|u(t)|-T B-\Delta_{\xi}\right)$, and $\Delta_{\xi}+T B+$ $\frac{\varepsilon\left(1+e^{k T}\right)}{T \min \left\{1, e^{k T}\right\}} \geq \Delta_{\xi}+T B+\frac{\varepsilon\left(1+e^{k T}\right)}{T \min \left\{1, e^{k\left(t-\tau^{\prime}\right)}\right\}} \geq|u(t)|$ for all $t \geq t_{0}+T$. Given that $T$ can be chosen arbitrarily we let $T=\sqrt{\varepsilon}$, and thus $|u(t)| \leq \sqrt{\varepsilon}\left(1+e^{k \sqrt{\varepsilon}}\right) \max \left\{1, e^{-k \sqrt{\varepsilon}}\right\}+$ $B \sqrt{\varepsilon}+\Delta_{\xi} \leq \sqrt{\varepsilon}\left(1+e^{|k| \sqrt{\varepsilon}}+B\right)+\Delta_{\xi} \forall t \geq t_{0}+\sqrt{\varepsilon}$.

Proof of Lemma 12. Let us rewrite (64) as

$$
\begin{aligned}
& \dot{y}=a_{1} y+\tilde{\mathbf{C}} \tilde{\mathbf{x}}+u_{1}(t)+d_{1}(t) \\
& \dot{\tilde{\mathbf{x}}}=\tilde{\mathbf{A}} \tilde{\mathbf{x}}+\tilde{\mathbf{a}} y+\mathbf{b} u_{1}(t)+\mathbf{G u}(t)+\tilde{\mathbf{d}}(t),
\end{aligned}
$$

where $\tilde{\mathbf{a}}=\operatorname{col}\left(a_{2}, \ldots, a_{n}\right), \tilde{\mathbf{C}}=\operatorname{col}(1,0, \ldots, 0)$, $\tilde{\mathbf{d}}(t)=\operatorname{col}\left(d_{2}(t), \ldots, d_{n}(t)\right)$, and $\mathbf{G}=\left(-\mathbf{b} I_{n-1}\right)$, $\tilde{\mathbf{A}}=\left(\begin{array}{cc}0 & I_{n-2} \\ 0 & 0\end{array}\right)$. Let $\|y(t)\|_{\infty,\left[t_{0}, \infty\right)} \leq \varepsilon$ and denote $e(t)=\tilde{\mathbf{C}}^{T} \tilde{\mathbf{x}}(t)+u_{1}(t)$. According to Lemma 14 , there is a $t_{1}(\varepsilon)>t_{0}$ and $v_{1}, v_{2} \in \mathcal{K}$ such that $\|e(t)\|=\left\|\tilde{\mathbf{C}}^{T} \tilde{\mathbf{x}}(t)+u_{1}(t)\right\| \leq v_{1}(\varepsilon)+v_{2}\left(\Delta_{\xi}\right) \forall t \geq t_{1}(\varepsilon)$.

Using the notation above we obtain: $\dot{\tilde{\mathbf{x}}}=(\tilde{\mathbf{A}}-$ $\left.\mathbf{b} \tilde{\mathbf{C}}^{T}\right) \tilde{\mathbf{x}}+\tilde{\mathbf{a}} y(t)+\tilde{\mathbf{G}} \mathbf{u}(t)+\mathbf{b} e(t)+\tilde{\mathbf{d}}(t)$. Matrix $\tilde{\mathbf{A}}-\mathbf{b} \tilde{\mathbf{C}}^{T}=\Lambda$ is Hurwitz, and hence there are $D, k \in \mathbb{R}_{>0}$ such that $\left\|e^{\Lambda\left(t-t_{0}\right)}\right\| \leq D e^{-k\left(t-t_{0}\right)}$. Therefore $\left\|\tilde{\mathbf{C}}^{T} \tilde{\mathbf{x}}(t)-\tilde{\mathbf{C}}^{T} \int_{t_{0}}^{t} e^{\Lambda(t-\tau)} \mathbf{G u}(\tau) d \tau\right\| \leq$ $D e^{-k\left(t-t_{0}\right)}\left\|\tilde{\mathbf{x}}\left(t_{0}\right)\right\|+\frac{D}{k}\left(\|\mathbf{a}\| \varepsilon+\|\mathbf{b}\|\left(v_{1}(\varepsilon)+v_{2}\left(\Delta_{\xi}\right)\right)+\Delta_{\xi}\right)$.
Noticing that $z_{1}=\tilde{\mathbf{C}}^{T} \int_{t_{0}}^{t} e^{\Lambda(t-\tau)} \mathbf{G u}(\tau) d \tau$ and denoting $\kappa_{1}(\varepsilon)=2 \frac{D}{k}\left(\|\mathbf{a}\| \varepsilon+\|\mathbf{b}\| v_{1}(\varepsilon)\right)+v_{1}(\varepsilon), \kappa_{2}\left(\Delta_{\xi}\right)=$ $2 \frac{D}{k}\left(\Delta_{\xi}+\|\mathbf{b}\| v_{2}\left(\Delta_{\xi}\right)\right)+v_{2}\left(\Delta_{\xi}\right)$ we can conclude that there is a $t^{\prime}\left(\varepsilon, \mathbf{x}_{0}\right) \geq t_{1}(\varepsilon)$ such that

$$
\left\|z_{1}(\tau)+u_{1}(\tau)\right\|_{\infty,[t, \infty)} \leq \kappa_{1}(\varepsilon)+\kappa_{2}\left(\Delta_{\xi}\right) \forall t \geq t^{\prime}(\varepsilon) .
$$

Noticing that $y(t), \mathbf{d}(t) \equiv 0 \Rightarrow e(t) \equiv 0$ ensures that (66) holds too.

Proof of Lemma 10. Consider $\mathbf{J}(\boldsymbol{\lambda}, t)=\mathbf{z}^{T}\left(\int_{t}^{t+T}\right.$ $\left.\boldsymbol{\alpha}(\tau, \boldsymbol{\lambda}) \boldsymbol{\alpha}^{T}(\tau, \boldsymbol{\lambda}) \mathrm{d} \tau\right) \mathbf{z}=\int_{t}^{t+T}\left\|\mathbf{z}^{T} \boldsymbol{\alpha}(\tau, \boldsymbol{\lambda})\right\|^{2}$, where $\mathbf{z} \in \mathbb{R}^{n+m}, \mathbf{z} \neq 0$, for $t \in \mathbb{R}_{\geq t_{0}}$. According to C2 we have: $\mathbf{J}(\boldsymbol{\lambda}, t) \geq \mu\|\mathbf{z}\|^{2} \forall \boldsymbol{\lambda} \in \Omega_{\lambda}$. Let $\hat{\boldsymbol{\lambda}}: \mathbb{R}_{\geq t_{0}} \rightarrow \Omega_{\lambda}$ be a differentiable function, and consider $\mathbf{J}(\hat{\boldsymbol{\lambda}}(t), t)-$ $\int_{t}^{t+T}\left\|\mathbf{z}^{T} \boldsymbol{\alpha}(\tau, \hat{\boldsymbol{\lambda}}(\tau))\right\|^{2} \mathrm{~d} \tau=\int_{t}^{t+T}\left\|\mathbf{z}^{T} \boldsymbol{\alpha}(\tau, \hat{\boldsymbol{\lambda}}(t))\right\|^{2}$ $-\left\|\mathbf{z}^{T} \boldsymbol{\alpha}(\tau, \hat{\boldsymbol{\lambda}}(\tau))\right\|^{2} \mathrm{~d} \tau=\int_{t}^{t+T}\left\|\mathbf{z}^{T} \boldsymbol{\alpha}(\tau, \hat{\boldsymbol{\lambda}}(t))\right\|^{2}-$ $\mathbf{z}^{T} \boldsymbol{\alpha}(\tau, \hat{\boldsymbol{\lambda}}(t)) \boldsymbol{\alpha}^{T}(\tau, \hat{\boldsymbol{\lambda}}(\tau)) \mathbf{z}+\mathbf{z}^{T} \boldsymbol{\alpha}(\tau, \hat{\boldsymbol{\lambda}}(t)) \boldsymbol{\alpha}^{T}(\tau, \hat{\boldsymbol{\lambda}}(\tau)) \mathbf{z}-$ $\left\|\mathbf{z}^{T} \boldsymbol{\alpha}(\tau, \hat{\boldsymbol{\lambda}}(\tau))\right\|^{2} \mathrm{~d} \tau=\int_{t}^{t+T} \mathbf{z}^{T} \boldsymbol{\alpha}(\tau, \hat{\boldsymbol{\lambda}}(t))\left[\boldsymbol{\alpha}^{T}(\tau, \hat{\boldsymbol{\lambda}}(t))-\right.$ $\left.\boldsymbol{\alpha}^{T}(\tau, \hat{\boldsymbol{\lambda}}(\tau))\right] \mathbf{z}+\int_{t}^{t+T} \mathbf{z}^{T}[\boldsymbol{\alpha}(\tau, \hat{\boldsymbol{\lambda}}(t))-\boldsymbol{\alpha}(\tau, \hat{\boldsymbol{\lambda}}(\tau))]$ $\boldsymbol{\alpha}^{T}(\tau, \hat{\boldsymbol{\lambda}}(\tau)) \mathbf{z d} \tau$. Applying the Cauchy-Schwarz inequality to the last equality, and invoking $\mathrm{C} 4$ and C3 we obtain: $\mathbf{J}(\hat{\boldsymbol{\lambda}}(t), t)-\int_{t}^{t+T}\left\|\mathbf{z}^{T} \boldsymbol{\alpha}(\tau, \hat{\boldsymbol{\lambda}}(\tau))\right\|^{2} \mathrm{~d} \tau \leq$ $\left(\int_{t}^{t+T}\left\|\mathbf{z}^{T}[\boldsymbol{\alpha}(\tau, \hat{\boldsymbol{\lambda}}(t))-\boldsymbol{\alpha}(\tau, \hat{\boldsymbol{\lambda}}(\tau))]\right\|^{2} d \tau\right)^{\frac{1}{2}} 2 M T\|\mathbf{z}\| \leq$ $2 D M T^{2}\|\mathbf{z}\|^{2} \quad \max _{\tau \in[t, t+T]}\|\dot{\hat{\boldsymbol{\lambda}}}(\tau)\|$. Thus (39), (40) ensure that $\int_{t}^{t+T}\left\|\mathbf{z}^{T} \boldsymbol{\alpha}(\tau, \hat{\boldsymbol{\lambda}}(\tau))\right\|^{2} \mathrm{~d} \tau \geq \mathbf{J}(\hat{\boldsymbol{\lambda}}(t), t)-$ $r \mu\|\mathbf{z}\|^{2}$. This, in accordance with $\mathrm{C} 2$, guarantees that $\int_{t}^{t+T}\left\|\mathbf{z}^{T} \boldsymbol{\alpha}(\tau, \hat{\boldsymbol{\lambda}}(\tau))\right\|^{2} \mathrm{~d} \tau \geq(1-r) \mu\|\mathbf{z}\|^{2}$. Hence $\boldsymbol{\alpha}(t, \hat{\boldsymbol{\lambda}}(t))$ is persistently exciting in the sense of Definition 2 . The value of $(1-r) \mu$ does not depend on the choice of $\hat{\boldsymbol{\lambda}}$ as long as (40), (39) hold. Finally, notice that C4 and (39) guarantee boundedness of $\boldsymbol{\alpha}(\cdot, \hat{\boldsymbol{\lambda}}(\cdot))$ and its derivative: $\max \{\|\boldsymbol{\alpha}(t, \hat{\boldsymbol{\lambda}}(t))\|,\|\dot{\boldsymbol{\alpha}}(t, \hat{\boldsymbol{\lambda}}(t))\|\} \leq$ $M+M M_{\lambda}=M+\frac{\mu r}{2 D T^{2}}$. Taking C1 and Theorem 3 into account we conclude that the lemma follows.

Proof of Lemma 11. According to conditions of the lemma, (57), we conclude that $h\left(t_{0}\right) \geq 0$. Introduce a strictly decreasing sequence: $\left\{\sigma_{i}\right\}, i=0,1, \ldots, \sigma_{i}=$ $(1 / \kappa)^{i}, \kappa \in(1, \infty)$. Further, let $\left\{t_{i}\right\}, i=1, \ldots, t_{1}<$ $t_{2}<\cdots<t_{n}<\cdots$ be an ordered infinite sequence:

$$
h\left(t_{i}\right)=\sigma_{i} h\left(t_{0}\right)
$$

If $\left\{t_{i}\right\}$ satisfying (A.1) does not exist then it is clear that $h\left(t_{0}\right) \geq h(t) \geq 0$ for all $t \geq t_{0}$. Hence, in accordance with $(29), \mathbf{x}(\cdot)$ is bounded for all $t \geq t_{0}$, and nothing remains to be proven. Let us now show that if (56), (57), and (A.1) hold then

$$
h(t) \rightarrow 0 \Rightarrow t \rightarrow \infty
$$


Consider $T_{i}=t_{i}-t_{i-1}$. It is clear from (55) that

$$
T_{i} D_{\gamma} \max _{\tau \in\left[t_{i-1}, t_{i}\right]}\|\mathbf{x}(\tau)+\mathbf{d}(\tau)\|_{\varepsilon} \geq h\left(t_{0}\right)\left(\sigma_{i-1}-\sigma_{i}\right) .
$$

In addition, $\max _{\tau \in\left[t_{i-1}, t_{i}\right]}\|\mathbf{x}(\tau)+\mathbf{d}(\tau)\|_{\varepsilon}=\| \mathbf{x}(\tau)+$ $\mathbf{d}(\tau) \|_{\infty,\left[t_{i-1}, t_{i}\right]}-\varepsilon$ if $\|\mathbf{x}(\tau)+\mathbf{d}(\tau)\|_{\infty,\left[t_{i-1}, t_{i}\right]}>\varepsilon$, and $\max _{\tau \in\left[t_{i-1}, t_{i}\right]}\|\mathbf{x}(\tau)+\mathbf{d}(\tau)\|_{\varepsilon}=0$ overwise, we can see from (A.3) that

$$
T_{i} \geq\left\{\begin{array}{c}
\frac{h\left(t_{0}\right)\left(\sigma_{i-1}-\sigma_{i}\right)}{D_{\gamma}} \frac{1}{\|\mathbf{x}(\tau)+\mathbf{d}(\tau)\|_{\infty,\left[t_{i-1}, t_{i}\right]}-\varepsilon} \\
\|\mathbf{x}(\tau)+\mathbf{d}(\tau)\|_{\infty,\left[t_{i-1}, t_{i}\right]}>\varepsilon \\
\infty,\|\mathbf{x}(\tau)+\mathbf{d}(\tau)\|_{\infty,\left[t_{i-1}, t_{i}\right]} \leq \varepsilon
\end{array}\right.
$$

Consider the case when $\|\mathbf{x}(\tau)+\mathbf{d}(\tau)\|_{\infty,\left[t_{i-1}, t_{i}\right]}-\varepsilon>0$ for all $i=1,2, \ldots$. Let us pick

$$
\tau^{*}=\varrho^{-1}(d / \kappa), d \in(0,1),
$$

and select the value of $D_{\gamma}$ such that (57) holds. Given that $\|\mathbf{x}(\tau)+\mathbf{d}(\tau)\|_{\infty,\left[t_{0}, t_{1}\right]}-\varepsilon \leq \varrho(0)\left\|\mathbf{x}\left(t_{0}\right)\right\|+\operatorname{ch}\left(t_{0}\right)+$ $\Delta+\Delta_{d}-\varepsilon$, conditions (57), (56), and (A.5) imply $D_{\gamma} \leq$ $\frac{\kappa-1}{\kappa} \frac{h\left(t_{0}\right)}{\varrho(0)\left\|\mathbf{x}\left(t_{0}\right)\right\|+c \mid h\left(t_{0}\right)} \frac{1}{\tau^{*}} \leq \frac{h\left(t_{0}\right)\left(\sigma_{0}-\sigma_{1}\right)}{\left(\|\mathbf{x}(\tau)+\mathbf{d}(\tau)\|_{\left.\infty,\left[t_{0}, t_{1}\right]-\varepsilon\right)}\right.} \frac{1}{\tau^{*}}$. This, as follows from (A.4), guarantees that $T_{1} \geq \tau^{*}$.

Without loss of generality suppose that there is an $i \geq 2$ : $T_{j} \geq \tau^{*}$ for all $1 \leq j \leq i-1$. Let us show that $T_{i-1} \geq$ $\tau^{*} \Rightarrow T_{i} \geq \tau^{*}$. This will ensure that (A.2) is satisfied and that the lemma hold. Consider $\|\mathbf{x}(\tau)\|_{\infty,\left[t_{i-1}, t_{i}\right]} ;(54)$ and (A.1) imply that: $\|\mathbf{x}(\tau)\|_{\infty,\left[t_{i-1}, t_{i}\right]} \leq \varrho(0)\left\|\mathbf{x}\left(t_{i-1}\right)\right\|+$ $c \sigma_{i-1} h\left(t_{0}\right)+\Delta$. Hence

$$
\begin{aligned}
& \|\mathbf{x}(\tau)\|_{\infty,\left[t_{i-1}, t_{i}\right]} \leq \varrho(0)\left[\varrho\left(T_{i-1}\right)\left\|\mathbf{x}\left(t_{i-2}\right)\right\|+c \sigma_{i-2} h\left(t_{0}\right)\right] \\
& +\varrho(0) \Delta+\operatorname{ch}\left(t_{0}\right) \sigma_{i-1}+\Delta \leq \varrho(0) \varrho\left(\tau^{*}\right)\left\|\mathbf{x}\left(t_{i-2}\right)\right\|+P_{1},
\end{aligned}
$$

where $P_{1}=\varrho(0) c \sigma_{i-2} h\left(t_{0}\right)+c \sigma_{i-1} h\left(t_{0}\right)+\varrho(0) \Delta+\Delta$. Invoking (54) again results in

$$
\|\mathbf{x}(\tau)\|_{\infty,\left[t_{i-1}, t_{i}\right]} \leq \varrho(0) \varrho^{2}\left(\tau^{*}\right)\left\|\mathbf{x}\left(t_{i-3}\right)\right\|+P_{2},
$$

where $P_{2}=\operatorname{ch}\left(t_{0}\right) \varrho(0)\left[\varrho\left(\tau^{*}\right) \sigma_{i-3}+\sigma_{i-2}\right]+c \sigma_{i-1} h\left(t_{0}\right)+$ $\varrho(0)\left[\varrho\left(\tau^{*}\right) \Delta+\Delta\right]+\Delta$, and

$$
\|\mathbf{x}(\tau)\|_{\infty,\left[t_{i-1}, t_{i}\right]} \leq \varrho(0) \varrho^{3}\left(\tau^{*}\right)\left\|\mathbf{x}\left(t_{i-4}\right)\right\|+P_{3},
$$

where $P_{3}=\operatorname{ch}\left(t_{0}\right) \varrho(0)\left[\varrho^{2}\left(\tau^{*}\right) \sigma_{i-4}+\varrho\left(\tau^{*}\right) \sigma_{i-3}+\right.$ $\left.\sigma_{i-2}\right]+c \sigma_{i-1} h\left(t_{0}\right)+\Delta \varrho(0)\left[\varrho\left(\tau^{*}\right)^{2}+\varrho\left(\tau^{*}\right)+1\right]+$ $\Delta=\operatorname{ch}\left(t_{0}\right) \varrho(0)\left[\sum_{j=0}^{2} \varrho^{j}\left(\tau^{*}\right) \sigma_{i-j-2}\right]+\operatorname{ch}\left(t_{0}\right) \sigma_{i-1}+$ $\Delta \varrho(0)\left[\sum_{j=0}^{2} \varrho^{j}\left(\tau^{*}\right)\right]+\Delta$. After $i-1$ steps we obtain

$$
\|\mathbf{x}(\tau)\|_{\infty,\left[t_{i-1}, t_{i}\right]} \leq \varrho(0) \varrho^{i-1}\left(\tau^{*}\right)\left\|\mathbf{x}\left(t_{0}\right)\right\|+P_{i-1},
$$

with $P_{i-1}=\operatorname{ch}\left(t_{0}\right) \varrho(0)\left[\sum_{j=0}^{i-2} \varrho^{j}\left(\tau^{*}\right) \sigma_{i-j-2}\right]+\operatorname{ch}\left(t_{0}\right) \sigma_{i-1}$ $+\Delta \varrho(0)\left[\sum_{j=0}^{i-2} \varrho^{j}\left(\tau^{*}\right)\right]+\Delta$. The values of $T_{i}$, as follows from (A.4), are bounded from below by:

$$
T_{i} \geq \frac{\sigma_{i-1}-\sigma_{i}}{\sigma_{i-1} D_{\gamma} \sigma_{i-1}^{-1}} \frac{h\left(t_{0}\right)}{\left(\|\mathbf{x}(\tau)+\mathbf{d}(\tau)\|_{\infty,\left[t_{i-1}, t_{i}\right]}-\varepsilon\right)}
$$

Consider $\sigma_{i-1}^{-1}\left(\|\mathbf{x}(\tau)+\mathbf{d}(\tau)\|_{\infty,\left[t_{i-1}, t_{i}\right]}-\varepsilon\right)$. Taking (A.6) into account we derive that:

$$
\begin{aligned}
& \sigma_{i-1}^{-1}\left(\|\mathbf{x}(\tau)+\mathbf{d}(\tau)\|_{\infty,\left[t_{i-1}, t_{i}\right]}-\varepsilon\right) \leq \varrho(0) \varrho^{i-1}\left(\tau^{*}\right) \times \\
& \kappa^{i-1}\left\|\mathbf{x}\left(t_{0}\right)\right\|+k^{i-1} P_{i-1}+k^{i-1} \Delta_{d}-k^{i-1} \varepsilon= \\
& \varrho(0) \varrho^{i-1}\left(\tau^{*}\right) \kappa^{i-1}\left\|\mathbf{x}\left(t_{0}\right)\right\|+\operatorname{ch}\left(t_{0}\right) \varrho(0) \kappa\left[\sum_{j=0}^{i-2} \varrho^{j}\left(\tau^{*}\right) \kappa^{j}\right] \\
& +\operatorname{ch}\left(t_{0}\right)+\kappa^{i-1}\left[\Delta\left(\varrho(0) \sum_{j=0}^{i-2} \varrho^{j}\left(\tau^{*}\right)+1\right)+\Delta_{d}-\varepsilon\right] .
\end{aligned}
$$

Noticing that $\tau^{*}$ is chosen in accordance with (A.5) one can therefore obtain:

$$
\begin{aligned}
& \sigma_{i-1}^{-1}\left(\|\mathbf{x}(\tau)+\mathbf{d}(\tau)\|_{\infty,\left[t_{i-1}, t_{i}\right]}-\varepsilon\right) \leq \varrho(0)\left\|\mathbf{x}\left(t_{0}\right)\right\|+ \\
& \operatorname{ch}\left(t_{0}\right)+\operatorname{ch}\left(t_{0}\right) \varrho(0) \kappa \sum_{j=0}^{i-2} d^{j}+ \\
& \kappa^{i-1}\left[\Delta\left(\varrho(0) \sum_{j=0}^{i-2} \frac{d}{\kappa}^{j}+1\right)+\Delta_{d}-\varepsilon\right] \\
& \leq \varrho(0)\left\|\mathbf{x}\left(t_{0}\right)\right\|+\operatorname{ch}\left(t_{0}\right)\left(1+\frac{\varrho(0) \kappa}{1-d}\right)+ \\
& k^{i-1}\left[\Delta\left(\frac{\varrho(0)}{1-d / k}+1\right)+\Delta_{d}-\varepsilon\right] .
\end{aligned}
$$

Condition (56) implies that $\Delta\left(\frac{\varrho(0)}{1-d / k}+1\right)+\Delta_{d}-\varepsilon \leq 0$. Hence $\sigma_{i-1}^{-1}\left(\|\mathbf{x}(\tau)+\mathbf{d}(\tau)\|_{\infty,\left[t_{i-1}, t_{i}\right]}-\varepsilon\right) \leq \varrho(0)\left\|\mathbf{x}\left(t_{0}\right)\right\|+$ $\operatorname{ch}\left(t_{0}\right)\left(1+\frac{\varrho(0) \kappa}{1-d}\right)$. Substituting the latter estimate into (A.7) and using (57) yields $T_{i} \geq \tau^{*}$. Thus $h(\cdot)$ is bounded for $t \geq t_{0}$, and hence so is $\mathbf{x}(\cdot)$.

Proof of Theorem 13. Let $\Lambda_{0}=\mathbf{A}-\mathbf{B C}^{T} \mathbf{A}$, $\mathbf{G}_{0}=\mathbf{I}_{n}-\mathbf{B C}^{T}$. Consider $\tilde{\boldsymbol{\varphi}}\left(t, \hat{\boldsymbol{\lambda}}(t), T_{1}\right)=\mathbf{C}^{T} \mathbf{A} \int_{t-T_{1}}^{t}$ $e^{\Lambda_{0}(t-\tau)} \mathbf{G}_{0} \boldsymbol{\Psi}(\tau, \hat{\boldsymbol{\lambda}}(t), y(\tau)) d \tau+\mathbf{C}^{T} \boldsymbol{\Psi}(t, \hat{\boldsymbol{\lambda}}(t), y(t))$. It is clear that for any $\varepsilon_{1}>0$ there are $T_{1}, t_{1}$ sufficiently large and $\gamma_{1}$ sufficiently small:

$$
\left\|\tilde{\boldsymbol{\varphi}}\left(t, \hat{\boldsymbol{\lambda}}(t), T_{1}\right)-\boldsymbol{\varphi}(t, \hat{\boldsymbol{\lambda}}(t), y(t),[\hat{\boldsymbol{\lambda}}, y])\right\|<\varepsilon_{1}
$$

for all $\gamma \in\left(0, \gamma_{1}\right)$ and $t \geq t_{1} \geq t_{0}$. Indeed, consider $\delta_{1}\left(T_{1}, t\right)=\mathbf{C}^{T} \mathbf{A} e^{\Lambda_{0} T_{1}} \int_{t_{0}}^{t-T_{1}} e^{\Lambda_{0}\left(t-T_{1}-\tau\right)} \mathbf{G}_{0}$ $\boldsymbol{\Psi}(\tau, \hat{\boldsymbol{\lambda}}(\tau), y(\tau)) d \tau, \quad \delta_{2}\left(T_{1}, t\right)=\mathbf{C}^{T} \mathbf{A} \int_{t-T_{1}}^{t} e^{\Lambda_{0}(t-\tau)}$ $\mathbf{G}_{0}(\boldsymbol{\Psi}(\tau, \hat{\boldsymbol{\lambda}}(\tau), y(\tau))-\boldsymbol{\Psi}(\tau, \hat{\boldsymbol{\lambda}}(t), y(\tau))) d \tau$, pick $\varepsilon_{1}>$ 0 , and let $T_{1}$ be so large that $\left|\delta_{1}\left(T_{1}, t\right)\right|<\varepsilon_{1} / 2$ for $t \geq t_{0}+T_{1}$. Let $\gamma_{1} \in \mathbb{R}_{>0}$ be so small that $\left|\delta_{2}\left(T_{1}, t\right)\right|<\varepsilon_{1} / 2$ for all $t \geq t_{0}+T_{1}$ (such a choice is always possible due to that $\boldsymbol{\Psi}$ is Lipschitz in $\hat{\boldsymbol{\lambda}}$ ). Noticing that $\boldsymbol{\varphi}(t, \hat{\boldsymbol{\lambda}}(t), y(t),[\hat{\boldsymbol{\lambda}}, y])=\delta_{1}\left(T_{1}, t\right)+\delta_{2}\left(T_{1}, t\right)+$ $\tilde{\varphi}\left(t, \hat{\boldsymbol{\lambda}}(t), T_{1}\right)$ we can conclude that (A.8) holds.

Given that the restriction of $\boldsymbol{\varphi}(t, \boldsymbol{\lambda}, y(t),[\boldsymbol{\lambda}, y]$ ) (as a function of $t, \boldsymbol{\lambda})$ on $\mathbb{R}_{\geq t_{0}} \times \Omega_{\lambda}$ is $\lambda$-UPE with $T, \mu$, there is a $\varepsilon_{1}$ in (A.8) such that $\tilde{\varphi}\left(t, \lambda, T_{1}\right) \in \lambda \mathrm{UPE}(T, \mu-\epsilon)$, 
$\epsilon \in(0, \mu / 3)$. On the other hand (see the first part of the proof of Lemma 10), there is a $\gamma_{2}$ such that $\tilde{\boldsymbol{\varphi}}\left(t, \hat{\boldsymbol{\lambda}}(t), T_{1}\right)$ is persistently exciting with parameters $T, \mu-2 \epsilon$ for all $\gamma \in\left(0, \gamma_{2}\right)$. Choosing $\gamma \in\left(0, \min \left\{\gamma_{1}, \gamma_{2}\right\}\right)$ and taking (A.8) into account we conclude that the restriction of $\boldsymbol{\varphi}(\cdot, \hat{\boldsymbol{\lambda}}(\cdot), y(\cdot),[\hat{\boldsymbol{\lambda}}, y])$ on $\mathbb{R}_{\geq t_{2}}$ is persistently exciting $\left(t_{2}>t_{1}>t_{0}\right)$ provided that $\varepsilon_{1}$ is small enough and $t_{2}$ is sufficiently large. Thus, invoking the argument presented in Part 2 of the proof of Theorem 7 we can conclude that (61) and (60) hold for the combined system. Convergence of state and parameter estimates can now be shown similarly to the $3 \mathrm{~d}$ part of the proof of Theorem 7 . 